\title{
Striving for cognitive enhancement with RT-FMRI neurofeedback
}

Citation for published version (APA):

Kamp, T. (2018). Striving for cognitive enhancement with RT-FMRI neurofeedback. [Doctoral Thesis, Maastricht University]. Maastricht University. https://doi.org/10.26481/dis.20181130tk

Document status and date:

Published: 01/01/2018

DOI:

10.26481/dis.20181130tk

Document Version:

Publisher's PDF, also known as Version of record

\section{Please check the document version of this publication:}

- A submitted manuscript is the version of the article upon submission and before peer-review. There can be important differences between the submitted version and the official published version of record.

People interested in the research are advised to contact the author for the final version of the publication, or visit the DOI to the publisher's website.

- The final author version and the galley proof are versions of the publication after peer review.

- The final published version features the final layout of the paper including the volume, issue and page numbers.

Link to publication

\footnotetext{
General rights rights.

- You may freely distribute the URL identifying the publication in the public portal. please follow below link for the End User Agreement:

www.umlib.nl/taverne-license

Take down policy

If you believe that this document breaches copyright please contact us at:

repository@maastrichtuniversity.nl

providing details and we will investigate your claim.
}

Copyright and moral rights for the publications made accessible in the public portal are retained by the authors and/or other copyright owners and it is a condition of accessing publications that users recognise and abide by the legal requirements associated with these

- Users may download and print one copy of any publication from the public portal for the purpose of private study or research.

- You may not further distribute the material or use it for any profit-making activity or commercial gain

If the publication is distributed under the terms of Article $25 \mathrm{fa}$ of the Dutch Copyright Act, indicated by the "Taverne" license above, 


\section{STRIVING FOR COGNITIVE ENHANCEMENT WITH RT-FMRI NEUROFEEDBACK}

TABEA KAMP 


\section{(C) Tabea Kamp, Maastricht 2018}

All rights reserved. No part of this dissertation may be reproduced, stored in a retrieval system, or transmitted in any form, or by any means, electronic, mechanical, photocopying, recording or otherwise, without prior written permission from the author.

Printed by: printclub Aachen

Cover and chapter illustrations: Liu zishan/Shutterstock.com ISBN: 978-90-829118-6-2

The work presented in this dissertation was funded by the Human Enhancement and Learning (HEaL) grant provided by Maastricht University. 


\title{
STRIVING FOR COGNITIVE ENHANCEMENT WITH RT-FMRI NEUROFEEDBACK
}

\author{
Dissertation
}

to obtain the degree of Doctor at the Maastricht University, on the authority of the Rector Magnificus Prof.dr. Rianne M. Letschert in accordance with the decision of the Board of Deans, to be defended in public on Friday, 30 ${ }^{\text {th }}$ November 2018 at 10:00 hours by

Tabea Kamp 


\section{SUPERVISOR}

Prof. Dr. Rainer Goebel

\section{CO-SUPERVISOR}

Dr. Bettina Sorger

\section{ASSESSMENT COMMITTEE}

Prof. Dr. David E.J. Linden

Prof. Dr. Alexander Sack (Chair)

Dr. Sepideh Sadaghiani (University of Illinois, USA)

Dr. Frank Scharnowski (University of Zurich, Switzerland) 


\section{TABLE OF CONTENTS}

CHAPTER 1 - GENERAL INTRODUCTION

- 7 -

CHAPTER 2 - REAL-TIME FMRI NEUROFEEDBACK CAN FURTHER

ENHANCE THE ABILITY TO GRADUALLY SELF-REGULATE REGIONAL BRAIN ACTIVATION

CHAPTER 3 - THE PRE-STIMULUS DEFAULT MODE NETWORK STATE PREDICTS COGNITIVE TASK PERFORMANCE LEVELS ON A MENTAL ROTATION TASK

CHAPTER 4 - GRADUAL SELF-REGULATION OF THE DEFAULT MODE NETWORK WITH REAL-TIME FMRI NEUROFEEDBACK

Knowledge Valorisation

Acknowledgements

Curriculum Vitae

Publications 



\section{CHAPTER 1 \\ GENERAL INTRODUCTION}
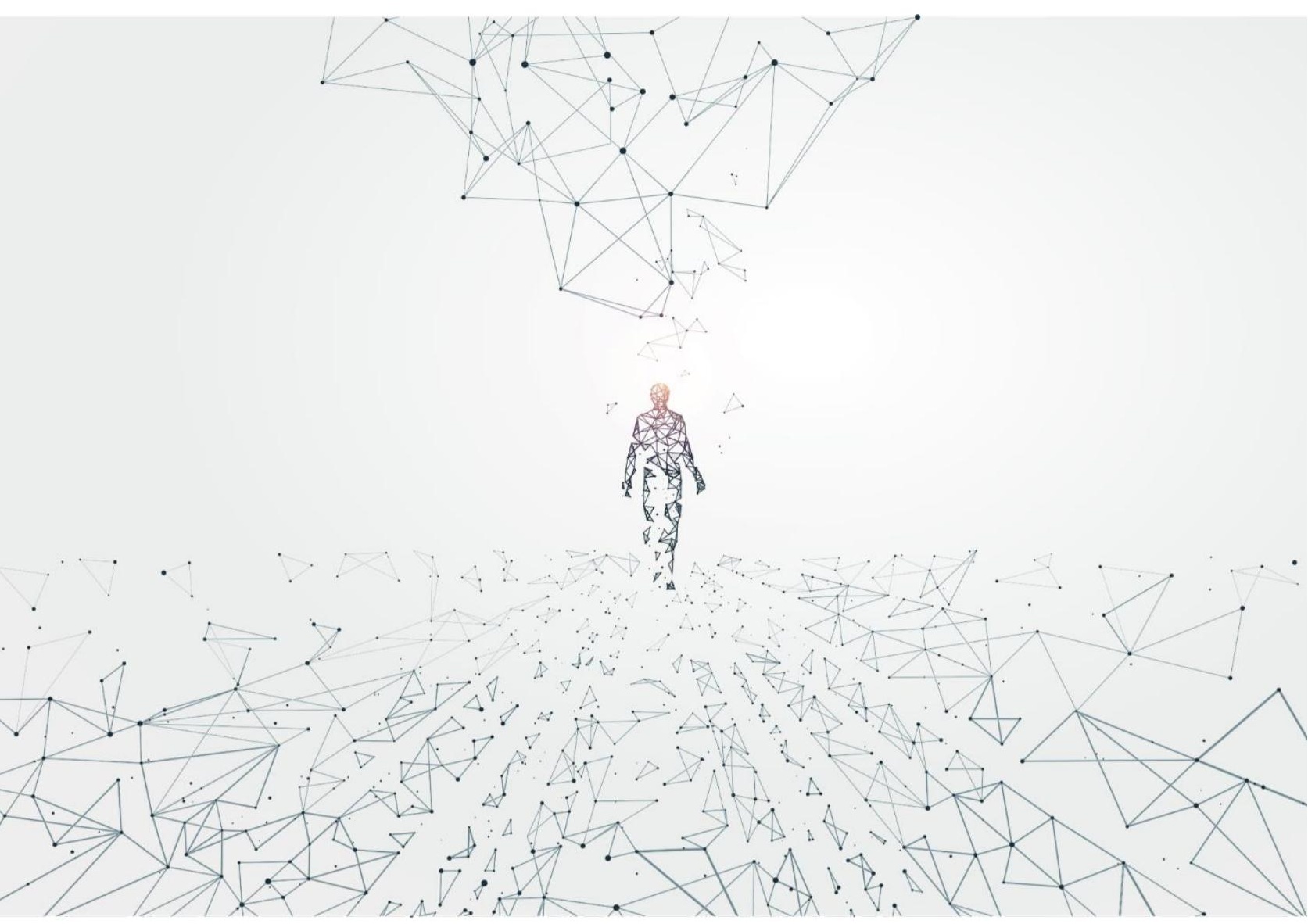

The everlasting strive of humans to modify and enhance their functioning - both physically and mentally - has shaped our society to the highly developed one we live in today. Educational systems have not only developed dramatically over the last centuries, but also became largely available to the general public. At the same time, technological advancements have supported humans in their strive for strengthening their cognitive functions. For example, with the help of external devices and information systems such as computers, the World Wide Web and the smartphone - to name a few important ones - we are capable of accessing and processing information easily and efficiently, and they allow us to reallocate our mental capacities to more complex tasks. While the longing for enhancing cognition is an inherent trait of us humans, the rapidly changing nature of our society also requires us to adapt and act increasingly flexible. While research has mainly focused on restoring cognitive or emotional functions in psychiatric disorders in the past, an increasing amount of studies currently investigates the possibility to go beyond the "baseline" cognitive level and aim at enhancing cognition.

\section{METHODS OF NEURO-ENHANCEMENT TO IMPROVE COGNITION}

In general, conventional strategies for cognitive improvement have been studied quite intensely, demonstrating that several lifestyle aspects influence cognition, such as nutrition, physical exercise, meditative practices, or mnemonics (Dresler et al., 2013). All of these have been shown to be beneficial for cognitive functioning in different ways. However, these life-style choices also require a lot of effort and discipline. Another idea to enhance cognition is to directly modulate neural activation in brain circuits associated with cognitive functions such as memory, attention, processing speed, or executive functions. This neuro-enhancement has been investigated from different angles and with different methodologies, including brain stimulation techniques, psychopharmacological interventions and lately also neurofeedback training (Fig. 1). 


\section{NEURO-ENHANCEMENT}

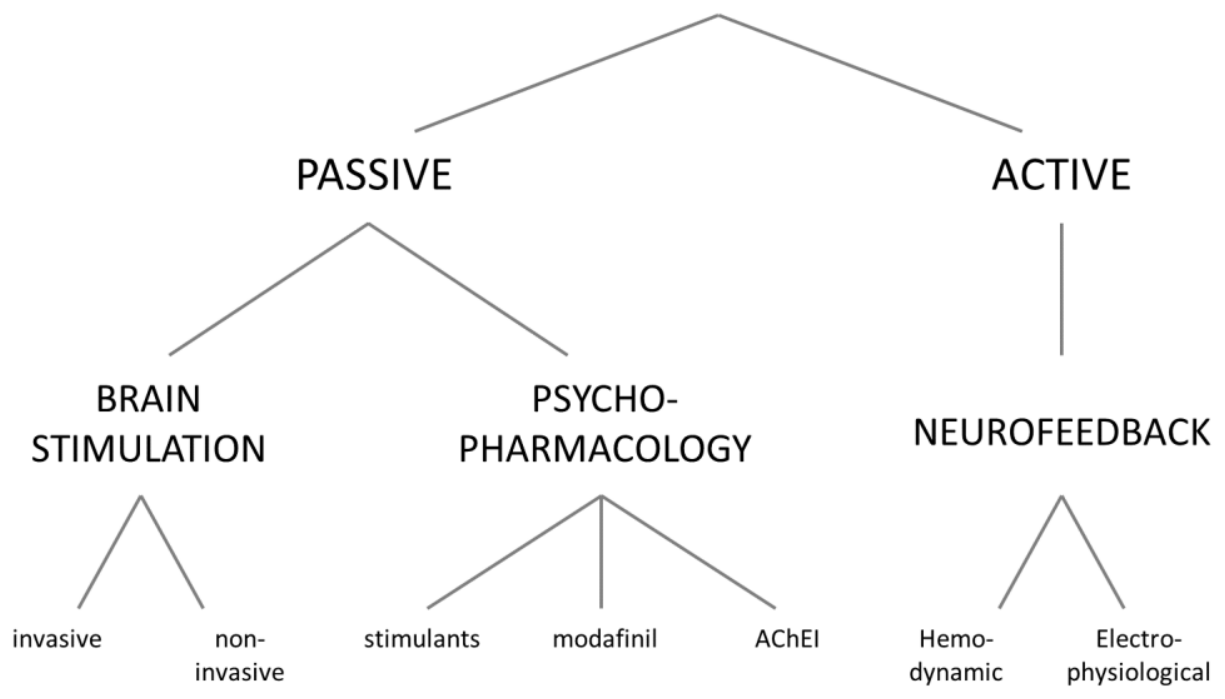

Figure 1: Schematic overview of neuro-enhancement tools to improve cognition.

Abbreviations: AChEl, Acetylcholinesterase inhibitors.

The first branch of methodology involves "passive" neuro-enhancement techniques which modulate cognition externally with either brain stimulation or psychopharmacological interventions, while the latter is also the most controversial branch to improve cognitive functions (Farah, Smith, llieva, \& Hamilton, 2014). There are three classes of medical substances which are investigated as cognitive enhancers: stimulants, modafinil, and acetylcholinesterase inhibitors (Fig. 1). While all of these substances have initially been introduced for the treatment of cognitive impairment in different medical and psychiatric conditions, an increasing amount of research currently investigates their use in neuro-enhancement applications. The first class of drugs includes stimulants such as methylphenidate and amphetamine, which affect the catecholamines dopamine and norepinephrine and are used in the treatment of attention deficit hyperactivity disorder (ADHD) (Hinshaw \& Arnold, 2015). These drugs mostly affect the prefrontal cortex and the regions projecting to it, thus affecting regions that play a major role in $A D H D$ symptomology (Pliszka, 2005). In the context of ADHD as well as cognitive enhancement, the administration of these drugs targets the improvement of 
attention. While it could not be shown consistently that these drugs enhance cognitive functioning beyond a healthy individual level, some evidence exists that methylphenidate improves memory (for a review, see Repantis, Schlattmann, Laisney, \& Heuser, 2010). The second class of neuro-enhancing drugs includes modafinil, a drug which is prescribed in daytime sleepiness associated with narcolepsy to improve wakefulness. However, it could be shown that modafinil has an enhancing effect on attention, even in individuals who were not sleep-deprived (Repantis et al., 2010; Turner et al., 2003). Moreover, it has been used for military personnel in intensely long missions to promote wakefulness (Caldwell \& Caldwell, 2005; Eliyahu, Berlin, Hadad, Heled, \& Moran, 2007). The last class of drugs which has been tested for neuro-enhancing effects on cognition involves acetylcholinesterase inhibitors and memantine, which are prescribed against dementia, including Alzheimer's disease. These drugs aim at improving memory functions, however, no clear effects could be shown so far (Repantis, Laisney, \& Heuser, 2010).

The second "passive" neuro-enhancement methodology involves invasive, such as deep brain stimulation, as well as non-invasive brain stimulation techniques, such as transcranial magnetic stimulation (TMS) and transcranial direct current stimulation (tDCS) (Fig. 1). While deep brain stimulation has only recently been proposed to be used for cognitive enhancement (due to its invasiveness and difficulty to apply) and mixed effects have been reported (Sreekumar, Wittig Jr., Sheehan, \& Zaghloul, 2017), non-invasive approaches have been investigated more extensively. These non-invasive brain stimulation techniques alter brain activation by either interrupting normal brain functioning transiently, or by enhancing it, depending on which stimulation parameters are chosen (Hallett, 2000; Maeda, Keenan, Tormos, Topka, \& Pascual-Leone, 2000; Sack \& Linden, 2003). In TMS, a coil is placed on the head of a participant, which elicits brief magnetic pulses to the scalp. This in turn induces electric currents in the targeted brain tissue which leads to the depolarization of membrane potentials and subsequent neuronal firing. There are different pulse protocols for TMS which result in different behavioural outcomes depending on the pulse frequency and the pulse intensity (Hallett, 2000; Sack \& Linden, 2003). One particular TMS protocol, namely repetitive TMS (rTMS), has been shown to produce behavioural changes which outlast the brain stimulation session consistently (Klomjai, Katz, \& Lackmy-Vallée, 2015; Sack \& 
Linden, 2003). In this protocol, a series of pulses is administered consecutively at a predefined frequency, either at a low frequency $(0.5-2 \mathrm{~Hz})$ for decreasing cortical excitability, or at a high frequency (faster than $5 \mathrm{~Hz}$ ) to induce an increased cortical excitability (Maeda \& Pascual-Leone, 2003). One special and very promising rTMS protocol, namely theta burst TMS, has recently also been reported to produce an enhanced performance on working memory 40min after admission (Hoy et al., 2016). While TMS works on the basis of electromagnetic induction, tDCS directly induces a small electric current (usually $1-2 \mathrm{~mA}$ ) in the targeted brain tissue via two surface electrodes placed on the scalp of the participants (Been, Ngo, Miller, \& Fitzgerald, 2007). Depending on the polarity of the electrode, cortical excitability is either increased (anodal stimulation) or decreased (cathodal stimulation). Like with TMS, it is possible to achieve long-lasting behavioural changes after tDCS stimulation of up to hours (Klomjai et al., 2015). Because it is possible to transiently alter brain activation with TMS and tDCS, causal relationships between brain activation and cognitive functions can be established (Sack \& Linden, 2003). Furthermore, both TMS and tDCS are now increasingly investigated for their potential as neuro-enhancers in order to improve cognitive functions. One review, for example, showed enhanced working memory, learning, and improvements in other executive functions after TMS and tDCS to the left frontal regions (for a review, see Hamilton, Messing, \& Chatterjee, 2011), and additional studies showed an increased mathematical thinking when activating parietal cortex (Cohen Kadosh, Soskic, luculano, Kanai, \& Walsh, 2010; Snowball et al., 2013), as well as increased creativity (Chi \& Snyder, 2012). While TMS and tDCS are both promising tools to enhance cognition, the effects that were shown in small samples need to be extended and replicated. Moreover, there are some side effects associated with these methods. While unlikely, there is a chance of causing a seizure with TMS, alongside chances for headaches and local pain (Taylor, Galvez, \& Loo, 2018), while tDCS can introduce transient feelings of pain, burning or tingling (Kessler, Turkeltaub, Benson, \& Hamilton, 2012).

The abovementioned psychopharmacological and brain stimulation interventions are rather passive methods to induce cognitive enhancement from the receiver's point of view as they do not require any active engagement of the participant except for his/her compliance. Another potential technique to enhance cognition is neurofeedback training. Here, participants are asked to voluntarily self-regulate 
their brain activation with the help of real-time (rt) feedback on this activation, measured with an external neuroimaging device (for a review, see Sulzer et al., 2013). With this training, it is assumed that participants can train their brains to reach specific activation patterns and subsequently access or produce these patterns in a more controlled and volitional fashion. In line with cognitive enhancement, this would mean that participants could learn how to produce brain states which are beneficial for specific cognitive functions and access these deliberately "on demand". This technique, in comparison to psychopharmacology or brain stimulation, thus requires the "active" participation of the subject, as without his/her volitional and deliberate engagement in the task at hand, no explicit training takes place: In a typical neurofeedback experiment, participants try to deliberately modify their brain activation based on initial instructions or naïve strategies and the accompanied presentation of their brain activation, measured continuously by a neuroimaging device, depending on the desired signal to be modulated (Fig. 2). Different neuroimaging methods have been implemented for neurofeedback in the past, the most prominent methods being electroencephalography (EEG) (for a review, see Gruzelier, 2014), magnetoencephalography (MEG) (Florin, Bock, \& Baillet, 2014; Mellinger et al., 2007), as well as hemodynamic measures, such as functional magnetic resonance imaging (fMRI) (for a review, see Thibault, MacPherson, Lifshitz, Roth, \& Raz, 2018) and functional near-infrared spectroscopy (fNIRS) (Lapborisuth, Zhang, Noah, \& Hirsch, 2017; Marx et al., 2015). Each of these methods has advantages and disadvantages, but the most important ones concern spatial and temporal resolution, as well as practicability of the methods: While EEG provides a generally high temporal resolution and therefore picks up fast changes in neural activity, fMRI possesses the advantage of having a high spatial resolution and a high coverage, allowing specific brain regions or networks to be targeted with the neurofeedback training (Poldrack, Mumford, \& Nichols, 2011). This also holds for deeper brain structures, like the amygdala or ventral striatum, which are not accessible with EEG or fNIRS. At the same time, fMRI has a limited temporal resolution, as it relies on the blood oxygenation level dependent (BOLD) response. More specifically, fMRI investigates neuronal activity only indirectly by measuring changes in the local blood flow in the brain, which can be identified based on different magnetic properties of the blood oxygenation (Ogawa, Lee, Kay, \& Tank, 1990). FNIRS 
provides a method which settles in between EEG and fMRI regarding (dis-) advantages, additionally being a portable device. It is another hemodynamic method which assesses the concentration of haemoglobin in the blood by measuring different absorption spectra of oxygenated and deoxygenated haemoglobin in response to near-infrared light induction (Naseer \& Hong, 2015). When comparing fNIRS to EEG and $\mathrm{fMRI}$, it exceeds the spatial resolution of EEG, while being less precise than $\mathrm{fMRI}$. Moreover, although a higher temporal sampling is possible, fNIRS provides a similar temporal resolution as $\mathrm{fMRI}$ because it relies on the hemodynamic response (Strait \& Scheutz, 2014).

While all of the abovementioned methods have shown a potential to enhance cognition, neurofeedback training provides a tool where participants can actively gain access to their own brain activation and cognition and modulate it accordingly, at the same time having little chance for side effects. This - if successful - likely elicits a sense of active agency, self-mastery and achievement in the participant which is rewarding, elicits self-efficacy and self-confidence, and promotes the autonomy of participants. Moreover, rt-fMRI neurofeedback can specifically target individual brain regions for cognitive enhancement, making it possible to directly translate neuroscientific findings about cognitive processes and modulate them in their primary core - the brain. Therefore, rt-fMRI neurofeedback has a great potential to act as a neuro-enhancing tool in order to foster cognition. 

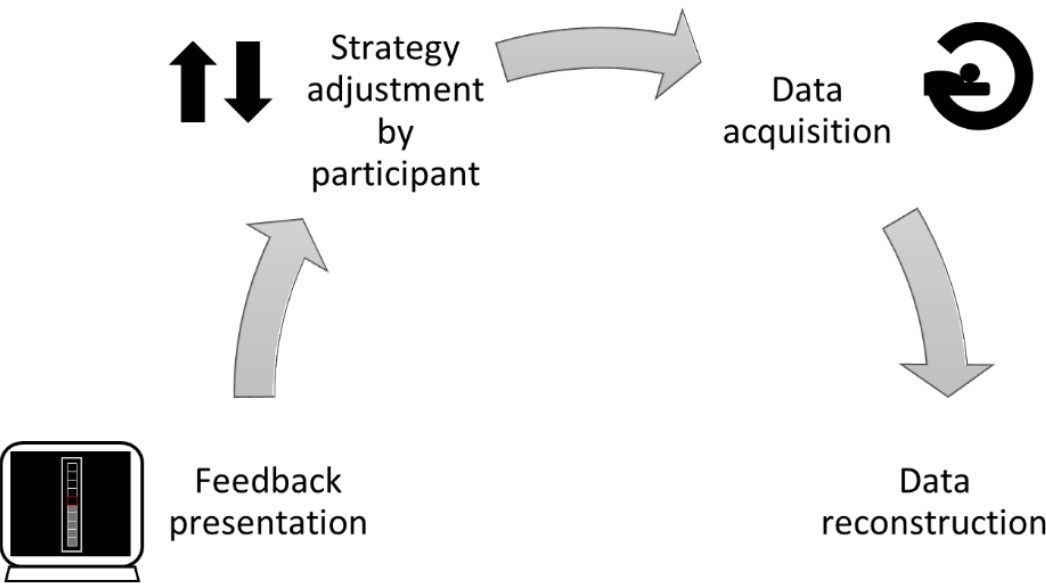

Feedback

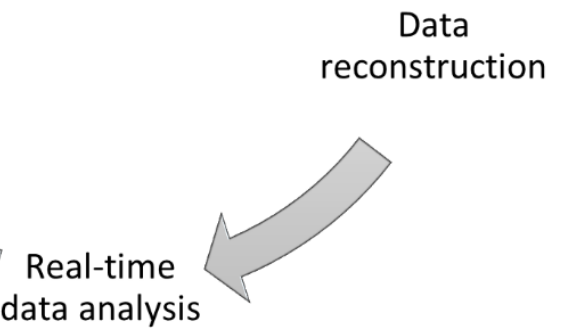

presentation
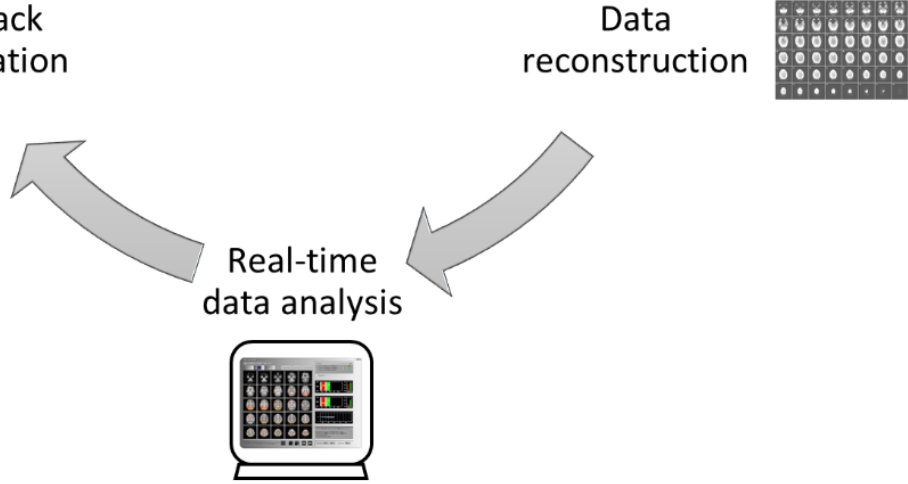

Figure 2: Schematic representation of the rt-fMRI neurofeedback cycle. While lying in the MRI scanner, participants try to self-regulate the targeted brain signal. As soon as volumes are acquired by the scanner, data are reconstructed and analysed in real-time. The interpreted neurofeedback signal is subsequently projected back into the MRI scanner and thus presented to the participant. Based on this neurofeedback information, participants can adjust their strategy how to self-regulate the targeted brain signals.

\section{REAL-TIME FMRI NEUROFEEDBACK AS A NEUROSTIMULATION TOOL TO ENHANCE COGNITION}

Research on rt-fMRI neurofeedback has changed its focus over the years from developing the technological methods and investigating its feasibility to studying its potential application as an add-on to conventional interventions in psychiatric and neurological disorders. With technological advancements in computational power, as well as developments in real-time analysis tools (Goebel \& Linden, 2014; Weiskopf, 2012; Weiskopf et al., 2007) and many feasibility studies, neurofeedback 
has been applied to a number of clinical populations, including patients suffering from depression, schizophrenia, anxiety, tinnitus, obesity, addiction, and ADHD (for a review, see Thibault et al., 2018). Recently, also a convincing double-blind randomised clinical trial has been published investigating neurofeedback effects in depression (Young et al., 2017) and has shown that there is potential in applying this method to clinical populations. However, as a recent comprehensive review examined, rt-fMRI neurofeedback is currently far from being applied consistently in the clinical context. The methodology across existing studies is very variable, there is a lack of proper control groups in the majority of studies, and replications are rare (Thibault et al., 2018).

Next to the application of rt-fMRI neurofeedback to restore cognitive or emotional functions in clinical populations, there has lately been an aim to boost cognition with this technique. More specifically, rt-fMRI neurofeedback has been investigated as a neuro-enhancer in the context of working memory (Sherwood, Kane, Weisend, \& Parker, 2016; Zhang, Yao, Zhang, Long, \& Zhao, 2013), visual performance (Amano, Shibata, Kawato, Sasaki, \& Watanabe, 2016; Shibata, Watanabe, Sasaki, \& Kawato, 2011), and response speed (Yamashita, Hayasaka, Kawato, \& Imamizu, 2017). In this context, it has been tried to boost working memory performance by training the dorsolateral prefrontal cortex (DLPFC) with neurofeedback (Sherwood et al., 2016; Zhang, Yao, et al., 2013). One study showed that an experimental group learned how to self-regulate the DLPFC over time, but also improved on working memory tasks in comparison with a control group that only mentally rehearsed outside of the MRI scanner (Sherwood et al., 2016). Another study also investigated whether the DLPFC could be up-regulated with rt-fMRI and whether this would consecutively lead to increased performance levels in working memory tasks (Zhang, Yao, et al., 2013). The researchers could demonstrate that the experimental group receiving neurofeedback showed a higher activation of the DLPFC after neurofeedback compared to a sham control group. At the same time, the higher DLPFC activation was accompanied with a behavioural advantage of the neurofeedback group on a digit span task. Another stream of research has concentrated on influencing processing in the visual stream with neurofeedback. After neurofeedback training of the activation pattern responsive to a certain line orientation with rt-fMRI neurofeedback, the response to these line orientation stimuli on a visual discrimination task was increased, 
compared to stimuli with different line orientations (Shibata et al., 2011). Moreover, another study tried to induce association learning with rt-fMRI neurofeedback and hereby changed colour perception (Amano et al., 2016). More specifically, participants were presented with a grey-black vertical grating and were asked to increase the size of the grating, which corresponded to the activation pattern associated with the colour red (however unknown to the participants). A subsequent post-test showed that after the neurofeedback training, participants were more likely to "see" or classify an achromatic vertical grating with the colour red than with the colour green. While both studies are not directly linked to cognitive enhancement, they show that it is possible to modify behaviour with the help of neurofeedback.

While all of the abovementioned studies reveal fascinating and promising results, there is also evidence that it is not trivial to show homogenous effects. One study investigated the effects on bi-directional neurofeedback of the connectivity between motor cortex and parietal cortex on cognitive tasks such as vigilance, the Flanker, and the Stroop task (Yamashita et al., 2017). While one group up-regulated the connectivity pattern, the other group down-regulated the same pattern in order to see whether the training would have a diverging effect on cognitive performance. However, while both groups learned to modulate their connectivity in the associated regulation direction, both showed an improvement of reaction times on the Stroop task, and each showed an improvement on one of the other tasks.

One further consideration in using rt-fMRI neurofeedback for cognitive enhancement might be the introduction of a gradual self-regulation approach, in which participants are asked to self-regulate their brain activation to different target levels, rather than maximizing or minimizing their BOLD response as in conventional rt-fMRI neurofeedback paradigms (Chapter 2). On the one hand, this approach allows investigating how well and precise a brain area can be controlled by the participant. On the other hand, and more importantly for cognitive enhancement purposes, such a paradigm could potentially be used in the future as a tool to modify behaviour: in case that different levels of activation in a particular brain area are responsible for different cognitive performance levels, participants could learn to produce the desired activation magnitude in the neurofeedback target region with the help of rt-fMRI neurofeedback. 
The evidence for using rt-fMRI neurofeedback as a tool to enhance cognition is promising, yet limited. Due to this, future research needs to investigate the effects of neurofeedback training on cognitive functions in a structured manner, with careful feasibility studies and subsequent controlled studies.

\section{THE DEFAULT MODE NETWORK, ITS COGNITIVE FUNCTIONS AND ENHACING COGNITION}

Parallel to the development of neurofeedback applications, another topic increasingly became popular in neuroscientific research: the so-called default mode network (DMN) and its role in cognitive functioning. The DMN was formally identified for the first time by showing that across various tasks, certain brain regions are de-activated compared to resting conditions (Buckner, Andrews-Hanna, \& Schacter, 2008; Raichle, 2015). Moreover, these brain regions show a functionally connected and active state when participants are in a "resting state", or "not doing anything in particular". Regions included in this network are the precuneus, the posterior cingulate cortex ( $P C C)$, the medial prefrontal cortex (mPFC), the ventral anterior cingulate cortex (VACC), and parietal regions (Fig.3) (Fox et al., 2005; Fransson, 2005). Throughout the years, the DMN has been implicated in various mental disorders, including Alzheimer's disease, Parkinson's disease, ADHD, schizophrenia, and mood disorders (Broyd et al., 2009; Mohan et al., 2016). These implications and the easiness of data acquisition (i.e., no stimulation or task needed to be performed by the participants) have opened up investigations on the DMN's potential as a biomarker. At the same time, other researchers have focused on the DMN's role in healthy cognitive functioning. In these studies, the DMN has been associated with cognitive processes such as self-referential thoughts and autobiographical memory (Buckner et al., 2008; Spreng \& Grady, 2010), mind-wandering, and daydreaming (Andrews-Hanna, Reidler, Huang, \& Buckner, 2010; Christoff, Gordon, Smallwood, Smith, \& Schooler, 2009; Lieberman, 2007; Mason et al., 2007; Scheibner, Bogler, Gleich, Haynes, \& Bermpohl, 2017; Smallwood \& Schooler, 2015). These mental activities are similar in that the participant is internally-oriented and not concerned with the present external environment. Thus, the DMN may constitute a network which plays a major role in internally-directed cognitive processing and whose activation might not be in favour of high cognitive performance. In fact, the DMN has been associated with 
different states which can be beneficial for or hamper subsequent perceptual and cognitive task performance. More specifically, it has been suggested that the DMN activation prior to a perceptual or cognitive task can predict the performance on the task (Boly et al., 2007; Eichele et al., 2008; Esterman, Noonan, Rosenberg, \& DeGutis, 2013; Mayhew, Ostwald, Porcaro, \& Bagshaw, 2013; Sadaghiani, Hesselmann, \& Kleinschmidt, 2009; Soravia et al., 2016; Weissman, Roberts, Visscher, \& Woldorff, 2006), however resulting in mixed findings across task domains. The DMN thus represents an interesting target for modulation, trying to enhance its functioning in favour of cognitive performance levels. However, its diverse contribution to baseline brain states affecting cognitive performance still requires more research attention in order to identify target DMN baseline states for cognitive enhancement (addressed in Chapter 3).

The spatial resolution of rt-fMRI neurofeedback, as well as the interest in the DMN for diagnostic purposes and its implications in cognitive functioning result in a very promising combination of self-regulating the DMN with rt-fMRI and subsequently investigating its potential for cognitive enhancement. In order to commence such a challenging approach, it first needs to be examined whether humans can learn to self-regulate and thereby volitionally control the DMN with the help of rt-fMRI neurofeedback. One research group has successfully applied rt-fMRI to access and investigate the association between the $\mathrm{DMN}$ and mind-wandering in real time (Garrison, Santoyo, et al., 2013; Garrison, Scheinost, et al., 2013). In their studies, participants could relate a heightened activation of the PCC to their subjective experience of mind-wandering episodes and practiced meditators could also gain control over it. Furthermore, some other studies aimed at self-regulating hubs of the DMN with rt-fMRI neurofeedback (Harmelech, Friedman, \& Malach, 2015; Zhang, Zhang, et al., 2013). Participants in these studies successfully self-regulated the PCC as one of the key hubs of the DMN with the help of neurofeedback, while no study has yet looked at self-regulating several, functionally connected brain regions of the DMN. Moreover, no study so far examined whether it is possible to gradually self-regulate the DMN bi-directionally (by up-and down-regulating it) and thereby accessing finer control mechanisms with neurofeedback. This would additionally enhance participants' feeling of controllability of the DMN, boost their motivation and open up new ways to check for behavioural effects of neurofeedback, as different levels of self-regulation might result in different 
behavioural outcomes, such as variations in reaction times or alterations in task performance levels.

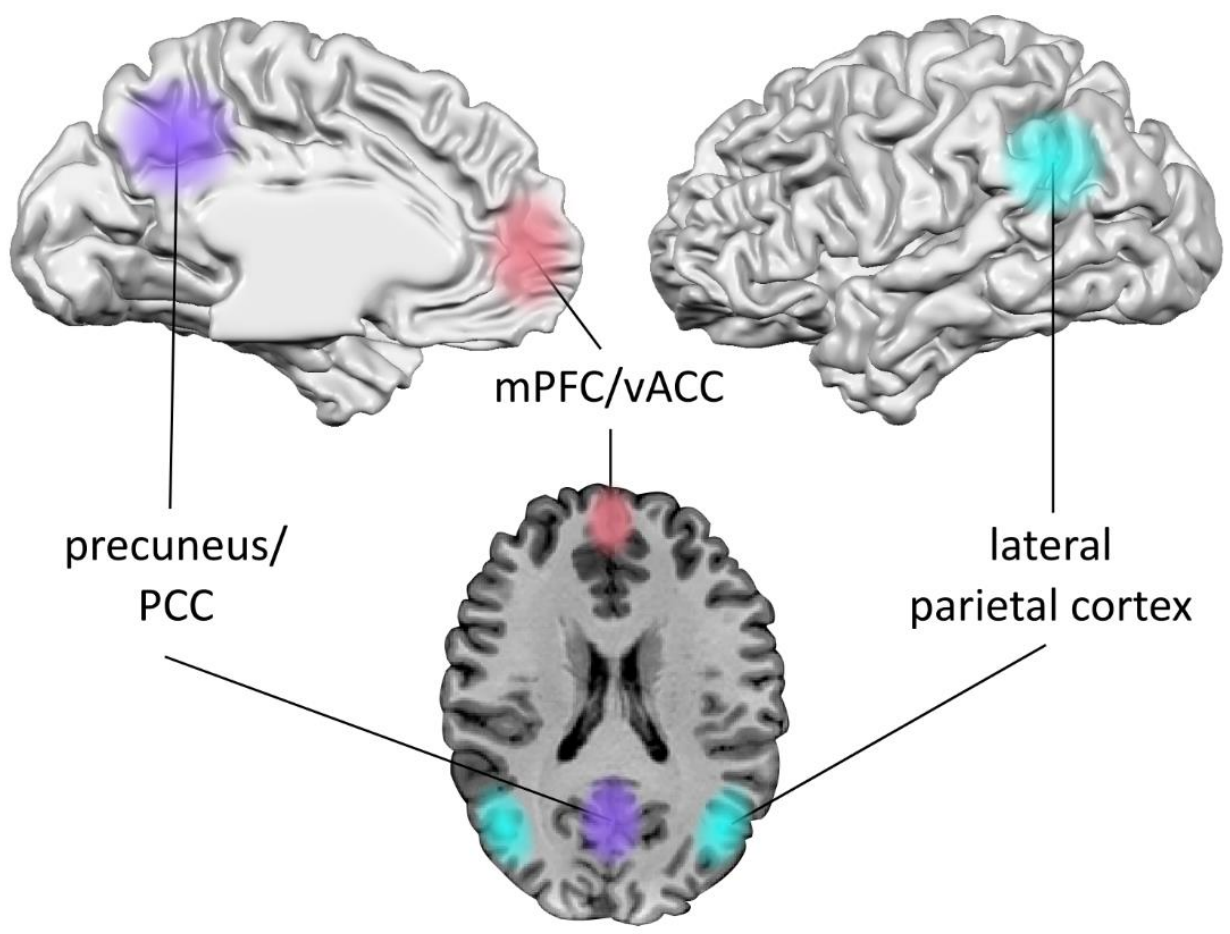

Figure 3: Overview of main hubs of the default mode network.

Abbreviations: PCC, posterior cingulate cortex; MPFC, medial prefrontal cortex; VACC, ventral anterior cingulate cortex.

\section{OUTLINE OF THE DISSERTATION}

In order to strive for cognitive enhancement with rt-fMRI neurofeedback, several elementary steps need to be undertaken, including broadening the spectrum of neurofeedback paradigms tailored for cognitive enhancement, the identification of brain states to be enhanced, and examining whether the desired brain state can be self-regulated with rt-fMRI neurofeedback. The empirical studies presented in this dissertation aimed at establishing evidence for each one of these steps. 
Chapter 2 describes and examines a new neurofeedback paradigm investigating whether humans are able to self-regulate regional brain activation to different predefined target levels. While it was hypothesised that participants would already possess a good capability of self-regulating their brain activation with predefined mental strategies, it was assessed whether rt-fMRI neurofeedback information about their current BOLD activation level could further enhance this capability, also on a single-trial level. This chapter not only proposes a new paradigm to enrich the spectrum of neurofeedback tools, but also provides new means for studying cognitive enhancement with rt-fMRI neurofeedback, as training different levels of brain activation can be used to study potential differences in cognitive performance.

In order to identify potential target brain states to be studied in the context of cognitive enhancement with rt-fMRI neurofeedback, Chapter 3 investigates whether behavioural performance can be predicted on the basis of the pre-stimulus state of the DMN. In this fMRI study, participants performed an extremely sparse event-related mental rotation task and it was assessed whether the correctness and the speed of task performance could be predicted based on the DMN activation and connectivity just prior to task onset.

Finally, Chapter 4 combines the implications from the two previous studies by establishing whether it is feasible to gradually self-regulate the DMN with rt-fMRI neurofeedback in order to achieve a particular, desired brain state. Here, participants underwent two rt-fMRI neurofeedback sessions, one aiming at up-regulating, and one aiming at down-regulating the DMN to three predefined target levels.

The findings of the presented studies are integrated and discussed in Chapter 5, alongside a description of the implications and ethical considerations for cognitive enhancement. 


\section{REFERENCES}

Amano, K., Shibata, K., Kawato, M., Sasaki, Y., \& Watanabe, T. (2016). Learning to Associate Orientation with Color in Early Visual Areas by Associative Decoded fMRI Neurofeedback. Current Biology, 26(14), 1861-1866. http://doi.org/10.1016/j.cub.2016.05.014

Andrews-Hanna, J. R., Reidler, J. S., Huang, C., \& Buckner, R. L. (2010). Evidence for the default network's role in spontaneous cognition. Journal of Neurophysiology, 104(1), 322-35. http://doi.org/10.1152/jn.00830.2009

Been, G., Ngo, T. T., Miller, S. M., \& Fitzgerald, P. B. (2007). The use of tDCS and CVS as methods of non-invasive brain stimulation. Brain Research Reviews, 56(2), 346-361. http://doi.org/10.1016/J.BRAINRESREV.2007.08.001

Boly, M., Balteau, E., Schnakers, C., Degueldre, C., Moonen, G., Luxen, A., ... Laureys, S. (2007). Baseline brain activity fluctuations predict somatosensory perception in humans. Proceedings of the National Academy of Sciences of the United States of America, 104(29), 12187-92. http://doi.org/10.1073/pnas.0611404104

Broyd, S. J., Demanuele, C., Debener, S., Helps, S. K., James, C. J., \& Sonuga-Barke, E. J. S. (2009). Default-mode brain dysfunction in mental disorders: A systematic review. Neuroscience \& Biobehavioral Reviews, 33(3), 279-296. http://doi.org/10.1016/J.NEUBIOREV.2008.09.002

Buckner, R. L., Andrews-Hanna, J. R., \& Schacter, D. L. (2008). The Brain's Default Network: Anatomy, Function, and Relevance to Disease. Annals of the New York Academy of Sciences, 1124(1), 1-38. http://doi.org/10.1196/annals.1440.011

Caldwell, J. A., \& Caldwell, J. L. (2005). Fatigue in military aviation: an overview of US military-approved pharmacological countermeasures. Aviation, Space, and Environmental Medicine, 76(7), C39-51.

Chi, R. P., \& Snyder, A. W. (2012). Brain stimulation enables the solution of an inherently difficult problem. Neuroscience Letters, 515(2), 121-124. http://doi.org/10.1016/J.NEULET.2012.03.012

Christoff, K., Gordon, A. M., Smallwood, J., Smith, R., \& Schooler, J. W. (2009). Experience sampling during fMRI reveals default network and executive system contributions to mind wandering. Proceedings of the National 
Academy of Sciences of the United States of America, 106(21), 8719-24. http://doi.org/10.1073/pnas.0900234106

Cohen Kadosh, R., Soskic, S., luculano, T., Kanai, R., \& Walsh, V. (2010). Modulating neuronal activity produces specific and long-lasting changes in numerical competence. Current Biology, 20(22), 2016-20. http://doi.org/10.1016/j.cub.2010.10.007

Dresler, M., Sandberg, A., Ohla, K., Bublitz, C., Trenado, C., Mroczko-Wąsowicz, A., ... Repantis, D. (2013). Non-pharmacological cognitive enhancement. Neuropharmacology, 64, 529-543. http://doi.org/10.1016/J.NEUROPHARM.2012.07.002

Eichele, T., Debener, S., Calhoun, V. D., Specht, K., Engel, A. K., Hugdahl, K., ... Ullsperger, M. (2008). Prediction of human errors by maladaptive changes in event-related brain networks. Proceedings of the National Academy of Sciences of the United States of America, 105(16), 6173-8. http://doi.org/10.1073/pnas.0708965105

Eliyahu, U., Berlin, S., Hadad, E., Heled, Y., \& Moran, D. S. (2007). Psychostimulants and military operations. Military Medicine, 172(4), 383-7.

Esterman, M., Noonan, S. K., Rosenberg, M., \& DeGutis, J. (2013). In the Zone or Zoning Out? Tracking Behavioral and Neural Fluctuations During Sustained Attention. Cerebral Cortex, 23(11), 2712-2723. http://doi.org/10.1093/cercor/bhs261

Farah, M. J., Smith, M. E., Ilieva, I., \& Hamilton, R. H. (2014). Cognitive enhancement. Wiley Interdisciplinary Reviews: Cognitive Science, 5, 95103. http://doi.org/10.1002/wcs.1250

Florin, E., Bock, E., \& Baillet, S. (2014). Targeted reinforcement of neural oscillatory activity with real-time neuroimaging feedback. Neurolmage, 88, 54-60. http://doi.org/10.1016/J.NEUROIMAGE.2013.10.028

Fox, M. D., Snyder, A. Z., Vincent, J. L., Corbetta, M., Van Essen, D. C., \& Raichle, M. E. (2005). The human brain is intrinsically organised into dynamic, anticorrelated functional networks. Proceedings of the National Academy of Sciences of the United States of America, 102(27), 9673-8. http://doi.org/10.1073/pnas.0504136102 
Fransson, P. (2005). Spontaneous low-frequency BOLD signal fluctuations: An fMRI investigation of the resting-state default mode of brain function hypothesis. Human Brain Mapping, 26(1), 15-29. http://doi.org/10.1002/hbm.20113

Garrison, K. A., Santoyo, J. F., Davis, J. H., Thornhill, T. A., Kerr, C. E., \& Brewer, J. A. (2013). Effortless awareness: using real time neurofeedback to investigate correlates of posterior cingulate cortex activity in meditators' self-report. Frontiers in Human Neuroscience, 7, 440. http://doi.org/10.3389/fnhum.2013.00440

Garrison, K. A., Scheinost, D., Worhunsky, P. D., Elwafi, H. M., Thornhill, T. A., Thompson, E., ... Brewer, J. A. (2013). Real-time fMRI links subjective experience with brain activity during focused attention. Neurolmage, 81 , 110-8. http://doi.org/10.1016/j.neuroimage.2013.05.030

Goebel, R., \& Linden, D. E. J. (2014). Neurofeedback with Real-Time Functional MRI. In C. Mulert \& M. E. Shenton (Eds.), MRI in Psychiatry (pp. 35-46). Berlin, Heidelberg: Springer Berlin Heidelberg. http://doi.org/10.1007/978-3-64254542-9_2

Gruzelier, J. H. (2014). EEG-neurofeedback for optimising performance. I: A review of cognitive and affective outcome in healthy participants. Neuroscience $\&$ Biobehavioral Reviews, 44, http://doi.org/10.1016/J.NEUBIOREV.2013.09.015

Hallett, M. (2000). Transcranial magnetic stimulation and the human brain. Nature, 406(6792), 147-150. http://doi.org/10.1038/35018000

Hamilton, R., Messing, S., \& Chatterjee, A. (2011). Rethinking the thinking cap: ethics of neural enhancement using noninvasive brain stimulation. Neurology, 76(2), 187-93. http://doi.org/10.1212/WNL.0b013e318205d50d

Harmelech, T., Friedman, D., \& Malach, R. (2015). Differential magnetic resonance neurofeedback modulations across extrinsic (visual) and intrinsic (defaultmode) nodes of the human cortex. The Journal of Neuroscience, 35(6), 2588-95. http://doi.org/10.1523/JNEUROSCI.3098-14.2015

Hinshaw, S. P., \& Arnold, L. E. (2015). Attention-deficit hyperactivity disorder, multimodal treatment, and longitudinal outcome: evidence, paradox, and 
challenge. Wiley Interdisciplinary Reviews: Cognitive Science, 6(1), 39-52. http://doi.org/10.1002/wcs.1324

Hoy, K. E., Bailey, N., Michael, M., Fitzgibbon, B., Rogasch, N. C., Saeki, T., \& Fitzgerald, P. B. (2016). Enhancement of Working Memory and TaskRelated Oscillatory Activity Following Intermittent Theta Burst Stimulation in Healthy Controls. Cerebral Cortex, 26(12), 4563-4573. http://doi.org/10.1093/cercor/bhv193

Kessler, S. K., Turkeltaub, P. E., Benson, J. G., \& Hamilton, R. H. (2012). Differences in the experience of active and sham transcranial direct current stimulation. Brain Stimulation, 5(2), 155-62. http://doi.org/10.1016/j.brs.2011.02.007

Klomjai, W., Katz, R., \& Lackmy-Vallée, A. (2015). Basic principles of transcranial magnetic stimulation (TMS) and repetitive TMS (rTMS). Annals of Physical and Rehabilitation Medicine, 58(4), 208-213. http://doi.org/10.1016/J.REHAB.2015.05.005

Lapborisuth, P., Zhang, X., Noah, A., \& Hirsch, J. (2017). Neurofeedback-based functional near-infrared spectroscopy upregulates motor cortex activity in imagined motor tasks. Neurophotonics, 4(2), 021107. http://doi.org/10.1117/1.NPh.4.2.021107

Lieberman, M. D. (2007). Social Cognitive Neuroscience: A Review of Core Processes. Annual Review of Psychology, 58(1), 259-289. http://doi.org/10.1146/annurev.psych.58.110405.085654

Maeda, F., Keenan, J. P., Tormos, J. M., Topka, H., \& Pascual-Leone, A. (2000). Modulation of corticospinal excitability by repetitive transcranial magnetic stimulation. Clinical Neurophysiology, 111(5), 800-5. http://doi.org/10.1016/S1388-2457(99)00323-5

Maeda, F., \& Pascual-Leone, A. (2003). Transcranial magnetic stimulation: studying motor neurophysiology of psychiatric disorders. Psychopharmacology, 168(4), 359-376. http://doi.org/10.1007/s00213-002-1216-x

Marx, A.-M., Ehlis, A.-C., Furdea, A., Holtmann, M., Banaschewski, T., Brandeis, D., ... Strehl, U. (2015). Near-infrared spectroscopy (NIRS) neurofeedback as a treatment for children with attention deficit hyperactivity disorder (ADHD) 
- a pilot study. Frontiers in Human Neuroscience, 8, 1038. http://doi.org/10.3389/fnhum.2014.01038

Mason, M. F., Norton, M. I., Van Horn, J. D., Wegner, D. M., Grafton, S. T., \& Macrae, C. N. (2007). Wandering minds: the default network and stimulusindependent thought. Science, 315(5810), 393-5. http://doi.org/10.1126/science.1131295

Mayhew, S. D., Ostwald, D., Porcaro, C., \& Bagshaw, A. P. (2013). Spontaneous EEG alpha oscillation interacts with positive and negative BOLD responses in the visual-auditory cortices and default-mode network. Neurolmage, 76, 362372. http://doi.org/10.1016/J.NEUROIMAGE.2013.02.070

Mellinger, J., Schalk, G., Braun, C., Preissl, H., Rosenstiel, W., Birbaumer, N., \& Kübler, A. (2007). An MEG-based Brain-Computer Interface (BCl). Neurolmage, 36(3), 581-593. http://doi.org/10.1016/j.micinf.2011.07.011.Innate

Mohan, A., Roberto, A. J., Mohan, A., Lorenzo, A., Jones, K., Carney, M. J., ... Lapidus, K. A. B. (2016). The Significance of the Default Mode Network (DMN) in Neurological and Neuropsychiatric Disorders: A Review. The Yale Journal of Biology and Medicine, 89(1), 49-57.

Naseer, N., \& Hong, K.-S. (2015). fNIRS-based brain-computer interfaces: a review. Frontiers in Human Neuroscience, 9(3), 1-15. http://doi.org/10.3389/fnhum.2015.00003

Ogawa, S., Lee, T. M., Kay, A. R., \& Tank, D. W. (1990). Brain magnetic resonance imaging with contrast dependent on blood oxygenation. Proceedings of the National Academy of Sciences of the United States of America, 87(24), 9868-72.

Pliszka, S. R. (2005). The Neuropsychopharmacology of AttentionDeficit/Hyperactivity Disorder. Biological Psychiatry, 57(11), 1385-1390. http://doi.org/10.1016/j.biopsych.2004.08.026

Poldrack, R., Mumford, J., \& Nichols, T. (2011). Handbook of functional MRI data analysis. Cambridge University Press.

Raichle, M. E. (2015). The Brain's Default Mode Network. Annual Review of Neuroscience, 38, 433-447. http://doi.org/10.1146/annurev-neuro071013-014030 
Repantis, D., Laisney, O., \& Heuser, I. (2010). Acetylcholinesterase inhibitors and memantine for neuroenhancement in healthy individuals: A systematic review. Pharmacological Research, 61(6), 473-481. http://doi.org/10.1016/J.PHRS.2010.02.009

Repantis, D., Schlattmann, P., Laisney, O., \& Heuser, I. (2010). Modafinil and methylphenidate for neuroenhancement in healthy individuals: A systematic review. Pharmacological Research, 62(3), 187-206. http://doi.org/10.1016/J.PHRS.2010.04.002

Sack, A. T., \& Linden, D. E. J. (2003). Combining transcranial magnetic stimulation and functional imaging in cognitive brain research: possibilities and limitations. Brain Research Reviews, 43(1), 41-56. http://doi.org/10.1016/S0165-0173(03)00191-7

Sadaghiani, S., Hesselmann, G., \& Kleinschmidt, A. (2009). Distributed and antagonistic contributions of ongoing activity fluctuations to auditory stimulus detection. The Journal of Neuroscience, 29(42), 13410-7. http://doi.org/10.1523/JNEUROSCI.2592-09.2009

Scheibner, H. J., Bogler, C., Gleich, T., Haynes, J.-D., \& Bermpohl, F. (2017). Internal and external attention and the default mode network. Neurolmage, 148, 381-389. http://doi.org/10.1016/J.NEUROIMAGE.2017.01.044

Sherwood, M. S., Kane, J. H., Weisend, M. P., \& Parker, J. G. (2016). Enhanced control of dorsolateral prefrontal cortex neurophysiology with real-time functional magnetic resonance imaging (rt-fMRI) neurofeedback training and working memory practice. Neurolmage, 124, 214-223. http://doi.org/10.1016/J.NEUROIMAGE.2015.08.074

Shibata, K., Watanabe, T., Sasaki, Y., \& Kawato, M. (2011). Perceptual learning incepted by decoded fMRI neurofeedback without stimulus presentation. Science, 334(6061), 1413-5. http://doi.org/10.1126/science.1212003

Smallwood, J., \& Schooler, J. W. (2015). The Science of Mind Wandering: Empirically Navigating the Stream of Consciousness. Annual Review of Psychology, 66(1), 487-518. http://doi.org/10.1146/annurev-psych-010814-015331

Snowball, A., Tachtsidis, I., Popescu, T., Thompson, J., Delazer, M., Zamarian, L., ... Cohen Kadosh, R. (2013). Long-term enhancement of brain function and 
cognition using cognitive training and brain stimulation. Current Biology, 23(11), 987-92. http://doi.org/10.1016/j.cub.2013.04.045

Soravia, L. M., Witmer, J. S., Schwab, S., Nakataki, M., Dierks, T., Wiest, R., ... Jann, K. (2016). Prestimulus default mode activity influences depth of processing and recognition in an emotional memory task. Human Brain Mapping, 37(3), 924-932. http://doi.org/10.1002/hbm.23076

Spreng, R. N., \& Grady, C. L. (2010). Patterns of Brain Activity Supporting Autobiographical Memory, Prospection, and Theory of Mind, and Their Relationship to the Default Mode Network. Journal of Cognitive Neuroscience, 22(6), 1112-1123. http://doi.org/10.1162/jocn.2009.21282

Sreekumar, V., Wittig Jr., J. H., Sheehan, T. C., \& Zaghloul, K. A. (2017). Principled Approaches to Direct Brain Stimulation for Cognitive Enhancement. Frontiers in Neuroscience, 11, http://doi.org/10.3389/fnins.2017.00650

Strait, M., \& Scheutz, M. (2014). What we can and cannot (yet) do with functional near infrared spectroscopy. Frontiers in Neuroscience, 8(117), 1-12. http://doi.org/10.3389/fnins.2014.00117

Sulzer, J., Haller, S., Scharnowski, F., Weiskopf, N., Birbaumer, N., Blefari, M. L., ... Sitaram, R. (2013). Real-time fMRI neurofeedback: Progress and challenges. Neurolmage, 76, 386-399. http://doi.org/10.1016/J.NEUROIMAGE.2013.03.033

Taylor, R., Galvez, V., \& Loo, C. (2018). Transcranial magnetic stimulation (TMS) safety: a practical guide for psychiatrists. Australasian Psychiatry, 26(2), 189-192. http://doi.org/10.1177/1039856217748249

Thibault, R. T., MacPherson, A., Lifshitz, M., Roth, R. R., \& Raz, A. (2018). Neurofeedback with fMRI: A critical systematic review. Neurolmage, 172, 786-807. http://doi.org/10.1016/J.NEUROIMAGE.2017.12.071

Turner, D. C., Robbins, T. W., Clark, L., Aron, A. R., Dowson, J., \& Sahakian, B. J. (2003). Cognitive enhancing effects of modafinil in healthy volunteers. Psychopharmacology, 165(3), 260-269. http://doi.org/10.1007/s00213002-1250-8 
Weiskopf, N. (2012). Real-time fMRI and its application to neurofeedback. Neurolmage, 62(2), 682-92. http://doi.org/10.1016/j.neuroimage.2011.10.009

Weiskopf, N., Sitaram, R., Josephs, O., Veit, R., Scharnowski, F., Goebel, R., ... Mathiak, K. (2007). Real-time functional magnetic resonance imaging: methods and applications. Magnetic Resonance Imaging, 25(6), 989-1003. http://doi.org/10.1016/J.MRI.2007.02.007

Weissman, D. H., Roberts, K. C., Visscher, K. M., \& Woldorff, M. G. (2006). The neural bases of momentary lapses in attention. Nature Neuroscience, 9(7), 971-978. http://doi.org/10.1038/nn1727

Yamashita, A., Hayasaka, S., Kawato, M., \& Imamizu, H. (2017). Connectivity Neurofeedback Training Can Differentially Change Functional Connectivity and Cognitive Performance. Cerebral Cortex, 27(10), 4960-4970. http://doi.org/10.1093/cercor/bhx177

Young, K. D., Siegle, G. J., Zotev, V., Phillips, R., Misaki, M., Yuan, H., ... Bodurka, J. (2017). Randomized Clinical Trial of Real-Time fMRI Amygdala Neurofeedback for Major Depressive Disorder: Effects on Symptoms and Autobiographical Memory Recall. American Journal of Psychiatry, 174(8), 748-755. http://doi.org/10.1176/appi.ajp.2017.16060637

Zhang, G., Yao, L., Zhang, H., Long, Z., \& Zhao, X. (2013). Improved working memory performance through self-regulation of dorsal lateral prefrontal cortex activation using real-time fMRI. PloS One, 8(8), e73735. http://doi.org/10.1371/journal.pone.0073735

Zhang, G., Zhang, H., Li, X., Zhao, X., Yao, L., \& Long, Z. (2013). Functional Alteration of the DMN by Learned Regulation of the PCC Using Real-Time fMRI. IEEE Transactions on Neural Systems and Rehabilitation Engineering, 21(4), 595-606. http://doi.org/10.1109/TNSRE.2012.2221480 

CHAPTER 2

REAL-TIME FMRI NEUROFEEDBACK CAN FURTHER ENHANCE THE ABILITY TO GRADUALLY SELF-REGULATE REGIONAL BRAIN ACTIVATION
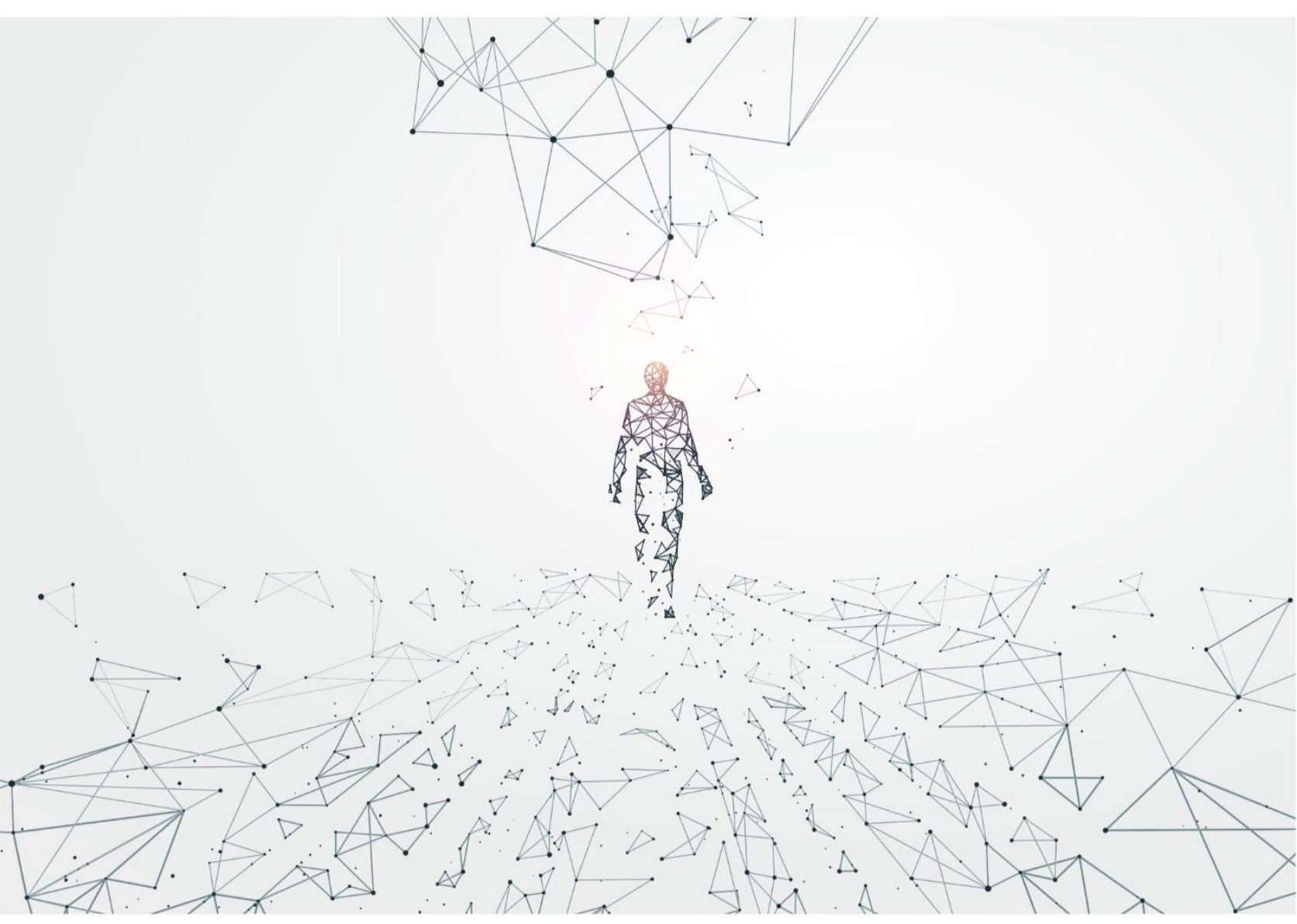


\section{Based on:}

Sorger, B.*, Kamp, T.*, Weiskopf, N., Peters, J. C., \& Goebel, R. (2018). When the brain takes 'BOLD' steps: Real-time fMRI neurofeedback can further enhance the ability to gradually self-regulate regional brain activation. Neuroscience, 378, 71-88. DOI: 10.1016/j.neuroscience.2016.09.026

*Authors contributed equally and share first-authorship. 


\section{ABSTRACT}

Brain-computer interfaces ( $\mathrm{BCls}$ ) based on real-time functional magnetic resonance imaging (rt-fMRI) are currently explored in the context of developing alternative (motor-independent) communication and control means for the severely disabled. In such $\mathrm{BCl}$ systems, the user encodes a particular intention (e.g., an answer to a question or an intended action) by evoking specific mental activity resulting in a distinct brain state that can be decoded from fMRI activation. One goal in this context is to increase the degrees of freedom in encoding different intentions, i.e., to allow the $\mathrm{BCl}$ user to choose from as many options as possible. Recently, the ability to voluntarily modulate spatial and/or temporal blood oxygenation level-dependent (BOLD) -signal features have been explored implementing different mental tasks and/or different encoding time intervals, respectively.

Our two-session fMRI feasibility study systematically investigated for the first time the possibility of using magnitudinal BOLD-signal features for intention encoding. Particularly, in our novel paradigm, participants $(n=10)$ were asked to alternately self-regulate their regional brain-activation level to $30 \%, 60 \%$ or $90 \%$ of their maximal capacity by applying a selected activation strategy (i.e., performing a mental task, e.g., inner speech) and modulation strategies (e.g., using different speech rates) suggested by the experimenters. In a second step, we tested the hypothesis that the additional availability of feedback information on the current BOLD-signal level within a region of interest improves the gradual self-regulation performance. Therefore, participants were provided with neurofeedback in one of the two fMRI sessions. Our results show that the majority of the participants were able to gradually self-regulate regional brain activation to at least two target levels even in the absence of neurofeedback. When provided with continuous feedback on their current BOLD-signal level, most participants further enhanced their gradual self-regulation ability.

Our findings were observed across a wide variety of mental tasks and across clinical MR field strengths (i.e., at 1.5T and 3T), indicating that these findings are robust and can be generalised across mental tasks and scanner types. The suggested novel parametric activation paradigm enriches the spectrum of current rt-fMRI neurofeedback and $\mathrm{BCl}$ methodology and has considerable potential for fundamental and clinical neuroscience applications. 


\section{INTRODUCTION}

Real-time functional magnetic resonance imaging (rt-fMRI) allows for brain-computer interfacing - therewith providing a tool to monitor and alter current a) brain activation (both regionally [e.g., Caria et al., 2007; Zotev et al., 2011; Linden et al., 2012; Canterberry et al., 2013; Greer, Trujillo, Glover, \& Knutson et al., 2014; Young, Zotev, et al., 2014; Cordes et al., 2015] and in widely distributed regions [e.g., LaConte, Peltier, \& Hu, 2007]) or b) brain connectivity patterns (e.g., Koush et al., 2013; Kim, Yoo, Tegethoff, Meinlschmidt, \& Lee, 2015). Rt-fMRI research focuses on two application possibilities of brain-computer interfaces (BCls): neurofeedback learning/therapy and brain-based communication and control (Goebel, Zilverstand, \& Sorger, 2010).

Since the introduction of the rt-fMRI method in 1995 (Cox, Jesmanowicz, \& Hyde, 1995), numerous studies have investigated its suitability for neurofeedback applications. In several proof-of-principle studies with healthy participants, it has been shown that different kinds of overt (behavioural) and covert (mental) tasks can be used to voluntarily in- or decrease (up- or downregulate) the blood oxygenation level-dependent (BOLD) signal in various cortical brain regions, including sensory (e.g., Haller, Birbaumer, \& Veit, 2010; Scharnowski, Hutton, Josephs, Weiskopf, \& Rees , 2012; Robineau et al., 2014; Auer, Schweizer, \& Frahm, 2015), (pre)motor (e.g., Yoo \& Jolesz, 2002; DeCharms et al., 2004; Berman, Horovitz, Venkataraman, \& Hallett, 2012; Chiew, LaConte, \& Graham, 2012), insular (e.g., Caria et al., 2007; Berman, Horovitz, \& Hallett, 2013; Ruiz, Lee, et al., 2013; Emmert et al., 2014), anterior cingulate (e.g., DeCharms et al., 2005; Canterberry et al., 2013; Emmert et al., 2014; Guan et al., 2015), posterior cingulate (Garrison, Santoya, et al., 2013, Garrison, Scheinost, et al., 2013; Zhang, Zhang, et al., 2013), dorsolateral prefrontal (Zhang, Yao, Zhang, Long, \& Zhao, 2013; Sherwood, Kane, Weisend, \& Parker, 2016), inferior frontal (Rota et al., 2009) and orbitofrontal cortex (Hampson et al., 2012; Scheinost et al., 2013), as well as subcortical structures, including nucleus accumbens (Greer et al., 2014), amygdala (e.g., Zotev et al., 2011; Brühl et al., 2014; Young, Zotev, et al., 2014), striatum (Kirsch, Gruber, Ruf, Kiefer, \& Kirsch, 2015), substantia nigra (Sulzer, Sitaram, et al., 2013), and ventral tegmental area (Maclnnes, Dickerson, Chen, \& Adcock, 2016). As an extension, translational studies explored the feasibility of rt-fMRI neurofeedback to remediate pathological brain activation associated with symptoms of various 
(mostly neurological and psychiatric) disorders including major depressive disorder (Linden et al., 2012; Young, Zotev, et al., 2014; Hamilton et al., 2016; Zotev et al., 2016), schizophrenia (Ruiz, Birbaumer, \& Sitaram, 2013; Cordes et al., 2015), Parkinson's disease (Subramanian et al., 2011), spider phobia (Zilverstand, Sorger, Sarkheil, \& Goebel., 2015), chronic pain (DeCharms et al., 2005; Guan et al., 2015), tinnitus (Haller et al., 2010), addiction (Canterberry et al., 2013; Li et al., 2013; Karch et al., 2015; Kirsch et al., 2015; Hartwell et al., 2016), obesity (Frank et al., 2012), autism (Caria \& de Falco, 2015), and stroke (Chiew et al., 2012; Young, Nigogosyan et al., 2014).

The second application possibility of rt-fMRI, the employment of $\mathrm{BCls}$ for motor-independent communication and control, also has considerable societal impact - being potentially of great importance for the severely disabled (e.g., 'locked-in' syndrome [LIS] patients). For almost 30 years now, most BCl researchers have focused on developing communication and control $\mathrm{BCls}$ based on neuroelectric signals (Farwell \& Donchin, 1988; Chapin, Moxon, Markowitz, \& Nicolelis, 1999; Leuthardt, Schalk, Wolpaw, Ojemann, \& Moran, 2004; Scherer, Müller, Neuper, Graimann, \& Pfurtscheller, 2004; Ramsey, van De Heuvel, Kho, \& Leijten, 2006; Mellinger et al., 2007). Though these 'classic' BCls (mostly based on electroencephalography [EEG]) have already been applied successfully in affected patients (Birbaumer et al., 1999; Kübler et al., 1999; Hochberg et al., 2006, 2012; Nijboer et al., 2008), not all individuals achieve proficiency in EEG-based $\mathrm{BCl}$ control (a phenomenon coined ' $\mathrm{BCl}$ illiteracy'). Therefore, exploiting hemodynamic brain signals as measured with $\mathrm{fMRI}$ or functional near-infrared spectroscopy (fNIRS) has been suggested as an alternative approach (Weiskopf et al., 2003). One important aspect when developing communication and control $\mathrm{BCls}$ is to try to increase the degrees of freedom in encoding different intentions, i.e., to allow the $\mathrm{BCl}$ user to choose from as many as possible options. One necessity in this context is to enable the $\mathrm{BCl}$ user to voluntarily evoke just as many differentiable brain states (e.g., distinct fMRI brain-activation patterns). But how can this be achieved?

Several approaches have been explored in the context of fMRI-based brain-computer interfacing: a first approach employed the modulation of spatial BOLD-signal features for encoding separate intentions by implementing different mental tasks (and thereby evoking spatially different brain-activation patterns). This possibility was tested in several fMRI experiments including proof-of-principle 
studies with healthy participants (Lee, Ryu, Jolesz, Cho, \& Yoo, 2009; Yoo et al., 2012) and clinical studies involving patients suffering from a disorder of consciousness in order to detect residual consciousness awareness (Owen et al., 2006; Monti et al., 2010). In one study, healthy participants navigated through a two-dimensional (2D) virtual maze by performing a specific mental task (eliciting a unique brain activation pattern) for each of the four movement directions ("right", "left", "up", and "down") (Yoo et al., 2004). In a later follow-up study, it was shown that this procedure also enables adequate control over 2D movements of a robotic arm (Lee et al., 2009). Note however, that the amount of mental tasks suited for encoding different intentions seems to be rather limited when using MRI scanners with conventional field strengths (1.5T or $3 \mathrm{~T}$ ). So far, the most successfully implemented mental tasks in this context are motor imagery, spatial navigation, mental calculation, and inner speech (Yoo et al., 2004; Owen et al., 2006; Boly et al., 2007; Lee et al., 2009). As a second approach to increase the degrees of freedom in encoding separate intentions, researchers have explored the possibility to systematically vary temporal BOLD-signal features (i.e., using different encoding time intervals) (Sorger et al., 2009; Bardin et al., 2011). Finally, a combinatory use of both spatial and temporal BOLD-signal features was successfully tested and further developed to allow for encoding all letters of the English alphabet and the blank space enabling fMRI-based free letter spelling (Sorger, Reithler, Dahmen, \& Goebel, 2012). Theoretically (and as a third option), it might be feasible to hemodynamically encode separate intentions by systematically varying the BOLD-signal level (i.e., exploiting magnitudinal BOLD-signal features) within the same region of interest (ROI). The ability to differentially modulate the BOLD-signal level might be given a priori when instructing participants appropriately. However, providing neurofeedback on the current brain activation level might further enhance the gradual self-regulation performance. Magnitudinal BOLD-signal features have been employed previously in a real-time 'brain pong' hyperscanning study (Goebel, Sorger, Kaiser, Birbaumer, \& Weiskopf, 2004) where two interacting participants played pong by controlling the vertical position of their rackets by modulating the level of regional brain activation. In this game-like situation, gradual self-regulation of the BOLD signal was, however, not systematically investigated. Based on the presented background, the current feasibility study investigated systematically whether healthy participants are able to gradually modulate the 
BOLD-signal level by employing different mental strategies and whether fMRI-based neurofeedback can facilitate the presumed gradual self-regulation ability (in the following coined instantaneous feedback effect to differentiate it from a, e.g., feedback transfer effect).

In order to answer the abovementioned questions, participants were trained to modulate their BOLD-signal magnitude to different target levels without and with the support of rt-fMRI neurofeedback about the BOLD-signal level in a predefined mental task related brain region.

The main hypotheses of the current study were:

1. The BOLD-signal level can be self-regulated gradually (gradual selfregulation effect).

2. The availability of neurofeedback about the current BOLD-signal level further improves the gradual self-regulation performance (instantaneous feedback effect).

\section{EXPERIMENTAL PROCEDURES}

\section{Participants}

Ten healthy participants (age: $27 \pm 3.8$ years, five female, one left-handed), all students or staff members of the Faculty of Psychology and Neuroscience at Maastricht University with normal or corrected-to-normal vision participated in the study (see Table 1 for participants' characteristics). None of the participants had participated in a neurofeedback experiment before. Before each MRI scanning session, participants gave written informed consent. The experimental procedure was approved by the local Ethics Committee of the Faculty of Psychology and Neuroscience at Maastricht University. 
Table 1. Participants' characteristics and methodological details.

\begin{tabular}{c||c|c|c|c|c}
\hline Participant & Sex & Age & $\begin{array}{c}\text { Condition of } \mathbf{1}^{\text {st }} \\
\text { MRI session }\end{array}$ & $\begin{array}{c}\text { MRI } \\
\text { scanner } \\
\text { (field } \\
\text { strength) }\end{array}$ & $\begin{array}{c}\text { Activation } \\
\text { strategy } \\
\text { (mental task) }\end{array}$ \\
\hline \hline P01* & male & 24 & feedback & $3 T$ & inner speech \\
\hline P02 & male & 27 & feedback & $1.5 T$ & $\begin{array}{c}\text { mental } \\
\text { orchestra }\end{array}$ \\
\hline P03 & female & 32 & no feedback & $1.5 T$ & inner speech \\
\hline P04 & female & 35 & no feedback & $1.5 T$ & $\begin{array}{c}\text { visual motion } \\
\text { imagery }\end{array}$ \\
\hline P05 & female & 25 & no feedback & $1.5 T$ & inner speech \\
\hline P07 & male & 25 & feedback & $3 T$ & mental drawing \\
\hline P08 & male & 23 & feedback & $3 T$ & inner speech \\
\hline P09 & female & 25 & no feedback & $1.5 T$ & mental running \\
\hline female & 26 & no feedback & $3 T$ & inner speech \\
\hline
\end{tabular}

Remark: * left-handed.

\section{Experimental design}

Participants were asked to modulate their BOLD signal to three different target levels. Importantly, participants received no feedback in one $\mathrm{fMRI}$ session, whereas in the other session they were provided with neurofeedback information on the current BOLD-signal level in a pre-defined mental task-related brain region. Thus, we employed a two-way within-subject design with target level (low, medium and high) and type of training (no feedback and feedback) as factors. For each participant, the no-feedback and feedback fMRI sessions were on separate days. Note that the order of the 'type of training' conditions ('no feedback-feedback' or 
'feedback-no feedback') was balanced across participants (see Table 1) in order to exclude potential confounds. Both scanning sessions consisted of four training (modulation) runs (see Fig. 1) in which participants were visually instructed to modulate their BOLD-signal magnitude to the three different target levels. Each 'target level' condition appeared three times per run in randomised order resulting in a total of twelve trials per 'target level' and 'type of training' condition. The duration of the nine modulation trials per run as well as of the intermingled ten resting periods was $26 \mathrm{~s}$ resulting in a modulation run length of $8 \mathrm{~min}$ and $14 \mathrm{~s}$. A feedback scanning session started with a functional localiser run in order to select a mental task-specific neurofeedback target region. In the functional localiser, two target levels (50\% and 100\%) were implemented (five trials per 'target level' condition). The two 'target level' conditions appeared in alternating order. Again, the duration of the (ten) modulation trials and the (11) resting periods were $26 \mathrm{~s}$ adding up to a total run duration of $9 \mathrm{~min}$ and $6 \mathrm{~s}$.

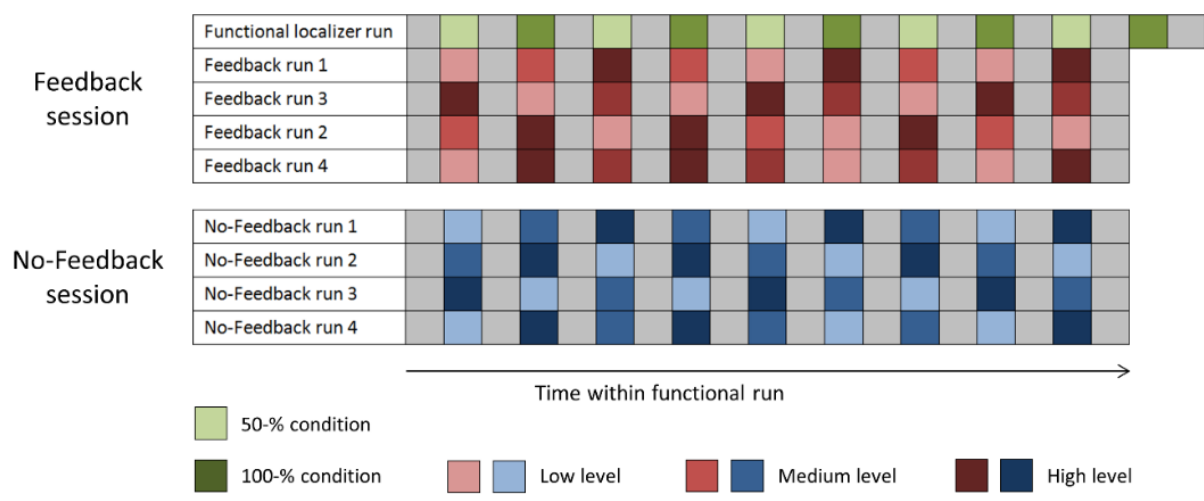

Figure 1: Overview of experimental design. The figure depicts the experimental design for one participant. Bluish and reddish colours indicate 'no feedback' and 'feedback' conditions, respectively. Greenish colours refer to the two conditions implemented in the functional localiser run. Resting blocks are indicated by grey cells. Resting and modulation blocks took $26 \mathrm{~s}$ each.

\section{Visual instruction and neurofeedback presentation}

In order to instruct participants, a thermometer-like display on black background was used consisting of ten white rectangles stacked on top of each other (see Fig. 2). To instruct participants to adjust their BOLD signal to a particular target 
level, the outline of a certain rectangle turned red for the duration of the modulation trial. Thus, the vertical position of the coloured rectangle represented the desired target level.

In the functional localiser run, rectangle 5 (counted from bottom) corresponded to the $50 \%$ condition and rectangle 10 represented the $100 \%$ condition. In the modulation runs, rectangles 3,6 , and 9 , corresponded to the low, medium and high 'target level' conditions, respectively. During resting periods, no rectangle was coloured red.

In the modulation runs of the feedback session, participants were additionally provided with continuously updated gradual information about their current BOLD-signal level within the neurofeedback target region. This was realised by filling in (with grey colour) the thermometer's rectangles in such a way that the vertical position in the display corresponded to the actual BOLD-signal level within the neurofeedback target region. Note that the neurofeedback display was kept as intuitive as possible assuming that a straightforward interpretation of the neurofeedback information facilitates learning (Weiskopf, Scharnowski, et al., 2004).

Visual stimulation was generated by a personal computer (PC) using custom-made software and projected onto a frosted screen located at the end of the scanner bore (at the side of the participant's head) with a liquid crystal display (LCD) projector. Participants viewed the screen via a mirror mounted to the head coil at an angle of $\sim 45^{\circ}$. 


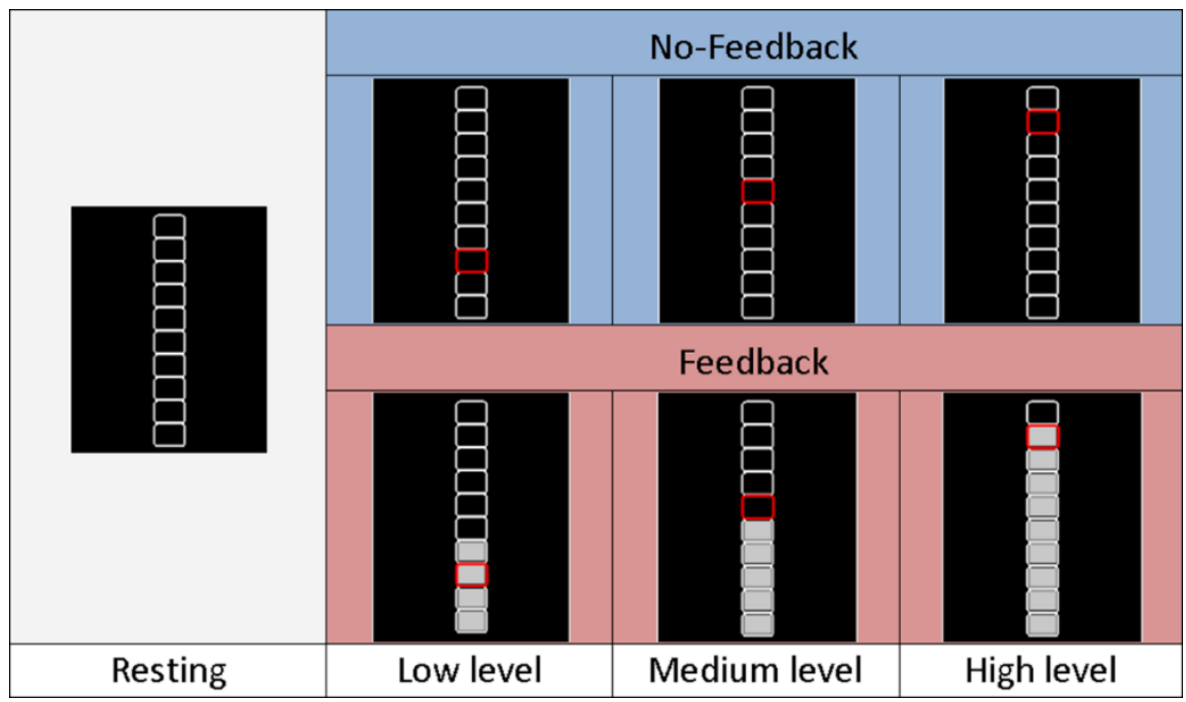

Figure 2: Visual instruction and neurofeedback display. A thermometer-like display on black background was used consisting of ten white rectangles stacked on top of each other. To instruct participants to adjust their BOLD-signal to a particular target level, the outline of a certain rectangle turned red for the duration of the modulation trial. During resting blocks no rectangle was coloured red. During feedback runs, continuously updated gradual feedback information was additionally provided by filling the rectangles with grey colour according to the current BOLD signal intensity reached by the participant in the neurofeedback target region.

\section{General procedure}

\section{Preparation}

At the beginning of the first session, participants were familiarised with the general idea of the study (investigating the ability to reach different brain activation levels without and with neurofeedback). They were introduced to the fMRI-neurofeedback concept and methodology and the general procedure of the current study. Furthermore, participants were familiarised with the neurofeedback display, hemodynamic delay and noise level of fMRI signals. Finally, they were instructed to avoid body movements while lying in the MRI scanner.

\section{Suggestion and selection of activation and modulation strategies}

Experimenters suggested various mental tasks (inner speech, motor imagery, mental calculation, visual imagery and auditory imagery) that had been proven to 
evoke robust brain activation in circumscribed brain regions in previous $\mathrm{fMRI}$ studies (e.g., Yoo et al., 2004; Owen et al., 2006; Boly et al., 2007; Lee et al., 2009; Sorger et al., 2009, 2012; Monti et al., 2010; Bardin et al., 2011) as possible activation strategies. Additionally, the experimenters recommended several modulation strategies that could be applied by participants to alter the brain activation level. Basically, these strategies allowed for changing certain aspects of mental task performance parametrically (e.g., the speed, intensity or complexity). The modulation strategies were either based on neuroscientific pre-knowledge, i.e., studies showing parametric effects on brain activation by systematically changing aspects of mental task performance (e.g., Culham \& Kanwisher, 2001; Shergill et al., 2002; Berman et al., 2012; Lipp et al., 2012) or on naïve hypotheses of the experimenters on how the BOLD signal might be altered.

Participants were asked to choose an individual activation strategy which they could execute continuously and manipulate by applying the modulation strategies suggested by the experimenter (see above). Participants selected their activation strategies and initial modulation strategies based on personal preference or feeling of best mastery.

\section{Task instruction}

Participants were instructed to keep their selected activation strategy constant across all functional runs (functional localiser, 'no feedback' and 'feedback' runs). Thus, they should not change their general activation strategy across time (and sessions). In order to modulate their BOLD signal to the different target levels, participants were asked to apply the modulation strategies. Importantly, in the feedback condition, participants were instructed to consider the provided neurofeedback information and to explore which of the modulation strategies were most effective. Moreover, participants were explicitly allowed to adapt the suggested modulation strategies or even generate and test novel ('own') modulation strategies. During functional localiser and 'no feedback' runs, participants were asked to try to evoke different brain activation levels based on their current hypothesis on how the BOLD-signal magnitude can be altered systematically. 


\section{Data acquisition}

\section{MRI data acquisition}

MRI data were obtained using a 1.5T whole-body (Magnetom Sonata; Siemens AG, Erlangen, Germany) and a 3T head scanner (Siemens Allegra, Siemens AG) (see Table 1). Participants were placed comfortably in the MRI scanner; their heads were fixated with foam padding to minimise spontaneous or task-related motion.

\section{Functional measurements}

Repeated single-shot echo-planar imaging (EPI) was performed using the BOLD effect as an indirect marker of local neuronal activity (Ogawa, Lee, Kay, \& Tank, 1990). Except for the number of acquisitions (functional localiser run: 273 volumes; modulation runs: 247 volumes), identical scanning parameters were used for all functional measurements (repetition time $[T R]=2000 \mathrm{~s}$, echo time $[T E]=40 \mathrm{~ms}$, flip angle $[\mathrm{FA}]=90^{\circ}$, field of view $[\mathrm{FOV}]=224 \times 224 \mathrm{~mm}^{2}$, matrix size $=64 \times 64$, number of slices $=25$, slice thickness $=3 \mathrm{~mm}, 1 \mathrm{~mm}$ gap, slice order $=$ ascending $/$ interleaved) . In the feedback sessions, functional images were reconstructed and written to the scanner console's hard disk in real time using a custom-made image export running on the image reconstruction computer (implemented in Siemens ICE VA30) (Weiskopf, Mathiak, et al., 2004; Weiskopf, Klose, Birbaumer, \& Mathiak, 2005). The real-time data analysis software (see below) running on a separate PC retrieved the image files via local area network (LAN) and a Windows drive map as soon as they were created by the image reconstruction system.

\section{Anatomical measurements}

Each participant underwent a high-resolution T1-weighted anatomical scan using a three-dimensional (3D) magnetization-prepared rapid-acquisition-gradient-echo (MP-RAGE) sequence (1.5-T scanning: 192 slices, slice thickness $=1 \mathrm{~mm}$, no gap, $\mathrm{TR}=2000 \mathrm{~ms}, \mathrm{TE}=3.93 \mathrm{~ms}, \mathrm{FA}=15^{\circ}, \mathrm{FOV}=250 \times 250 \mathrm{~mm}^{2}$, matrix size $=256 \times 256$, total scan time $=8 \mathrm{~min}$ and 34s; 3-T scanning: 192 slices, slice thickness $=1 \mathrm{~mm}$, no gap, $\mathrm{TR}=2250 \mathrm{~ms}, \mathrm{TE}=2.6 \mathrm{~ms}, \mathrm{FA}=9^{\circ}, \mathrm{FOV}=256 \times 256 \mathrm{~mm} 2$, matrix size $=256 \times 256$, total scan time $=8 \mathrm{~min}$ and $26 \mathrm{~s}$ ). 


\section{Acquisition of physiological data}

In order to assess potential cardiorespiratory effects on the fMRI signal level, heart and breathing rates of the participants were recorded during all feedback runs using the scanner's standard MRI-compatible pulse oximeter and chest band. Due to technical limitations, acquisition of physiological data was only feasible for the feedback runs in the 1.5T measurements (five participants).

\section{Acquisition of introspective data}

After scanning, participants filled in a post-hoc questionnaire obtaining precise descriptions of the applied activation and modulation strategies as well as other relevant information (e.g., subjective experience with neurofeedback).

\section{Data Analysis}

\section{(f)MRI data analysis}

Online/real-time analysis of fMRI data

Functional data of the feedback session were analysed using real-time data analysis software (Turbo-BrainVoyager, Brain Innovation B.V., Maastricht, the Netherlands) in order to a) select and define the neurofeedback target region and b) generate the neurofeedback information.

Selection and definition of neurofeedback target regions: After completion of the functional localiser run, the first two volumes were discarded from further analysis to account for T1-saturation effects. Functional data were then pre-processed (motion correction, linear trend removal, temporal high-pass filtering [three cycles/time course]). Eventually, a multiple regression general linear model (GLM) was calculated voxel-wise applying predictors corresponding to the two 'target level' conditions (predictor time courses being derived from a boxcar function convolved with a standard hemodynamic response function [single-gamma function (Boynton, Engel, Glover, \& Heeger, 1996)].

Candidate neurofeedback target regions were identified by contrasting the mean brain activation during both 'target level' conditions to the mean activation during the interleaved resting periods. From the obtained F-maps $(p<0.05$, Bonferroni-corrected), a region of interest (ROI) was defined for each participant individually as neurofeedback target region based on the following criteria: 
1. The region's signal time course should be reliable and robust, demonstrating a typical hemodynamic response shape across the entire functional run and small standard errors when averaging across repetitions.

2. The region should present a strong $\mathrm{fMRI}$ response (high maximal \% BOLDsignal change relative to baseline and high signal-to-noise ratio).

3. Brain regions should be known to be involved in the performance of the selected activation strategy, e.g., Broca's area during inner speech (Shergill et al., 2002) or premotor areas during motor imagery (Guillot et al., 2008) should be preferred (implementation of a priori knowledge).

4. The region should be relatively insensitive to susceptibility artefacts.

5. The region should comprise about 10-15 neighbouring voxels across up to three separate $\mathrm{fMRI}$ slices.

Maximal \% BOLD-signal values of the selected neurofeedback target regions were calculated and noted down as they were needed for calculating the neurofeedback information.

Generation of the neurofeedback information: After the first two volumes were discarded from further analysis, the data of the feedback runs were analysed in real time. The computational steps described in the following were performed as soon as the necessary data were available and had been spatially aligned to the first volume of the functional localiser run in order to correct for potential head movement. In order to generate the neurofeedback information, a baseline was determined as the mean of the five data points prior to the onset of the first modulation trial. The baseline was continuously updated before each new modulation trial (sliding baseline). Eventually, the neurofeedback information was calculated separately for each functional volume by:

1. Extracting and averaging the BOLD-signal values of all voxels composing the neurofeedback target region.

2. Normalizing the resulting mean value to $\%$ BOLD-signal change with respect to the corresponding baseline level.

3. Calculating the level ratio (LR) by relating the $\%$ BOLD-signal change value of the current time point $i\left(\% B O L D_{i}\right)$ to the maximal \% BOLD-signal value 
(\% BOLD ${ }_{\max }$ ) obtained from the functional localiser run $\left(L R=\% B O L D_{i} / \% B O L D_{\text {max }}\right)$. The resulting value was clipped to the range [0.0-1.0] corresponding to the baseline level and the maximum level achieved in the functional localiser run, respectively. Values below 0.0 and above 1.0 were displayed as 0.0 and 1.0 , respectively.

4. Relating the level ratio to the number of rectangles to be coloured grey $\left(\mathrm{N}_{\text {filled }}\right)$ by linear transformation $\left(\mathrm{N}_{\text {filled }}=\right.$ round $(10 \times \mathrm{LR})$ ).

Thus, an activation level of half the maximum activation ( $L R=0.5)$, for example, was represented by five grey rectangles (filled from bottom) within the thermometer-like neurofeedback display. Neurofeedback information was immediately presented to the participant and was continuously updated every $2000 \mathrm{~ms}$ (i.e., each functional volume).

Offline analysis of (f)MRI data

Post-hoc analysis of the (f)MRI data was done using BrainVoyager QX (v2.8, Brain Innovation, Maastricht, the Netherlands) and SPSS 21 (SPSS Inc., Chicago, IL, USA).

Analysis of anatomical data

Obtained anatomical data sets were first corrected for spatial intensity inhomogeneity. For each participant, the data set from the first session was transferred into ACPC space. Subsequently, the data set from the second session was automatically aligned to the ACPC version of the first data set. Finally, both data sets were spatially normalised by Talairach transformation.

Analysis of functional data

Pre-processing: All functional data sets underwent standard pre-processing optimised for the current experiment. Slice scan time correction and temporal high-pass filtering (three cycles per time course) was performed to account for temporal differences in slice acquisition and to remove low-frequency drifts, respectively. Furthermore, 3D head-motion detection and correction was applied by spatially aligning all functional volumes of a session to the first functional volume of the first run within that session. Finally, all functional runs were spatially 
normalised to Talairach space and interpolated to a $3 \mathrm{~mm}^{3}$ voxel resolution. The individual neurofeedback target regions were transformed into 3D volumes of interest (VOIs) in Talairach space.

Group analysis of mean betas: A VOI-based random-effects group GLM analysis (standard feature implemented in BrainVoyager QX) was carried out. The GLM included predictors for each 'target level' condition (low, medium, and high), 'type of training' condition ('no feedback' and 'feedback'), and six motion parameters (three rotations and three translations) as confounding predictors. Condition effects were modelled using a boxcar function, which was convolved with the two-Gamma hemodynamic impulse function (Friston et al., 1998) to take into account the hemodynamic response delay. Beta values for each 'target level' condition were calculated separately for each 'type of training' condition by fitting the GLM to the average BOLD-signal time course within the individual VOIs. Based on the resulting betas, a two-way repeated measures analysis of variance (ANOVA, F-Test) with factors for 'target level' and 'type of training' was performed to test the interaction hypothesis. Furthermore, a contrast analysis was carried out, specifically testing the differences between 'target level' conditions within a 'type of training' condition. Obtained $\mathrm{p}$-values were evaluated against a one-sided threshold of $\alpha=0.05$, as a directed hypothesis of the gradual self-regulation and instantaneous feedback effect was posed a priori.

Single-trial analysis: In order to extract single-trial beta values for all modulation ('no feedback' and 'feedback') trials, a VOI-based GLM (including the six motion predictors as confounding predictors next to the single-trial predictors) was carried out separately for each functional run. Single-trial beta values were calculated by fitting the GLM to the average BOLD-signal time course within the individual VOIs. The resulting single-trial beta values were correlated (Pearson's correlation coefficient) with the particular target level for both 'type of training' conditions separately and a Fisher z-transformation was applied to each correlation coefficient. This resulted in two Fisher z-transformed correlation coefficients for each participant, one for the 'no feedback' and one for the 'feedback' condition. Subsequently, a one-sided paired t-test was carried out, comparing the correlation 
coefficients between the two sessions against a threshold of $\alpha=0.05$ (reasoning see above).

Single-subject analysis: In order to test the ability of each individual participant to gradually modulate regional brain activation to the target levels in the 'no feedback' and 'feedback' condition, the individual VOI-specific beta values for each combination of 'target level' and 'type of training' conditions were entered in a contrast analysis, testing whether the brain activation levels can be significantly differentiated in both the 'type of training' conditions. Obtained p-values were evaluated against a one-sided threshold of $\alpha=0.05$.

\section{Physiological data}

Acquired heart and breathing rates were analysed using in-house software written in MATLAB (v6.5 R13; The MathWorks, Natick, USA). Mean values and standard errors were calculated separately for the 'target level' conditions and the resting condition.

\section{Introspective data}

The post-hoc questionnaires that participants filled in after each scanning session were qualitatively analysed to gain insights in the participant's phenomenological experience of the selected activation and modulation strategies.

\section{RESULTS}

\section{Introspective results}

The individually-chosen activation strategies (mental tasks; see Table 1) considerably varied across participants and can be classified into four categories: inner speech, motor imagery, auditory imagery, and visual imagery.

Inner speech: Five participants (P01, P03, P05, P07, P10) chose inner speech as their activation strategy. They either recited a given text (e.g., a poem, a prayer) or spontaneously generated speech silently. Strategies to modulate the BOLD-signal level included: a) making the content of the inner speech more complex (naming single words to generating whole sentences), b) speaking at a different pace (very 
slow to extremely fast), or c) varying sound intensity (almost silent to extremely loud).

Motor imagery: Two participants performed a motor imagery task (mental drawing [P06] and mental running [P09]). Modulation strategies involved systematically varying the rhythm of the movement, the environment in which the movement was embedded (e.g., from running in a calm environment to running together with several people, culminating in running in a competition), and the pace of movement.

Auditory imagery. Two participants performed an auditory imagery task. One participant (P02) mentally conducted an orchestra (mental orchestra) and changed the pace, rhythm and sound level of the music as well as number of orchestra instruments to vary the BOLD-signal level. The second participant (P08) imagined simple sounds (mental sounds) varying the rhythmicity of the tones (no rhythm to high rhythmical variations) in order to adapt the BOLD-signal level.

Visual imagery. One participant (P04) performed visual motion imagery. The participant imagined a vertically jumping object and changed frequency and rhythm of the object's motion as modulation strategy.

In general, participants reported to have been able to apply their selected activation strategy easily and that it was possible to additionally apply modulation strategies (i.e., vary the content of the imagination). They also indicated to have been able to attend and react to the neurofeedback display changes in the 'feedback' condition and that modifying the modulation strategy to some extent was also represented in a change in the neurofeedback signal. Generally, the 'feedback' condition was perceived enjoyable though being more demanding and requiring more attentional resources. Especially the lowest modulation level seemed to be difficult to obtain for some participants. Most importantly, participants reported that some of the initial modulation strategies were quite effective but that the provision of neurofeedback helped them to further optimise (fine-tune) the modulation strategies or to even elaborate new strategies. For example, P03 (using inner speech as activation strategy) employed a systematic variation of the speech rate as modulation strategy. In the 'feedback' condition, she realised that using an unnaturally low speech rate did not result in a low BOLD-signal level. Accordingly, she adapted her initial modulation strategy - using 
finally a normal, fast and very fast speech rate to achieve a low, medium and high BOLD-signal level, respectively.

\section{fMRI results}

\section{Neurofeedback target regions}

For each participant, a neurofeedback target region fulfilling the abovementioned criteria could be determined based on the functional localiser data obtained during the feedback session (see Fig. 3). Characteristics of the selected ROIs (anatomical labelling, size, Talairach coordinates etc.) can be derived from Table 2.

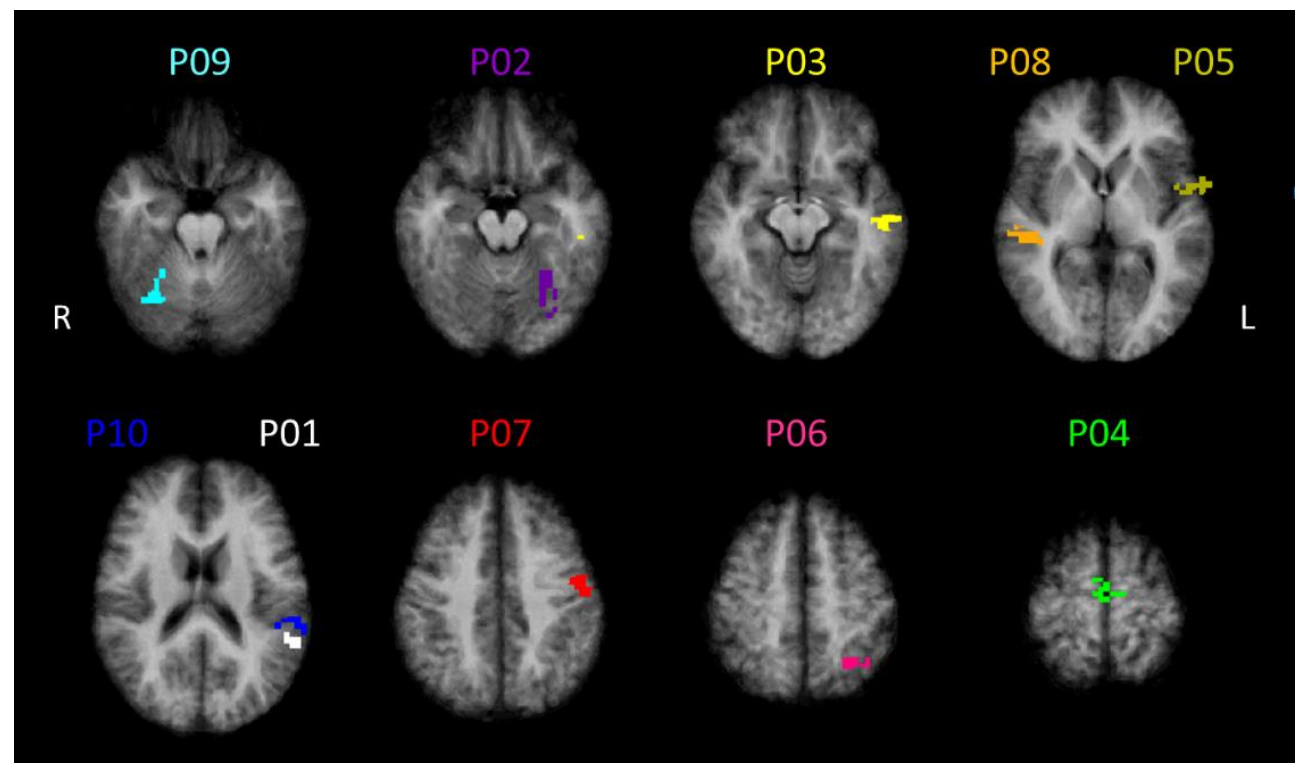

Figure 3: Individual neurofeedback target regions. The figure shows the individually defined neurofeedback target regions overlaid on transversal slices of the participants' mean anatomy in Talairach space. Note that the selected regions are the widely distributed across the whole cortex. Characteristics of the selected brain regions (anatomical labelling, size, Talairach coordinates etc.) can be derived from Table 2.

Abbreviations: $L=$ left hemisphere; $R=$ right hemisphere. 
Table 2. Characteristics of neurofeedback target regions.

\begin{tabular}{|c|c|c|c|c|c|c|}
\hline \multirow{2}{*}{$\begin{array}{c}\text { Participant } \\
\text { P01* }\end{array}$} & \multirow{2}{*}{$\begin{array}{c}\begin{array}{c}\text { Anatomical } \\
\text { label }\end{array} \\
\text { STG }\end{array}$} & \multirow{2}{*}{$\begin{array}{c}\begin{array}{c}\text { Size } \\
\left(\mathrm{mm}^{3}\right)\end{array} \\
511\end{array}$} & \multicolumn{3}{|c|}{$\begin{array}{l}\text { Talairach } \\
\text { coordinates } \\
(x, y, z)\end{array}$} & \multirow{2}{*}{$\begin{array}{c}\begin{array}{c}\text { Maximal \% BOLD- } \\
\text { signal value }\end{array} \\
5.0\end{array}$} \\
\hline & & & -57 & -41 & 16 & \\
\hline P02 & FG & 673 & -27 & -59 & -15 & 2.0 \\
\hline P03 & MTG & 654 & -53 & -18 & -8 & 2.0 \\
\hline P04 & SFG & 471 & 0 & -11 & -59 & 2.0 \\
\hline P05 & IFG & 481 & -56 & 3 & 4 & 3.0 \\
\hline P06 & SPL & 614 & -31 & -55 & 50 & 4.0 \\
\hline P07 & preCG & 447 & -49 & -8 & 41 & 3.0 \\
\hline P08 & STG & 774 & 48 & -26 & 4 & 2.0 \\
\hline P09 & FG & 526 & 28 & -57 & -19 & 5.0 \\
\hline P10 & SMG & 882 & -51 & -33 & 17 & 2.0 \\
\hline
\end{tabular}

Remark: * left-handed.

Abbreviations: $F G=$ fusiform gyrus, IFG = inferior frontal gyrus, STG = superior temporal gyrus, MTG = middle temporal gyrus, preCS = precentral Gyrus, SFG = superior frontal gyrus, SMG = supramarginal gyrus, $S P L=$ superior parietal lobule.

\section{Gradual self-regulation effect ('no feedback' data)}

Group results

Across participants, mean beta values significantly increased with each target level step in a linear way ( $p<0.001$; see Fig. 4 A and Fig. 4B). Fitting a linear trend line to the obtained mean target level beta values showed a clear linear modulation of the brain activation level $\left(R^{2}=0.888\right.$, see Fig. $\left.4 A\right)$. Moreover, contrast analyses showed that two hypothesised 'between target level contrasts' were significant (high vs. 
medium level and low level vs. resting; $\mathrm{p}<0.01)$. No difference could be obtained for contrasting the medium vs. low 'target level' condition ( $p=0.22$, Fig. 4B).

\section{Single subject's results}

When looking at the number of significant 'between target level contrasts' (in the desired direction), $80 \%$ of participants (P01, P03, P04, P06-10) were able to gradually modulate local brain activation to at least two target levels in the 'no feedback' condition (see second column of Table 3). Using a stricter criterion, namely a significant correlation of the single-trial beta values and the activation target levels, actually $60 \%$ of participants (P03, P06-P10) were able to gradually modulate the brain activation level in the absence of neurofeedback (see third column in Table 3). In Fig. 4C, mean beta values of the 'no feedback' conditions are plotted separately for each participant.

$\rightarrow$

Figure 4: Gradual self-regulation ability across both 'type of training' conditions (group and single-subject results). A. Mean beta values for each 'target level' condition across all participants separately for the 'no feedback' (blue) and 'feedback' (red) condition. Error bars represent standard errors of the means from within-subjects analysis. B. Contrast analysis between target level-specific beta values separately for the 'no feedback' and 'feedback' condition across all participants. All comparisons reach statistical significance $(p<0.01$, see asterisks) except for one contrast (contrasting the medium vs. the low target level in the 'no feedback' condition). C. Single-subject mean beta values separately for each 'target level' and 'type of training' condition. Participants with a black underline underwent 'feedback' condition first and 'no feedback' condition second) 


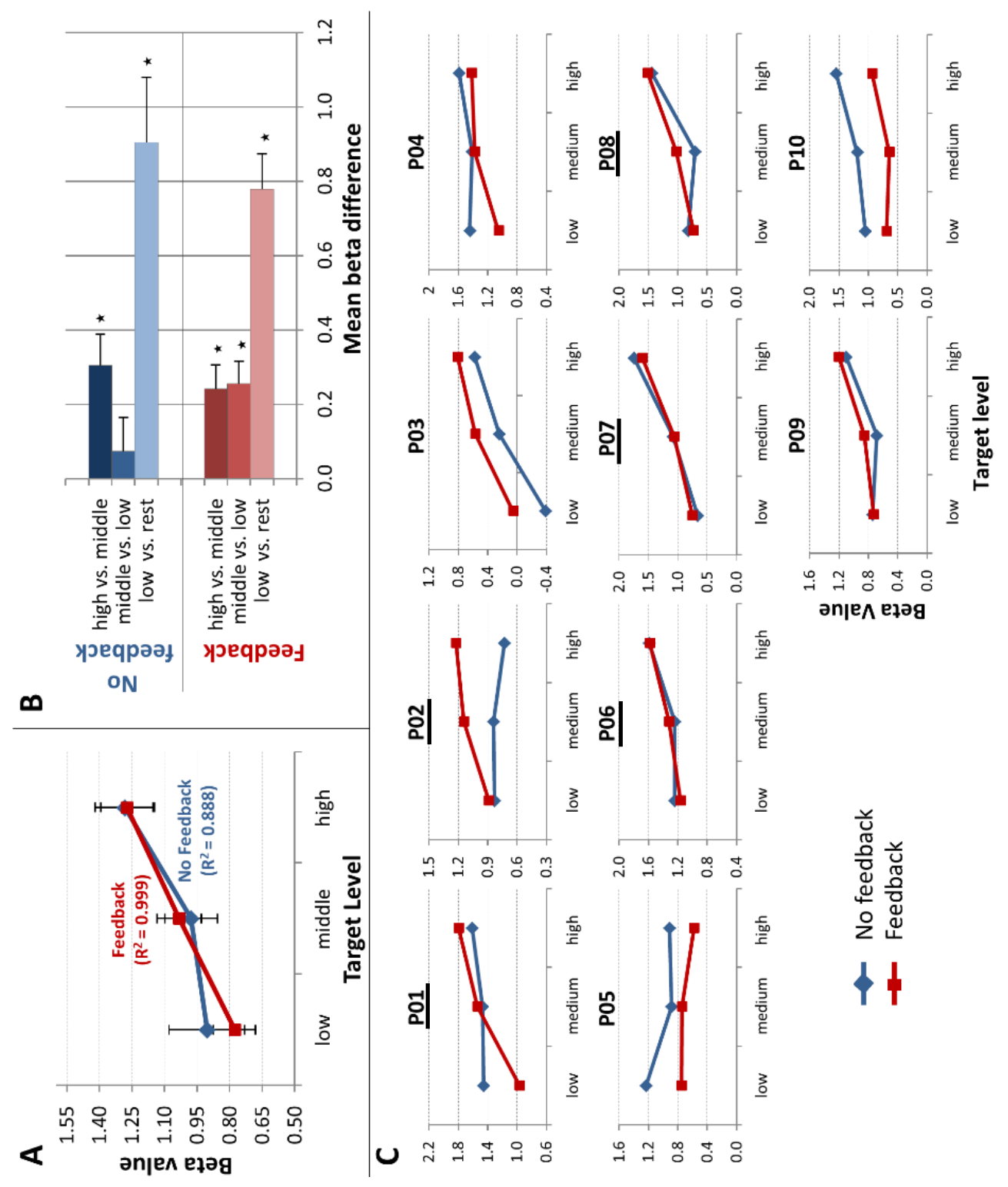


Table 3. Number of significant between-target level contrasts and correlations of single-trial beta values and target levels separately for the two 'type of training' conditions per participant.

\begin{tabular}{|c|c|c|c|c|}
\hline \multirow[t]{2}{*}{ Participant } & \multicolumn{2}{|c|}{ No-Feedback condition } & \multicolumn{2}{|c|}{ Feedback condition } \\
\hline & $\begin{array}{c}\text { Number of } \\
\text { significant } \\
\text { contrasts }\end{array}$ & $\begin{array}{l}\text { Correlation of } \\
\text { single-trial beta } \\
\text { values and } \\
\text { target levels }\end{array}$ & $\begin{array}{l}\text { Number of } \\
\text { significant } \\
\text { contrasts }\end{array}$ & $\begin{array}{c}\text { Correlation of } \\
\text { single-trial beta } \\
\text { values and } \\
\text { target levels }\end{array}$ \\
\hline$\underline{\mathrm{P} 01}$ & 2 & 0.188 & $3(+)$ & $0.745 * *(\uparrow)$ \\
\hline$\underline{\mathrm{P} 02}$ & 1 & -0.083 & $2(+)$ & $0.355 *(\uparrow)$ \\
\hline P03 & 2 & $0.415 * *$ & $2(=)$ & $0.677^{* *}(\uparrow)$ \\
\hline P04 & 2 & 0.094 & $2(=)$ & $0.402 * *(\uparrow)$ \\
\hline P05 & 1 & $-0.326^{(* *)}$ & $1(=)$ & $-0.152(\uparrow)$ \\
\hline$\underline{\mathrm{P} 06}$ & 2 & $0.287 *$ & $3(+)$ & $0.411 * *(\uparrow)$ \\
\hline$\underline{\mathrm{P} 07}$ & 3 & $0.778 * *$ & $3(=)$ & $0.824 * *(\uparrow)$ \\
\hline$\underline{\mathrm{P} 08}$ & 2 & $0.455 * *$ & $3(+)$ & $0.495 * *(\uparrow)$ \\
\hline P09 & 2 & $0.531 * *$ & $2(=)$ & $0.41 * *(\downarrow)$ \\
\hline P10 & 2 & $0.501 * *$ & $2(=)$ & $0.266(\downarrow)$ \\
\hline Mean & 1.9 & 0.284 & 2.3 & 0.443 \\
\hline
\end{tabular}

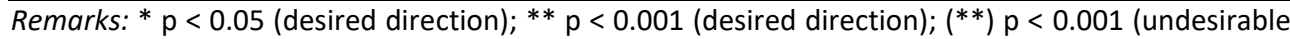
direction); participants with a black underline underwent the 'feedback condition' first and the 'no feedback' condition second; $(\uparrow)$ or $(\downarrow)$ indicates a higher or a lower correlation coefficient in the 'feedback' condition ( $v s$. in the 'no feedback' condition); (+) or (=) indicates more or the same number of significant 'between target level contrasts'. 


\section{Instantaneous feedback effect}

Single-subjects results

Four participants showed a higher number of significant 'between target level contrasts' in the 'feedback' compared to the 'no feedback' condition (P01, P02, P06, P08). The remaining six participants (P03-P07, P09, P10) showed the same amount of significant 'between target level contrasts' for both 'type of training' conditions (see fourth column in Table 3). Calculating the differences between the Fisher z-transformed correlations (of the single-trial beta values and the activation target levels) for the 'feedback' and 'no feedback' data show that in $80 \%$ of the participants, receiving neurofeedback (vs. not receiving neurofeedback) led to an increased association between the single-trial modulation of regional brain activation and the desired target level (see fifth column of Table 3).

In Fig. 4C, mean beta values for both the 'no feedback' and the 'feedback' conditions are plotted separately for each participant. One participant (P05) did not seem capable of performing target level-specific adjustments of the BOLD-signal level independent of 'type of training' condition. However, neurofeedback seemed to weaken the negative association observed in the 'no feedback' condition (see Fig. 4C, P05). In two participants (P09 and P10), providing neurofeedback seemed to rather impede the ability to gradually modulate the brain activation level which these participants were presenting in the (preceding) 'no feedback' condition (see Fig. 4C, P10).

\section{Group results}

In the ROI-based random-effects group analysis, the interaction effect between the factors 'target level' and 'type of training' just missed significance $(p=0.083)$. However, the correlation of the single-trial beta values and the activation target levels was significantly higher in the 'feedback' than in the 'no feedback' condition $(p<0.05)$. Fitting a linear trend line to the target level beta values across participants showed that providing neurofeedback led to an almost linear modulation of brain activation within the neurofeedback target regions $\left(R^{2}=0.999\right)$, while in the 'no feedback' condition, the linear modulation was lower to some degree $\left(R^{2}=0.888\right.$ ) (see Fig. $\left.4 A\right)$. This becomes also obvious by inspecting Fig. $4 \mathrm{~B}$ showing increased equidistance of target level - beta value differences in the 'feedback' ( $v s$. 'no feedback') condition. Finally, contrast analyses showed that 
in the 'feedback' condition, each target level beta value was significantly different from the other target level beta values and from rest ( $p<0.002$, Fig. 4B) while in the 'no feedback' condition, only two of these contrasts were significant (see above).

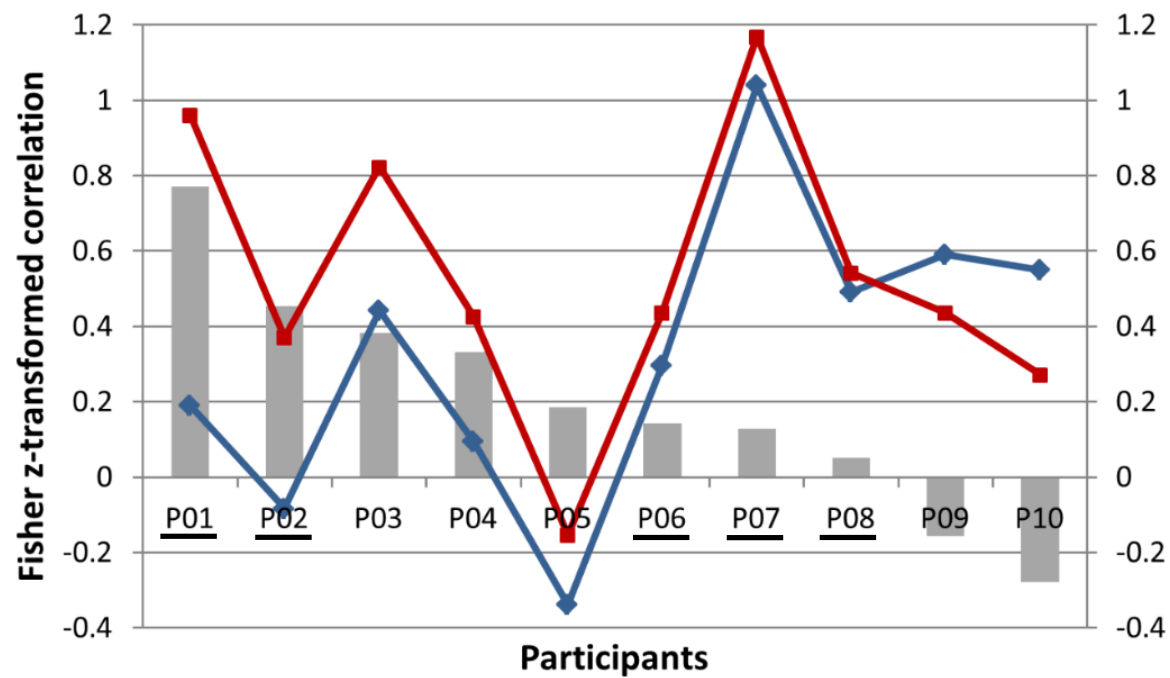

$\Delta$ Feedback - No feedback

$\sim$ No feedback

$\longrightarrow$ Feedback

Figure 5: Comparison of individual gradual self-regulation ability across the two 'type of training' conditions. The figure depicts individual Fisher z-transformed correlation values between obtained single-trial beta values and desired target levels separately for the 'no feedback' (blue line) and 'feedback' (red line) condition and their differences (grey bars). In $80 \%$ of the participants, single-trial beta values were more correlated with the desired target levels when participants received neurofeedback (vs. being not provided with neurofeedback information). Participants with a black underline underwent 'feedback' condition first and 'no feedback' condition second).

\section{Heart and breathing rates}

In Fig. 6, mean heart and breathing rates obtained during the feedback conditions are plotted jointly for P02-P05 and P09 (with all values being in the normal range). While observed differences in heart rate across target level conditions were extremely weak, slightly augmented breathing frequencies were detected for higher target level conditions on a descriptive level. 
A

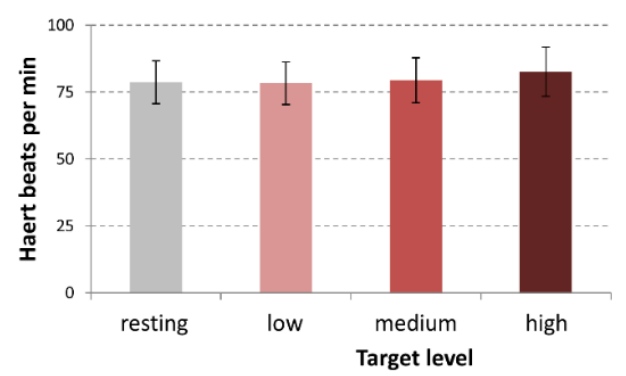

B

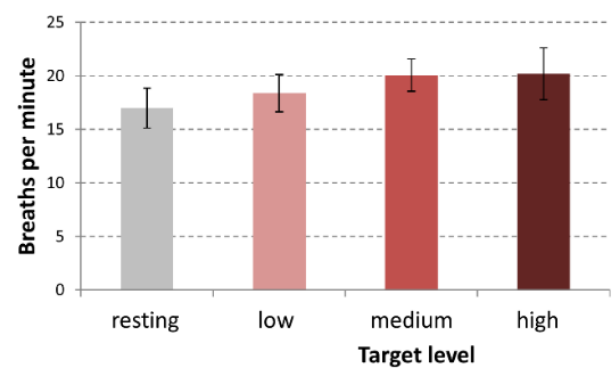

Figure 6: Mean heart and breathing rates for each target level condition. Mean heart (A) and breathing (B) rates of P02-P05 and P09 are plotted separately for each target level condition. While mean heart rates only showed negligible differences across target level conditions, slightly increased breathing frequencies at higher target level conditions can be observed. Error bars indicate variance across participants ( \pm SEM).

\section{DISCUSSION}

The current feasibility study systematically investigated the possibility to gradually modulate the BOLD-signal level within mental task-related regions as well as the potential benefit of providing neurofeedback information in this context. Ten healthy participants underwent two fMRI sessions in which they were asked to reach three different levels of activation by a) applying activation and modulation strategies alone or b) additionally considering neurofeedback about their current brain activation level within a region of interest to optimise their gradual self-regulation performance. The obtained results demonstrate that participants can indeed gradually modulate their brain activation when using appropriate cognitive strategies. Moreover, additionally providing participants with continuous neurofeedback information can further enhance the gradual self-regulation performance.

\section{Self-regulation of regional brain activation to different target levels}

\section{Remarkable gradual self-regulation ability based on suited cognitive strategies}

In the first part of our study, we investigated whether humans are generally (i.e., without getting neurofeedback information) capable of modulating regional brain 
activation gradually (as opposed to an 'all-or-none' strategy employed in previous studies) based on given activation and modulation strategies that emerged from literature and/or intuition of the experimenters (gradual self-regulation effect).

Generally, when looking at the 'no feedback' results, participants succeeded in up-regulating their brain activation level within the activation strategy-related brain region to a great extent (see Fig. $4 \mathrm{~A}$ and $4 \mathrm{C}$ and maximal \% BOLD-signal values in Table 2) and were able to modify their cognitive strategies in such a way that, on the group level, the reached brain activation level significantly increased with each target level step in a linear way (Fig. 4A) and two different target levels could be significantly distinguished (Fig. 4B). The respective single-subject results show that individual participants were well-skilled to gradually self-regulate their brain activation level: $80 \%$ of participants were able to gradually modulate local brain activation to at least two target levels and in $60 \%$, even a significant correlation of the single-trial beta values and the activation target levels could be ascertained (Table 3, Fig. 4A-C).

The remarkable gradual self-regulation performance of our participants might be caused by the following reasons:

1. As established activation strategies were suggested to the participants, they all chose an immediately successful (cognitive) activation strategy (see Table 1) that generally evoked robust regional brain activation (maximal \% BOLD-signal level of the functional localiser data within the region of interest were $>2.0$ in all participants, Table 2). This made it more likely to efficiently apply modulation strategies and to reach intermediate brain activation levels.

2. The information about the potential modulation strategies given to the participants before the MRI session was highly relevant for successfully performing the gradual self-regulation task-indeed resulting in the desired BOLD-signal variations when participants carefully followed these instructions.

3. As half of the participants underwent the 'feedback' condition first and the 'no feedback' condition in a second step, the obtained gradual self-regulation effect might be partially explained by a neurofeedback transfer effect (applying successful modulation strategies refined during 
the earlier 'feedback' condition in the later 'no feedback' condition). However, this seems unlikely as the majority of the participants starting with the 'no feedback' condition demonstrated already a clear gradual modulation ability in the 'no feedback' situation.

Overall, our results are in accordance with previous findings showing that the BOLD-signal level can be modulated temporally by varying certain aspects of mental task performance (e.g., rate of inner speech generation [Shergill et al., 2002], rate of imagined movements [Berman et al., 2012], increased angles of mental rotation [Lipp et al., 2012] or particular cognitive processes as object-based attention [e.g., Culham \& Kanwisher, 2001]).

The applied activation and modulation strategies as well as the selected regions of interest varied considerably across participants, with no clear advantage for any strategy or brain region (see Table 1, Table 2 and Fig. 4C). This implies that gradual self-regulation of brain activation can, in principle, be achieved using various activation and modulation strategies and at (at least) several brain locations.

\section{Enhanced gradual self-regulation ability through neurofeedback}

In a second step of this study, we investigated whether additionally providing neurofeedback information on the current brain activation level can further improve the gradual self-regulation performance (instantaneous feedback effect). The remarkable gradual self-regulation ability already obtained in the 'no feedback' condition (most probably caused by optimal instruction of the participants; see discussion above) made it actually quite challenging to demonstrate an instantaneous feedback effect on top of this gradual self-regulation effect. Despite this, single-trial analysis showed that most participants demonstrated a higher gradual self-regulation performance in the 'feedback' condition compared to the 'no feedback' condition (see increase of correlations of observed single-trial beta values and target levels in Fig. 5 and Table 3) suggesting a benefit of providing neurofeedback on a single-subject level. These single-subject results were jointly analysed on the group level (paired t-test) showing a significant instantaneous feedback effect meaning that providing neurofeedback can indeed further enhance the ability to gradually modulate regional brain activation. Moreover, as can be obtained from Table 3, more significant 'between target level contrasts' (in the 
desired direction) could be differentiated in the 'feedback' condition (vs. in the 'no feedback' condition) in four participants (Table 3 ) and on the group level (see three significant between target level contrasts in the 'feedback' vs. only two significant contrasts in the 'no feedback' condition displayed in Fig. 4B). Finally, using a stricter and more sensitive criterion, namely a significant correlation of the single-trial beta values and the activation target level, actually $80 \%$ of the participants were able to gradually modulate the brain activation level in the 'feedback' condition (vs. $60 \%$ of participants in the 'no feedback' condition; see Table 3). All these results indicate that the additional availability of neurofeedback about the current BOLD-signal level can indeed facilitate the gradual self-regulation performance and therewith confirms our second hypothesis.

The observed instantaneous feedback effect cannot be explained by a trivial training effect (increased gradual self-regulation performance simply caused by repeated mental task performance vs. successful use of neurofeedback information (cf. Sulzer, Sitaram, et al., 2013), as we employed an experimental design that included balancing the 'type of training' conditions across participants. Additionally, the fact that the two participants who showed a lower correlation coefficient in the 'feedback' (vs. the 'no feedback') condition first underwent the 'no feedback' condition, speaks against a simple training effect. Another argument that suggests a real (instantaneous) feedback effect is that all participants starting with the 'feedback' condition performed worse in the following 'no feedback' condition. Note, that the latter clearly points to an instantaneous feedback rather than a feedback transfer effect, i.e., the increased gradual self-regulation ability seems to be bound by the 'feedback' situation and cannot be easily transferred to the 'no feedback' situation.

Though being significant on a group level, the instantaneous feedback effect is admittedly relatively small. Note, however, that if we would not have provided our participants with explicit activation and modulation strategies, this effect would have been most likely larger. We intentionally followed the described procedure (optimal cognitive preparation of the participants) as we aimed at investigating the specific effect that can be attributed solely to the presence of neurofeedback. Note also, that the instantaneous feedback effect had to be established on top of the considerable gradual self-regulation effect (see discussion above). Thus, it was not trivial to demonstrate an instantaneous feedback effect, especially when 
considering that individuals might not immediately benefit from neurofeedback. Processing neurofeedback information (in parallel to mental task execution) strongly increases workload (see results on introspective reports of participants). Thus, gradual self-regulation of the BOLD-signal level might be hampered by the demanding multi-tasking requirements associated with the processing of the neurofeedback (monitoring, interpreting, and accordingly implementing the feedback). This might have been the case for the two participants that were not able to use the neurofeedback information in order to improve their gradual selfregulation performance (P09 and P10; see Fig. 4C, Fig. 5). These participants (both starting with the 'no feedback' condition) probably needed substantially more time to get used to the more demanding (dual-task) feedback situation resulting in a performance drop in the first instance (see Table 3, Fig. 4C, Fig. 5). Note that the amount of neurofeedback training was rather limited in our study (one feedback session including only 30min of neurofeedback training). Participants with initial difficulties, might benefit from extended neurofeedback training across multiple sessions (Frank et al., 2012; Linden et al., 2012; Canterberry et al., 2013; Haller et al., 2013; Hartwell et al., 2013; Li et al., 2013; Ruiz, Lee et al., 2013; Auer et al., 2015; Cordes et al., 2015; Sherwood et al., 2016) or from employing an alternative (individually tailored) neurofeedback display (Krause et al., 2017). Of course, it might be that some individuals will never benefit from neurofeedback or that providing feedback might even work disadvantageously for them. Note, that even if not all individuals benefit from rt-fMRI neurofeedback, it might still constitute a crucial advancement on the individual level. All in all, we conclude that rt-fMRI neurofeedback can enhance the gradual self-regulation ability.

\section{Reflections on task instruction in rt-fMRI neurofeedback studies}

One current debate within the rt-fMRI community concerns the way of instructing participants in neurofeedback experiments. In order to assure a true feedback effect, the practical realization of the feedback and the control condition(s) should solely differ in the presence of the (valid) neurofeedback information - implying that no other crucial difference between conditions should exist. Thus, a feedback effect should not be attributable to, e.g., differences in task instruction but only to the presence of the neurofeedback information (Sulzer, Haller, et al., 2013; Thibault, Lifshitz, \& Raz, 2016). 
Note however, that in case a particular cognitive strategy has been shown to effectively alter brain activation in a wanted direction and this is paralleled with the desired behavioural change, it might be advisable to communicate this cognitive strategy to the participants before the fMRI-neurofeedback session in order to maximise positive effects. However, it might be then questionable whether a positive effect would actually be caused by the neurofeedback training or rather by the successful application of the specific instruction. In a study failing to replicate the pioneering work of DeCharms et al. (2005) on positive rt-fMRI neurofeedback effects in chronic pain, participants were provided with identical task instructions (previously successful mental strategies in the same context) in both the feedback and the control condition and the same pain relief was observed in both conditions (unpublished data, discussed in Sulzer, Haller, et al., 2013). This suggests that the application of a suitable and (to the participants) well-communicated mental strategy can result in the same effects in the feedback and the control condition speaking rather for an effect of the mental strategy and against a true feedback effect (Thibault et al., 2016).

In our current study, participants also received identical instructions concerning potential activation and modulation strategies in both the 'no feedback' and the 'feedback' condition - therewith perfectly matching the two 'type of training' conditions. As these strategies were generally very effective with respect to the purpose of the study (gradual self-regulation), we ascertained a remarkable gradual self-regulation effect already in the absence of neurofeedback. However and most importantly, we were able to demonstrate that participants' performance was increased in the 'feedback' condition which can only be attributed to the additional presence of the neurofeedback information, justifying the interpretation of a true (instantaneous) feedback effect.

Thus, in contrast to the replication study mentioned above we obtained differences between the 'no feedback' and 'feedback' condition - indicating a true (instantaneous) feedback effect next to the gradual self-regulation effect.

\section{Potential for neuroscientific research and clinical applications}

\section{Potential applications for neuroscientific research}

Classical fMRI studies employ the BOLD-signal level as the dependent variable in order to investigate cognitive, sensory, emotional or motor functions of the brain. 
In contrast, fMRI-based neurofeedback allows the use of the brain activation level as the independent variable, allowing for an advanced investigation of brain functions. For example, the specific functional involvement of a particular brain region can be explored by self-regulating its activation and observing accordant behavioural changes (Weiskopf et al., 2003). The current study suggests that it might be possible to implement parametric designs for these purposes, which would constitute a powerful extension. Thus, further rt-fMRI neurofeedback research could investigate whether parametrically varied brain-activation levels are associated with accordant systematic perceptual, cognitive, emotional or behavioural changes.

\section{Potential for clinical application}

\section{BCl-based communication and control}

For brain-based communication and control it is highly desirable to encode a particular intention on the single-trial basis. Previously, we have shown that this is feasible in an rt-fMRI setup using information encoding paradigms combining spatial and temporal BOLD-signal features (Sorger et al., 2009; 2012). In the current study, we investigated the potential of using magnitudinal BOLD-signal features (i.e., different brain-signal levels) for information encoding. When providing participants with appropriate activation and modulation strategies, we obtained medium to high correlations between the desired and the actually achieved brain activation level for the majority of participants already in the 'no feedback' condition. Thus, even without implementing neurofeedback, employing magnitudinal BOLD-signal features might be feasible to neurally encode few information units (like "yes"/"no" or "up"/"down"). Note that this outcome is generally favourable in the $\mathrm{BCl}$ context as it indicates that the suggested novel information encoding approach might qualify for $\mathrm{BCl}$ applications not requiring neurofeedback implementations which are technically much more challenging. Still, our second outcome, namely that the gradual self-regulation ability can be further enhanced by additionally providing neurofeedback information is absolutely desired as the observed gradual self-regulation performance was far from being perfect - especially when looking at the single-trial level. Note however, that averaging $\mathrm{fMRI}$ activation across multiple trials constitutes a powerful option to increase the BOLD-signal's robustness, which has been successfully applied in 
healthy participants (averaging across three trials; Yoo et al., 2004; Lee et al., 2009) and in patients (averaging across five trials; Monti et al., 2010; Naci et al., 2012). Trial averaging, of course, results in a considerably lower information transfer rate (a fortiori taking into account the relatively long information encoding time in fMRI-based $\mathrm{BCls}$ owed to the sluggishness of the hemodynamic brain response). Note however, that the averaging approach might still constitute a valuable option for patients that do not have any other communication and control means left.

Future research might focus on intensive single-case studies (as, for example, performed in Weiskopf et al., 2003) systematically investigating the number of employable BOLD-signal levels starting with only two (extreme) target level conditions and only introducing more target levels when two levels can be sufficiently differentiated (adaptive procedure). In this context, it might be beneficial to start with a neurofeedback training aiming at maximizing the BOLD-signal magnitude in the region of interest as initially reaching higher brain activation levels would most probably increase the ability to (learn to) self-regulate intermediate brain activation levels reliably (increased activation range).

All in all, we think that the suggested approach is promising even if the gradual self-regulation ability might be limited to a few levels. Note, that a differentiation of two BOLD-signal levels on a single-trial basis would already provide a considerable increase in degrees of freedom in hemodynamic $\mathrm{BCl}$ applications, namely when combined with the other employed approaches (e.g., implementing additionally spatial and/or temporal BOLD-signal features for information encoding).

\section{Neurofeedback therapy}

The demonstration of an increased gradual self-regulation ability by means of neurofeedback not only advances $\mathrm{BCl}$ research, but might also extend the current spectrum of neurofeedback therapy paradigms. So far, neurofeedback studies on clinical populations have focused on a maximal up- or down-regulation of regional brain activation as this has been thought to result in a maximal benefit (i.e., a maximal reduction of clinical symptoms).

Note however, that employing a parametric modulation approach in this context might facilitate developing a general understanding of how regional brain activation can be influenced. More particularly, self-regulating brain activation to 
specific target levels might help fine-tuning the applied cognitive strategies in a faster fashion - leading to a steeper learning curve. Moreover, the possibility to reach different activation levels and to gain a more detailed sense for controlling activation in a targeted brain region might enhance the subjective feeling of success and the experience of self-efficacy - being of high importance from a motivational point of view.

Taking all these points together, we think that the parametric modulation approach as introduced in the current study might be a significant asset in the context of neurofeedback therapy. However, this possibility has to be systematically investigated in future studies.

\section{Discussion of potential confounding factors and limitations of the study}

The results of the current study might be confounded by several factors that will be discussed below together with other limitations of the study.

Specific study population: Study participants were all students or staff members of the Faculty of Psychology and Neuroscience at Maastricht University. Thus, most of them had experience in participating in $\mathrm{FMRI}$ experiments. Moreover, they all had specific background knowledge on neuroscience (e.g., with respect to neuroimaging, neurofeedback, $\mathrm{BCl}$ methodology etc.). However, we do not consider this knowledge to account for the ascertained instantaneous feedback effect as neurofeedback learning refers to the practical experience only gained in a neurofeedback situation itself. Moreover, participants could have applied potential pre-knowledge in both experimental conditions ('feedback' and 'no feedback' condition). Thus, we do not think that this pre-knowledge had an effect on the 'feedback' condition only (and thus constituted a confounding factor that could account for the instantaneous feedback effect established in this study). Note however, that relevant methodological pre-knowledge (e.g., in our case, on how to systematically elaborate the best modulation strategy) might constitute a facilitating factor for the neurofeedback learning process and it might be advisable to always provide participants if possible with potentially helpful information. But as this refers to knowledge that cannot be acquired practically in a (preceding) neurofeedback session, it cannot account for the instantaneous feedback effect. 
While our participants had the abovementioned pre-knowledge, they had not participated in a neurofeedback training (with either EEG, fMRI or fNIRS) before. Thus, they had no (in this context critical) practical neurofeedback experience that they could fall back on and could have transferred to the current neurofeedback session.

Limited number of participants: The number of participants in the current study is rather low $(n=10)$. One problem of small sample-sized studies is that the statistical power is low and that the sensitivity to outliers is higher than in studies with large sample sizes. Therefore, the generalization of obtained findings to the population has to be done with care. Note however, that a significant result obtained in a small sample-sized study (when well-controlled for false positives) is even more compelling evidence than the equivalent result with a larger sample-sized study (Friston, 2012). Thus, our results generally support that the gradual modulation approach is feasible in $\mathrm{BCl}$ and neurofeedback contexts and worth to be investigated in more detail and with larger sample sizes.

Not blinding participants: As discussed above, we considered it crucial to keep the information given to the participants before entering the scanner constant across all participants - independently of whether they started with the 'no feedback' or the 'feedback' condition. Therefore, participants were not blinded and we relied on the assumption that our participants tried following the experimenters' instructions to the best of their knowledge and belief.

Choice of control conditions for investigating the instantaneous feedback effect: Several control conditions to investigate an fMRI-neurofeedback effect have been suggested and implemented in the past, e.g., providing no feedback (Auer et al., 2015), sham/pseudo feedback (DeCharms et al., 2004, 2005; Rota et al., 2009; Caria, Sitaram, Veit, Begliomini, \& Birbaumer, 2010; Scharnowski et al., 2012), feedback from another brain region (DeCharms et al., 2005) etc. We chose the 'no feedback' condition for the following reasons: Firstly, this condition was already implemented in our study design as, as a first step, we investigated the principal ability to gradually modulate brain activation (i.e., without providing neurofeedback). Secondly, we considered the 'no feedback' condition as the most 
valid or appropriate control condition in the current $\mathrm{BCl}$ context as for $\mathrm{BCl}$ applications, providing sham/pseudo feedback would not constitute a meaningful option. Note, however, that $\mathrm{BCl}$ applications without involving a neurofeedback component might still constitute a reasonable alternative (see discussion above).

Limited amount of physiological data: Due to technical problems, heart and breathing data were only available for five out of ten participants and merely for the 'feedback' condition (thus for in total $25 \%$ of the fMRI data). Because of the limited amount of physiological-data acquisitions, we were not able to systematically examine the effect of heart and breathing rates on the fMRI signal by adding physiological data as parameter of no interest. This would have constituted a valuable addition.

Cardiorespiratory effects: Analysing available heart and breathing data revealed slightly increased breathing frequencies with higher target level conditions (see Fig. 6B). Physiologically, an increase in the breathing frequency leads to a decrease of the carbon-dioxide ( $\mathrm{CO} 2)$ concentration and an increase of the oxygen (O2) concentration in the blood. Animal experiments systematically varying $\mathrm{O} 2$ concentrations demonstrated that hyperoxia (enhanced levels of $\mathrm{O} 2$ in the blood) actually leads to a decrease of BOLD-signal levels (Sicard \& Duong, 2005; Wibral et al., 2007). In accordance with this, BOLD-signal increases have been reported in hypercapnia (enhanced levels of $\mathrm{CO} 2$ in the blood) (Kastrup, Krüger, Glover, Neumann-Haefelin, \& Moseley, 1999). Both findings indicate that increased breathing frequencies should go along with decreased BOLD-signal levels (thus should rather work against our gradual self-regulation hypothesis).

To summarise, the latter theoretical consideration and our descriptive physiological results imply that the obtained differences in the obtained brain activation magnitude across target level conditions are unlikely to be driven by cardiorespiratory effects.

General arousal effects: Possible changes in general arousal are also unlikely to account for the obtained gradual self-regulation results. Explorative analysis of the fMRI data (results not shown) revealed no widespread activation increases for higher target level conditions that would be expected in that case. 
Study design: We implemented a within-subject design. Actually, a between-subject design would have had certain advantages, especially in terms of avoiding potential feedback transfer effects (e.g., through applying modulation strategies elaborated during earlier 'feedback' runs in subsequent 'no feedback' runs in half of our participants). However, a between-subject design requires a considerably higher number of participants per group than could be realised within the scope of the current study. As the number of participants to be trained in fMRI-based neurofeedback studies is limited in general, a within-subject design was considered more appropriate.

In order to address the discussed limitations and caveats of this study, several follow-up studies are requested. These more extensive studies should involve a considerably higher number of (naïve) participants, implement more fMRI sessions and advanced experimental designs (e.g., alternating between 'no feedback' and 'feedback' runs, involving more trials per participant, testing several visual feedback displays etc.) and include physiological parameters in the data analysis.

\section{CONCLUSIONS}

The current study shows that humans - when being provided with appropriate activation and modulation strategies - are able to modulate the level of regional brain activation as measured with $\mathrm{fMRI}$ gradually. Moreover, we demonstrate that providing participants additionally with neurofeedback on the current BOLD-signal level within the target region can enhance the gradual self-regulation ability. Our findings were observed across a wide variety of activation strategies (mental tasks) and across clinical MR field strengths, indicating that these findings are robust and can be generalised across mental tasks and scanner types. Our study strongly motivates a further exploration of the novel parametric modulation approach that considerably enriches the current spectrum of fMRI-neurofeedback and $\mathrm{BCl}$ methodology which has attracted significant interest in fundamental and clinical neuroscience in the recent past. 


\section{ACKNOWLEDGEMENTS}

We would like to thank Joel Reithler, for his help in analysing the heart and breathing data, Alberto Cassese for statistical advice and Isabelle Bareither for supporting data acquisition. The authors gratefully acknowledge the support of the BrainGain Smart Mix Programme of the Netherlands Ministry of Economic Affairs and the Netherlands Ministry of Education, Culture and Science. This work was also supported by the Netherlands Organization for Scientific Research (NWO; RUBICON 446-09-010 awarded to BS) and the European Research Council (ERC; advanced grant \#269853 awarded to RG). 


\section{REFERENCES}

Auer, T., Schweizer, R., \& Frahm, J. (2015). Training efficiency and transfer success in an extended real-time functional MRI neurofeedback training of the somatomotor cortex of healthy subjects. Frontiers in Human Neuroscience, 9, 547. http://doi.org/10.3389/fnhum.2015.00547

Bardin, J. C., Fins, J. J., Katz, D. I., Hersh, J., Heier, L. A., Tabelow, K., ... Voss, H. U. (2011). Dissociations between behavioural and functional magnetic resonance imaging-based evaluations of cognitive function after brain injury. Brain, 134(3), 769-782. http://doi.org/10.1093/brain/awr005

Berman, B. D., Horovitz, S. G., \& Hallett, M. (2013). Modulation of functionally localized right insular cortex activity using real-time fMRI-based neurofeedback. Frontiers in Human Neuroscience, 7(October), 638. http://doi.org/10.3389/fnhum.2013.00638

Berman, B. D., Horovitz, S. G., Venkataraman, G., \& Hallett, M. (2012). Selfmodulation of primary motor cortex activity with motor and motor imagery tasks using real-time fMRI-based neurofeedback. Neurolmage, 59(2), 917925. http://doi.org/10.1016/j.micinf.2011.07.011.Innate

Birbaumer, N., Ghanayim, N., Hinterberger, T., Iversen, I., Kotchoubey, B., Kübler, A., ... Flor, H. (1999). A spelling device for the paralysed. Nature, 398, 297298. http://doi.org/10.1038/18581

Boly, M., Coleman, M. R., Davis, M. H., Hampshire, A., Bor, D., Moonen, G., ... Owen, A. M. (2007). When thoughts become action: An fMRI paradigm to study volitional brain activity in non-communicative brain injured patients. Neurolmage, 36(3), 979-992. http://doi.org/10.1016/j.neuroimage.2007.02.047

Boynton, G. M., Engel, S. A., Glover, G. H., \& Heeger, D. J. (1996). Linear Systems Analysis of Functional Magnetic Resonance Imaging in Human V1. The Journal of Neuroscience, 16(13), 4207-4221.

Brühl, A. B., Scherpiet, S., Sulzer, J., Stämpfli, P., Seifritz, E., \& Herwig, U. (2014). Real-time Neurofeedback Using Functional MRI Could Improve DownRegulation of Amygdala Activity During Emotional Stimulation: A Proof-ofConcept Study. Brain Topography, 27(1), 138-148. http://doi.org/10.1007/s10548-013-0331-9 
Canterberry, M., Hanlon, C. A., Hartwell, K. J., Li, X., Owens, M., LeMatty, T., ... George, M. S. (2013). Sustained Reduction of Nicotine Craving With RealTime Neurofeedback: Exploring the Role of Severity of Dependence. Nicotine \& Tobacco Research, 15(12), 2120-2024. http://doi.org/10.1093/ntr/ntt122

Caria, A., \& de Falco, S. (2015). Anterior insular cortex regulation in autism spectrum disorders. Frontiers in Behavioral Neuroscience, 9(38), 1-9. http://doi.org/10.3389/fnbeh.2015.00038

Caria, A., Sitaram, R., Veit, R., Begliomini, C., \& Birbaumer, N. (2010). Volitional control of anterior insula activity modulates the response to aversive stimuli. A real-time functional magnetic resonance imaging study. Biological Psychiatry, 68(5), 425-432. http://doi.org/10.1016/j.biopsych.2010.04.020

Caria, A., Veit, R., Sitaram, R., Lotze, M., Weiskopf, N., Grodd, W., \& Birbaumer, N. (2007). Regulation of anterior insular cortex activity using real-time fMRI. Neurolmage, 35 , 1238-1246. http://doi.org/10.1016/j.neuroimage.2007.01.018

Chapin, J. K., Moxon, K. A., Markowitz, R. S., \& Nicolelis, M. A. (1999). Real-time control of a robot arm using simultaneously recorded neurons in the motor cortex. Nature Neuroscience, 2(7), 664-670. http://doi.org/10.1038/10223

Chiew, M., LaConte, S. M., \& Graham, S. J. (2012). Investigation of fMRI neurofeedback of differential primary motor cortex activity using kinesthetic motor imagery. Neurolmage, 61(1), 21-31. http://doi.org/10.1016/j.neuroimage.2012.02.053

Cordes, J. S., Mathiak, K. A., Dyck, M., Alawi, E. M., Gaber, T. J., Zepf, F. D., ... Mathiak, K. (2015). Cognitive and neural strategies during control of the anterior cingulate cortex by fMRI neurofeedback in patients with schizophrenia. Frontiers in Behavioral Neuroscience, 9, 169. http://doi.org/10.3389/fnbeh.2015.00169

Cox, R. W., Jesmanowicz, A., \& Hyde, J. S. (1995). Real-time functional magnetic resonance imaging. Magnetic Resonance Imaging, 33(2), 230-236. http://doi.org/10.1002/mrm.1910330213 
Culham, J. C., \& Kanwisher, N. G. (2001). Neuroimaging of cognitive functions in human parietal cortex. Current Opinion in Neurobiology, 11(2), 157-163. http://doi.org/10.1016/S0959-4388(00)00191-4

DeCharms, R. C., Christoff, K., Glover, G. H., Pauly, J. M., Whitfield, S., \& Gabrieli, J. D. . (2004). Learned regulation of spatially localized brain activation using real-time fMRI. Neurolmage, 21(1), 436-443. http://doi.org/10.1016/j.neuroimage.2003.08.041

DeCharms, R. C., Maeda, F., Glover, G. H., Ludlow, D., Pauly, J. M., Soneji, D., ... Mackey, S. C. (2005). Control over brain activation and pain learned by using real-time functional MRI. Proceedings of the National Academy of Sciences of the United States of America, 102(51), 18626-31. http://doi.org/10.1073/pnas.0505210102

Emmert, K., Breimhorst, M., Bauermann, T., Birklein, F., Van De Ville, D., \& Haller, S. (2014). Comparison of anterior cingulate vs. insular cortex as targets for real-time $\mathrm{fMRI}$ regulation during pain stimulation. Frontiers in Behavioral Neuroscience, 8(350), 1-13. http://doi.org/10.3389/fnbeh.2014.00350

Farwell, L. A., \& Donchin, E. (1988). Talking off the top of your head: toward a mental prosthesis utilizing event-related brain potentials. Electroencephalography and Clinical Neurophysiology, 70(6), 510-523. http://doi.org/10.1016/0013-4694(88)90149-6

Frank, S., Lee, S., Preissl, H., Schultes, B., Birbaumer, N., \& Veit, R. (2012). The Obese Brain Athlete: Self-Regulation of the Anterior Insula in Adiposity. PLoS ONE, 7(8), e42570. http://doi.org/10.1371/journal.pone.0042570

Friston, K. (2012). Ten ironic rules for non-statistical reviewers. Neurolmage, 61(4), 1300-1310. http://doi.org/10.1016/j.neuroimage.2012.04.018

Friston, K. J., Fletcher, P., Josephs, O., Holmes, A., Rugg, M. D., \& Turner, R. (1998). Event-Related fMRI: Characterizing Differential Responses. Neurolmage, 7(1), 30-40. http://doi.org/10.1006/nimg.1997.0306

Garrison, K. A., Santoyo, J. F., Davis, J. H., Thornhill, T. A., Kerr, C. E., \& Brewer, J. A. (2013). Effortless awareness: using real time neurofeedback to investigate correlates of posterior cingulate cortex activity in meditators' self-report. Frontiers in Human Neuroscience, 7(440), 1-9. http://doi.org/10.3389/fnhum.2013.00440 
Garrison, K. A., Scheinost, D., Worhunsky, P. D., Elwafi, H. M., Thornhill, T. A., Thompson, E., ... Brewer, J. A. (2013). Real-time fMRI links subjective experience with brain activity during focused attention. Neurolmage, 81, 110-8. http://doi.org/10.1016/j.neuroimage.2013.05.030

Goebel, R., Sorger, B., Kaiser, J., Birbaumer, N., \& Weiskopf, N. (2004). BOLD brain pong: self-regulation of local brain activity during synchronously scanned, interacting subjects. Abstract viewer/itinerary planner. In Society for Neuroscience, Washington, DC.

Goebel, R., Zilverstand, A., \& Sorger, B. (2010). Real-time fMRI-based braincomputer interfacing for neurofeedback therapy and compensation of lost motor functions. Imaging in Medicine, 2(4), 407-415. http://doi.org/10.2217/iim.10.35

Greer, S. M., Trujillo, A. J., Glover, G. H., \& Knutson, B. (2014). Control of nucleus accumbens activity with neurofeedback. Neurolmage, 96, 237-44. http://doi.org/10.1016/j.neuroimage.2014.03.073

Guan, M., Ma, L., Li, L., Yan, B., Zhao, L., Tong, L., ... Xia, L. (2015). Self-Regulation of Brain Activity in Patients with Postherpetic Neuralgia: A Double-Blind Randomized Study Using Real-Time fMRI Neurofeedback. PLoS ONE, 10(4), e0123675. http://doi.org/10.7910/DVN/27368

Guillot, A., Collet, C., Nguyen, V. A., Malouin, F., Richards, C., \& Doyon, J. (2008). Functional neuroanatomical networks associated with expertise in motor imagery. Neurolmage, $\quad$ 41, http://doi.org/10.1016/j.neuroimage.2008.03.042

Haller, S., Birbaumer, N., \& Veit, R. (2010). Real-time fMRI feedback training may improve chronic tinnitus. European Radiology, 20(3), 696-703. http://doi.org/10.1007/s00330-009-1595-z

Haller, S., Kopel, R., Jhooti, P., Haas, T., Scharnowski, F., Lovblad, K.-O., ... Van De Ville, D. (2013). Dynamic reconfiguration of human brain functional networks through neurofeedback. Neurolmage, 81, 243-52. http://doi.org/10.1016/j.neuroimage.2013.05.019

Hamilton, J. P., Glover, G. H., Bagarinao, E., Chang, C., Mackey, S., Sacchet, M., ... Hamilton, P. (2016). Effects of salience-network-node neurofeedback training on affective biases in major depressive disorder. Psychiatry 
Research:

Neuroimaging,

239,

91-96.

http://doi.org/10.1016/j.pscychresns.2016.01.016

Hampson, M., Stoica, T., Saksa, J., Scheinost, D., Qiu, M., Bhawnani, J., ... Constable, T. (2012). Real-time fMRI Biofeedback Targeting the Orbitofrontal Cortex for Contamination Anxiety. Journal of Visualized Experiments, 59, e3535. http://doi.org/10.3791/3535

Hartwell, K., Hanlon, C., Li, X., Borckardt, J., Canterberry, M., Prisciandaro, J., ... Brady, K. (2016). Individualized real-time fMRI neurofeedback to attenuate craving in nicotine-dependent smokers. Journal of Psychiatry \& Neuroscience, 41(1), 140200.

Hartwell, K. J., Prisciandaro, J. J., Borckardt, J., Li, X., George, M. S., \& Brady, K. T. (2013). Real-time fMRI in the Treatment of Nicotine Dependence: A Conceptual Review and Pilot Studies. Psychology of Addictive Behaviors, 27(2), 501-509. http://doi.org/10.1037/a0028215.Real-time

Hochberg, L. R., Bacher, D., Jarosiewicz, B., Masse, N. Y., Simeral, J. D., Vogel, J., ... Donoghue, J. P. (2012). Reach and grasp by people with tetraplegia using a neurally controlled robotic arm. Nature, 485(7398), 372-375. http://doi.org/10.1038/nature11076

Hochberg, L. R., Serruya, M. D., Friehs, G. M., Mukand, J. A., Saleh, M., Caplan, A. H., ... Donoghue, J. P. (2006). Neuronal ensemble control of prosthetic devices by a human with tetraplegia. Nature, 442, 164-171. http://doi.org/10.1038/nature04970

Karch, S., Keeser, D., Hümmer, S., Paolini, M., Kirsch, V., Karali, T., ... Pogarell, O. (2015). Modulation of Craving Related Brain Responses Using Real-Time fMRI in Patients with Alcohol Use Disorder. PLoS ONE, 10(7), e0133034. http://doi.org/10.1371/journal.pone.0133034

Kastrup, A., Krüger, G., Glover, G. H., Neumann-Haefelin, T., \& Moseley, M. E. (1999). Regional Variability of Cerebral Blood Oxygenation Response to Hypercapnia. Neurolmage, 10, 675-681. http://doi.org/10.1006/nimg.1999.0505

Kim, D.-Y., Yoo, S.-S., Tegethoff, M., Meinlschmidt, G., \& Lee, J.-H. (2015). The Inclusion of Functional Connectivity Information into fMRI-based Neurofeedback Improves Its Efficacy in the Reduction of Cigarette Cravings. 
Journal of Cognitive Neuroscience, 27(8), 1552-1572. http://doi.org/10.1162/jocn

Kirsch, M., Gruber, I., Ruf, M., Kiefer, F., \& Kirsch, P. (2015). Real-time functional magnetic resonance imaging neurofeedback can reduce striatal cuereactivity to alcohol stimuli. Addiction Biology, 1-11. http://doi.org/10.1111/adb.12278

Koush, Y., Rosa, M. J., Robineau, F., Heinen, K., W Rieger, S., Weiskopf, N., ... Scharnowski, F. (2013). Connectivity-based neurofeedback: Dynamic causal modeling for real-time fMRI. Neurolmage, 81, 422-430. http://doi.org/10.1016/j.neuroimage.2013.05.010

Kübler, A., Kotchoubey, B., Hinterberger, T., Ghanayim, N., Perelmouter, J., Schauer, M., ... Birbaumer, N. (1999). The thought translation device: a neurophysiological approach to communication in total motor paralysis. Experimental Brain Research, 124(2), 223-232. http://doi.org/10.1007/s002210050617

Krause, F., Benjamins, C., Lührs, M., Eck, J., Noirhomme, Q., Rosenke, M., ..., Goebel, R. (2017). Real-time fMRI-based self-regulation of brain activation across different visual feedback presentations. Brain-Computer Interfaces, 4(1-2), 87-101. http://doi.org/10.1080/2326263X.2017.1307096

LaConte, S. M., Peltier, S. J., \& Hu, X. P. (2007). Real-Time fMRI Using Brain-State Classification. Human Brain Mapping, 28(10), 1033-1044. http://doi.org/10.1002/hbm.20326

Lee, J.-H., Ryu, J., Jolesz, F. A., Cho, Z.-H., \& Yoo, S.-S. (2009). Brain-machine interface via real-time fMRI: Preliminary study on thought-controlled robotic arm. Neuroscience Letters, 450(1), 1-6. http://doi.org/10.1016/j.neulet.2008.11.024

Leuthardt, E. C., Schalk, G., Wolpaw, J. R., Ojemann, J. G., \& Moran, D. W. (2004). A brain-computer interface using electrocorticographic signals in humans. Journal of Neural Engineering, 1(2), 63-71. http://doi.org/10.1088/1741$2560 / 1 / 2 / 001$

Li, X., Hartwell, K. J., Borckardt, J., Prisciandaro, J. J., Saladin, M. E., Morgan, P. S., ... George, M. S. (2013). Volitional Reduction of Anterior Cingulate Cortex Activity Produces Decreased Cue Craving in Smoking Cessation: A 
Preliminary Real-Time fMRI Study. Addiction Biology, 18(4), 739-748. http://doi.org/10.1111/j.1369-1600.2012.00449.x.Volitional

Linden, D. E. J., Habes, I., Johnston, S. J., Linden, S., Tatineni, R., Subramanian, L., ... Goebel, R. (2012). Real-time self-regulation of emotion networks in patients with depression. PloS One, 7(6), e38115. http://doi.org/10.1371/journal.pone.0038115

Lipp, I., Benedek, M., Fink, A., Koschutnig, K., Reishofer, G., Bergner, S., ... Neubauer, A. (2012). Investigating Neural Efficiency in the Visuo-Spatial Domain: An fMRI Study. PLoS ONE, 7(12), e51316. http://doi.org/10.1371/journal.pone.0051316

Maclnnes, J. J., Dickerson, K. C., Chen, N.-K., \& Adcock, R. A. (2016). Cognitive Neurostimulation: Learning to Volitionally Sustain Ventral Tegmental Area Activation. Neuron, 89(6),

1331-1342. http://doi.org/10.1016/j.neuron.2016.02.002

Mellinger, J., Schalk, G., Braun, C., Preissl, H., Rosenstiel, W., Birbaumer, N., \& Kübler, A. (2007). An MEG-based Brain-Computer Interface (BCl). Neurolmage, 36(3), 581-593. http://doi.org/10.1016/j.micinf.2011.07.011.Innate

Monti, M. M., Vanhaudenhuyse, A., Coleman, M. R., Boly, M., Pickard, J. D., Tshibanda, L., ... Laureys, S. (2010). Willful Modulation of Brain Activity in Disorders of Consciousness. The New England Journal of Medicine, 362(7), 579-589.

Naci, L., Monti, M. M., Cruse, D., Kübler, A., Sorger, B., Goebel, R., ... Owen, A. M. (2012). Brain-computer interfaces for communication with nonresponsive patients. Annals of Neurology, 72, 312-323. http://doi.org/10.1002/ana.23656

Nijboer, F., Sellers, E. W., Mellinger, J., Jordan, M. a, Matuz, T., Furdea, A., ... Kübler, A. (2008). A P300-based brain-computer interface for people with amyotrophic lateral sclerosis. Clinical Neurophysiology, 119(8), 1909-1916. http://doi.org/10.1016/j.clinph.2008.03.034

Ogawa, S., Lee, T. M., Kay, A. R., \& Tank, D. W. (1990). Brain magnetic resonance imaging with contrast dependent on blood oxygenation. Proceedings of the 
National Academy of Sciences of the United States of America, 87(24), 9868-72.

Owen, A. M., Coleman, M. R., Boly, M., Davis, M. H., Laureys, S., \& Pickard, J. D. (2006). Detecting Awareness in the Vegetative State. Science, 313, 1402. http://doi.org/10.1126/science.1130197

Ramsey, N. F., van De Heuvel, M. P., Kho, K. H., \& Leijten, F. S. S. (2006). Towards Human BCl Applications Based on Cognitive Brain Systems: An Investigation of Neural Signals Recorded From the Dorsolateral Prefrontal Cortex. Transactions on Neural Systems and Rehabilitation Engineering, 14(2), 214-217. http://doi.org/10.1109/TNSRE.2006.875582

Robineau, F., Rieger, S. W., Mermoud, C., Pichon, S., Koush, Y., Van De Ville, D., ... Scharnowski, F. (2014). Self-regulation of inter-hemispheric visual cortex balance through real-time fMRI neurofeedback training. Neurolmage, 100, 1-14. http://doi.org/10.1016/j.neuroimage.2014.05.072

Rota, G., Sitaram, R., Veit, R., Erb, M., Weiskopf, N., Dogil, G., \& Birbaumer, N. (2009). Self-Regulation of Regional Cortical Activity Using Real-Time fMRI: The Right Inferior Frontal Gyrus and Linguistic Processing. Human Brain Mapping, 30(5), 1605-14. http://doi.org/10.1002/hbm.20621

Ruiz, S., Birbaumer, N., \& Sitaram, R. (2013). Abnormal neural connectivity in schizophrenia and fMRI-brain-computer interface as a potential therapeutic approach. Frontiers in Psychiatry, 4(March), 17. http://doi.org/10.3389/fpsyt.2013.00017

Ruiz, S., Lee, S., Soekadar, S. R., Caria, A., Veit, R., Kircher, T., ... Sitaram, R. (2013). Acquired Self-control of Insula Cortex Modulates Emotion Recognition and Brain Network Connectivity in Schizophrenia. Human Brain Mapping, 34(1), 200-212. http://doi.org/10.1002/hbm.21427

Scharnowski, F., Hutton, C., Josephs, O., Weiskopf, N., \& Rees, G. (2012). Improving Visual Perception through Neurofeedback. The Journal of Neuroscience, 32(49), 17830-41. http://doi.org/10.1523/JNEUROSCI.6334-11.2012

Scheinost, D., Stoica, T., Saksa, J., Papademetris, X., Constable, R. T., Pittenger, C., \& Hampson, M. (2013). Orbitofrontal cortex neurofeedback produces lasting changes in contamination anxiety and resting-state connectivity. Translational Psychiatry, 3, e250. http://doi.org/10.1038/tp.2013.24 
Scherer, R., Müller, G. R., Neuper, C., Graimann, B., \& Pfurtscheller, G. (2004). An Asynchronously Controlled EEG-Based Virtual Keyboard: Improvement of the Spelling Rate. IEEE Transactions on Biomedical Engineering, 51(6), 979984. http://doi.org/10.1109/TBME.2004.827062

Shergill, S. S., Brammer, M. J., Fukuda, R., Bullmore, E., Amaro, E., Murray, R. M., \& McGuire, P. K. (2002). Modulation of activity in temporal cortex during generation of inner speech. Human Brain Mapping, 16(4), 219-227. http://doi.org/10.1002/hbm.10046

Sherwood, M. S., Kane, J. H., Weisend, M. P., \& Parker, J. G. (2016). Enhanced control of dorsolateral prefrontal cortex neurophysiology with real-time functional magnetic resonance imaging (rt-fMRI) neurofeedback training and working memory practice. Neurolmage, 124, 214-223. http://doi.org/10.1016/J.NEUROIMAGE.2015.08.074

Sicard, K. M., \& Duong, T. Q. (2005). Effects of hypoxia, hyperoxia, and hypercapnia on baseline and stimulus-evoked BOLD, CBF, and CMRO2 in spontaneously breathing animals. Neurolmage, 25(3), 850-858. http://doi.org/10.1016/j.micinf.2011.07.011.Innate

Sorger, B., Dahmen, B., Reithler, J., Gosseries, O., Maudoux, A., Laureys, S., \& Goebel, R. (2009). Another kind of 'BOLD Response': answering multiplechoice questions via online decoded single-trial brain signals. Progress in Brain Research, 177, 275-292. http://doi.org/10.1016/S00796123(09)17719-1

Sorger, B., Reithler, J., Dahmen, B., \& Goebel, R. (2012). A Real-Time fMRI-Based Spelling Device Immediately Enabling Robust Motor-Independent Communication. Current Biology, 22(14), 1333-1338. http://doi.org/10.1016/j.cub.2012.05.022

Subramanian, L., Hindle, J. V, Johnston, S., Roberts, M. V, Husain, M., Goebel, R., \& Linden, D. (2011). Real-time functional magnetic resonance imaging neurofeedback for treatment of Parkinson's disease. The Journal of Neuroscience : The Official Journal of the Society for Neuroscience, 31(45), 16309-17. http://doi.org/10.1523/JNEUROSCI.3498-11.2011

Sulzer, J., Haller, S., Scharnowski, F., Weiskopf, N., Birbaumer, N., Blefari, M. L., ... Sitaram, R. (2013). Real-time fMRI neurofeedback: Progress and challenges. 
Neurolmage,

76,

386-399.

http://doi.org/10.1016/J.NEUROIMAGE.2013.03.033

Sulzer, J., Sitaram, R., Laura, M., Kollias, S., Birbaumer, N., Enno, K., ... Gassert, R. (2013). Neurofeedback-mediated self-regulation of the dopaminergic midbrain. Neurolmage, 83, 817-825. http://doi.org/10.1016/j.neuroimage.2013.05.115

Thibault, R. T., Lifshitz, M., \& Raz, A. (2016). The self-regulating brain and neurofeedback: Experimental science and clinical promise. Cortex, 74, 247261. http://doi.org/10.1016/J.CORTEX.2015.10.024

Weiskopf, N., Klose, U., Birbaumer, N., \& Mathiak, K. (2005). Single-shot compensation of image distortions and BOLD contrast optimization using multi-echo EPI for real-time fMRI. Neurolmage, 24(4), 1068-79. http://doi.org/10.1016/j.neuroimage.2004.10.012

Weiskopf, N., Mathiak, K., Bock, S. W., Scharnowski, F., Veit, R., Grodd, W., ... Birbaumer, N. (2004). Principles of a brain-computer interface (BCl) based on real-time functional magnetic resonance imaging (fMRI). IEEE Transactions on Biomedical Engineering, 51(6), 966-70. http://doi.org/10.1109/TBME.2004.827063

Weiskopf, N., Scharnowski, F., Veit, R., Goebel, R., Birbaumer, N., \& Mathiak, K. (2004). Self-regulation of local brain activity using real-time functional magnetic resonance imaging (fMRI). Journal of Physiology-Paris, 98(4-6), 357-373. http://doi.org/10.1016/j.jphysparis.2005.09.019

Weiskopf, N., Veit, R., Erb, M., Mathiak, K., Grodd, W., Goebel, R., \& Birbaumer, N. (2003). Physiological self-regulation of regional brain activity using realtime functional magnetic resonance imaging (fMRI): methodology and exemplary data. Neurolmage, 19(3), 577-86.

Wibral, M., Muckli, L., Melnikovic, K., Scheller, B., Alink, A., Singer, W., \& Munk, M. $H$. (2007). Time-dependent effects of hyperoxia on the BOLD fMRI signal in primate visual cortex and LGN. Neurolmage, 35(3), 1044-1063. http://doi.org/10.1016/j.neuroimage.2006.12.039

Yoo, J. J., Hinds, O., Ofen, N., Thompson, T. W., Whitfield-Gabrieli, S., Triantafyllou, C., \& Gabrieli, J. D. E. (2012). When the brain is prepared to learn: 
enhancing human learning using real-time fMRI. Neurolmage, 59(1), 84652. http://doi.org/10.1016/j.neuroimage.2011.07.063

Yoo, S.-S., Fairneny, T., Chen, N.-K., Choo, S.-E., Panych, L. P., Park, H., ... Jolesz, F. A. (2004). Brain-computer interface using fMRI: spatial navigation by thoughts. NeuroReport, 15(10), 1591-1595. http://doi.org/10.1097/01.wnr.0000133296.39160.fe

Yoo, S.-S., \& Jolesz, F. A. (2002). Functional MRI for neurofeedback: feasibility study on a hand motor task. NeuroReport, 13(11), 1377-1381. http://doi.org/10.1097/00001756-200208070-00005

Young, B. M., Nigogosyan, Z., Walton, L. M., Song, J., Nair, V. A., Grogan, S. W., ... Prabhakaran, V. (2014). Changes in functional brain organization and behavioral correlations after rehabilitative therapy using a brain-computer interface. Frontiers in Neuroengineering, 7(26), 15. http://doi.org/10.3389/fneng.2014.00026

Young, K. D., Zotev, V., Phillips, R., Misaki, M., Yuan, H., Drevets, W. C., \& Bodurka, J. (2014). Real-Time fMRI Neurofeedback Training of Amygdala Activity in Patients with Major Depressive Disorder. PLOS ONE, 9(2), e88785. http://doi.org/10.1371/journal.pone.0088785

Zhang, G., Yao, L., Zhang, H., Long, Z., \& Zhao, X. (2013). Improved working memory performance through self-regulation of dorsal lateral prefrontal cortex activation using real-time fMRI. PloS One, 8(8), e73735. http://doi.org/10.1371/journal.pone.0073735

Zhang, G., Zhang, H., Li, X., Zhao, X., Yao, L., \& Long, Z. (2013). Functional Alteration of the DMN by Learned Regulation of the PCC Using Real-Time fMRI. IEEE Transactions on Neural Systems and Rehabilitation Engineering, 21(4), 595-606. http://doi.org/10.1109/TNSRE.2012.2221480

Zilverstand, A., Sorger, B., Sarkheil, P., \& Goebel, R. (2015). fMRI neurofeedback facilitates anxiety regulation in females with spider phobia. Frontiers in Behavioral Neuroscience, 9(148), $1-12$. http://doi.org/10.3389/fnbeh.2015.00148

Zotev, V., Krueger, F., Phillips, R., Alvarez, R. P., Simmons, W. K., Bellgowan, P., ... Bodurka, J. (2011). Self-Regulation of Amygdala Activation Using Real-Time 
fMRI Neurofeedback. PLoS ONE, 6(9), e24522. http://doi.org/10.1371/journal.pone.0024522

Zotev, V., Yuan, H., Misaki, M., Phillips, R., Young, K. D., Feldner, M. T., \& Bodurka, J. (2016). Correlation between amygdala BOLD activity and frontal EEG asymmetry during real-time $\mathrm{fMRI}$ neurofeedback training in patients with depression. Neurolmage: Clinical, 11, 224-238. http://doi.org/10.1016/j.nicl.2016.02.003 



\section{CHAPTER 3}

THE PRE-STIMULUS DEFAULT MODE NETWORK STATE PREDICTS COGNITIVE TASK PERFORMANCE LEVELS ON A MENTAL ROTATION TASK
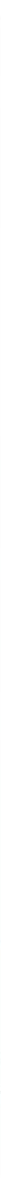


\section{Based on:}

Kamp, T., Sorger, B., Benjamins, C., Hausfeld, L., \& Goebel, R. (2018). The prestimulus default mode network state predicts cognitive task performance levels on a mental rotation task. Brain and Behavior, 8(8), e01034. DOI: 10.1002/brb3.1034 


\begin{abstract}
Linking individual task performance to preceding, regional brain activation is an ongoing goal of neuroscientific research. Recently, it could be shown that the activation and connectivity within large-scale brain networks prior to task onset influence performance levels. More specifically, pre-stimulus default mode network (DMN) effects have been linked to performance levels in sensory near-threshold tasks, as well as cognitive tasks. However, it still remains uncertain how the DMN state preceding cognitive tasks affects performance levels when the period between task trials is long and flexible, allowing participants to engage in different cognitive states. Therefore, we here investigated whether the pre-stimulus activation and within-network connectivity of the DMN are predictive of the correctness and speed of task performance levels on a cognitive (match-to-sample) mental rotation task, employing a sparse event-related functional magnetic resonance imaging ( $\mathrm{fMRI}$ ) design. We found that pre-stimulus activation in the DMN predicted the speed of correct trials, with a higher amplitude preceding correct fast response trials compared to correct slow response trials. Moreover, we found higher connectivity within the DMN before incorrect trials compared to correct trials. These results indicate that pre-existing activation and connectivity states within the DMN influence task performance on cognitive tasks, both effecting the correctness and speed of task execution. The findings support existing theories and empirical work on relating mind-wandering and cognitive task performance to the DMN and expand these by establishing a relationship between the pre-stimulus DMN state and the speed of cognitive task performance.
\end{abstract}




\section{INTRODUCTION}

In the past, neuroscientific research has aimed at identifying factors contributing to performance variations on diverse tasks and highlighted the role of pre-stimulus activation and connectivity in brain regions specific to the task at hand (Colas \& Hsieh, 2014; Giesbrecht, Weissman, Woldorff, \& Mangun, 2006; Hesselmann, Sadaghiani, Friston, \& Kleinschmidt, 2010; Ploner, Lee, Wiech, Bingel, \& Tracey, 2010; Sapir, D’Avossa, McAvoy, Shulman, \& Corbetta, 2005; Weissman, Roberts, Visscher, \& Woldorff, 2006). This evidence for a baseline "preparedness" of task-related brain regions was soon extended to a more global dimension by uncovering that large-scale networks constitute different baseline states contributing to different task-performance levels. More specifically, it has been proposed that spontaneous fluctuations in these networks during the period prior to task onset influence task performance to various degrees, introducing the idea that specific network states affect subsequent performance levels (Colas \& Hsieh, 2014; Li, Yan, Bergquist, \& Sinha, 2007; Mayhew, Ostwald, Porcaro, \& Bagshaw, 2013; Rahnev, Bahdo, de Lange, \& Lau, 2012; Sadaghiani, Hesselmann, \& Kleinschmidt, 2009; Sadaghiani, Poline, Kleinschmidt, \& D’Esposito, 2015; Soravia et al., 2016; Vanhaudenhuyse et al., 2011).

One line of research focused on differentiating an intrinsic and an extrinsic network, both contributing to different levels of awareness (Fox et al., 2005; Fransson, 2005; Tian et al., 2007; Vanhaudenhuyse et al., 2011). While the extrinsic network has been associated with the awareness of external stimuli in the environment, and is fuelled by sensory information, the intrinsic network can be channelled by internally generated mental processes, possibly independent of sensory input (Fox et al., 2005; Lieberman, 2007; Vanhaudenhuyse et al., 2011). Brain regions involved in this intrinsic network have been proposed to comprise regions of the so-called default-mode network (DMN) containing hubs in the posterior cingulate cortex (PCC)/precuneus, medial prefrontal cortex (MPFC)/ventral anterior cingulate cortex (VACC) and parietal regions. Cognitive processes driven by these regions involve self-generated thought, autobiographical memory, mind-wandering, and daydreaming (Lieberman, 2007; Mason et al., 2007; Scheibner, Bogler, Gleich, Haynes, \& Bermpohl, 2017; Smallwood \& Schooler, 2015). When these brain regions are active, the mind is therefore involved in internally-directed thoughts and external tasks might be difficult to perform when initiated unexpectedly. In 
fact, it has been proposed that pre-stimulus activity in the DMN can be linked to subsequent task performance. It was shown that pre-stimulus DMN activity could predict somatosensory perception (Boly et al., 2007; Mayhew et al., 2013) and auditory stimulus detection (Sadaghiani et al., 2009). More specifically, in studies on pre-stimulus effects of the DMN on somatosensory and pain perception, Boly et al. (2007) showed that a higher activation in DMN-related regions before the application of a sensory (thermal) stimulus predicted the conscious perception of the stimulus, compared to no conscious perception. Conversely, there is other evidence for a higher pre-stimulus activation in the DMN preceding a more intense perception of painful thermal stimulation (Mayhew et al., 2013). In agreement to this study, auditory stimulus detection was shown to be facilitated when the DMN activation was enhanced before stimulus presentation (Sadaghiani et al., 2009).

The DMN has also been linked to cognitive task performance levels, in particular to selective and sustained attention by looking at attentional lapses and response errors. In one early study, it was shown that attentional lapses were associated with a higher task-induced DMN activation on a selective attention task (Weissman et al., 2006). Furthermore, it has been shown that a higher pre-stimulus DMN activation precedes errors on a go/no-go task (Li et al., 2007), as well as errors in a Flanker task (Eichele et al., 2008), compared to correct task performance. Since these early studies, pre-stimulus DMN effects have been investigated more extensively, showing a more differential role of these effects on task performance. For instance, a higher pre-stimulus DMN activation was related to a state of attentional stability in a spatial attention task, while a reduced activation was related to a more flexible ability to reallocate attention (Sali, Courtney, \& Yantis, 2016). Extending these results, it has been proposed that there are two attentional states which modify cognitive performance levels (Esterman, Noonan, Rosenberg, \& DeGutis, 2013), one more stable and less error-prone state, and one state which leads to suboptimal sustained task performance. Interestingly, the more stable and less error-prone state was generally characterised by a high DMN activity. At the same time, the chance of errors became more likely when the DMN activation rose beyond a moderate level. Recently, one study has also looked into pre-stimulus DMN effects on performance in an emotional memory task. In this study, Soravia et al. (2016) showed that a lower DMN activation before the initial encoding of emotional pictures is beneficial for subsequent recognition performance, even one 
week later. In this research, participants were presented with emotional (positive and negative) and neutral pictures and were subsequently tested on the recognition of these pictures. When the DMN activation was comparatively low prior to stimulus presentation, participants were more likely to recognise the pictures in a memory retrieval test one week later suggesting that when internally-directed mental processes are suppressed, cognitive task processes are facilitated.

The abovementioned studies within the cognitive task domain either used cues in order to indicate an approaching trial (Li et al., 2007; Sali et al., 2016), or employed event-related designs with relatively short inter-stimulus periods (Eichele et al., 2008, Esterman et al., 2013; Soravia et al., 2016; Weissman et al., 2006). Thus, participants were externally cued and thereby triggered into a "task state" (for cued paradigms) or had little time to switch this cognitive state (for paradigms with short inter-trial times). How the pre-stimulus DMN state relates to cognitive task performance in a setting which gives participants room to engage in different cognitive states therefore still remains unclear. Therefore, we employed an uncued, sparse event-related design to investigate potential pre-stimulus DMN effects on cognitive task performance using the match-to-sample mental rotation task, which is a well-studied classical cognitive task (Shepard \& Metzler, 1971). We hypothesised that a lower DMN activation and within-network functional connectivity would be beneficial for task performance, as in this case, the mind would be more involved in externally-related thoughts, compared to a higher activation of the DMN during internally-directed thoughts. We used a match-tosample mental rotation task so that we were able to present short, individual trials. At the same time, trials could be individually adapted, ensuring a constant difficulty level across participants.

Thus, exploring cognitive task performance levels on the grounds of the DMN's state preceding a match-to-sample mental rotation task, the current study aimed at investigating

1. whether the pre-stimulus activation and/or connectivity within the DMN can dissociate the correctness of task performance (correct vs. incorrect responses), and 
2. whether the pre-stimulus activation and/or connectivity within the DMN can predict the speed of task execution within the correct responses (fast vs. slow trials).

\section{MATERIALS AND METHODS}

\section{Participants}

Fourteen healthy volunteers (mean age: $26 \pm 2.9$ SD years, seven female, two lefthanded), with normal or corrected-to-normal vision participated in the study. Participants were all students or staff members of the Faculty of Psychology and Neuroscience at Maastricht University, gave written informed consent before the experimental sessions and were monetarily compensated for their participation. The experimental procedure was approved by the Ethics Review Committee Psychology and Neuroscience at Maastricht University.

\section{Experimental design}

Participants attended two experimental sessions on two days, one behavioural pre-testing and one fMRI scanning session.

Behavioural pre-testing and cognitive task

During the 20 min pre-testing, volunteers performed a match-to-sample mental rotation task (Shepard \& Metzler, 1971). This pre-testing was used to ensure that participants were able to perform the task, as well as identifying the individual difficulty level (angle rotation) for keeping performance across participants identical (aiming at $70 \%$ correct trials). Participants were visually presented with two images of three-dimensional (3D) objects on a black background (Fig. 1) taken from a mental rotation stimulus library (Peters \& Battista, 2008). In each trial, the two objects were either rotated, but identical shapes (same object), or rotated and mirrored shapes (different object) of one another and were presented for 1.5s, followed by a baseline period varying per trial between 2.5 and 4.5s (Fig. 1). Participants were asked to indicate whether the two objects were the same (just rotated) or different (additionally mirrored) shapes by button presses as fast and as accurately as possible. As participants previously showed a variation in cognitive 
ability on mental rotation tasks and the difficulty level can be manipulated based on increasing the angle rotation between the two objects (Peters \& Battista, 2008; Shepard \& Metzler, 1971), the angle rotation at which each individual participant scored $71 \%$ correct on average was identified based on a staircase procedure. The staircase procedure was a simple two-up, one-down procedure, increasing the angle rotation difference between the two objects by $10^{\circ}$ each time the participants responded correctly two times in a row, and lowering the angle rotation difference by $10^{\circ}$ when one incorrect response was made.

\section{A Behavioral pre-testing}

B $\mathrm{fMRI}$
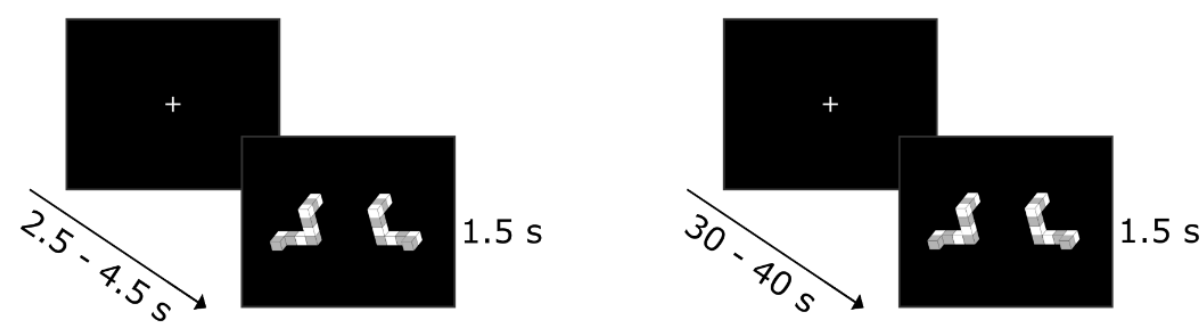

Staircase procedure,

4 - 5 runs, settling at $70 \%$ correct

Figure 1: Match-to-sample mental rotation task. Participants underwent one behavioural pre-testing session, assessing their individual angle rotation at which they scored $70 \%$ correct responses on a match-to-sample mental rotation task. Trials were presented for $1.5 \mathrm{~s}$, interleaved with a jittered baseline of 2.5 to $4.5 \mathrm{~s}(\mathrm{~A})$. In the subsequent fMRI session, four to five runs of the match-to-sample mental rotation task were performed in a sparse event-related design. 22 trials were presented, interleaved with long baseline periods, jittered between 30 and 40s (B).

\section{fMRI session}

After informing participants about the experimental procedure, anatomical images were acquired, followed by a $15 \mathrm{~min}$ resting state run in order to identify individual DMNs. During the resting state run, participants were asked to keep their eyes open, fixating a fixation cross and to not think of anything in particular. Four to five runs of the match-to-sample mental rotation task were administered consecutively to investigate pre-stimulus effects of the DMN state on performance levels. Different from the pre-testing session, trials were presented in a sparse 
event-related design, with inter-stimulus intervals ranging between 30 and 40s, so that each run consisted of 22 trials in total (resulting in a run length of around 14 $\min 30 \mathrm{~s}$ ). These long baseline periods were chosen so that participants could freely engage in different cognitive states. Moreover, they ensured that the blood oxygenation level dependent (BOLD) response went back to baseline and eliminated any carry-over effects from the previous trials. During the baseline periods, participants were asked to focus on a fixation cross and to not think of anything in particular. The first trial of each participant used the angle rotation between the two objects at which they scored $70 \%$ correct at the end of the staircase procedure of the pre-testing session. This was done to ensure that participants were able to do the task, but at the same time ensuring that there were a sufficient amount of incorrect trials for subsequent analyses.

\section{Data acquisition}

Anatomical and functional MRI data were acquired using a $3 T$ whole-body MAGNETOM PrismaFit scanner (Siemens AG, Erlangen, Germany). Participants were comfortably placed in the scanner and their heads were comfortably padded with foam to avoid head motion. T1-weighted anatomical scans were obtained for each participant using a three-dimensional magnetization-prepared rapid-acquisition gradient-echo (MPRAGE) sequence $(192$ slices, slice thickness $=1 \mathrm{~mm}$, no gap, $\mathrm{TR}=2250 \mathrm{~ms}, \mathrm{TE}=2.21 \mathrm{~ms}, \mathrm{FA}=9^{\circ}, \mathrm{FOV}=256 \times 256 \mathrm{~mm} 2$, matrix size $=256 \times 256$, total scan time $=8 \mathrm{~min} 26 \mathrm{~s}$ ). Functional images were acquired using multiband (MB) accelerated echo-planar imaging (EPI) (Moeller et al., 2010), obtaining one resting state run (900 volumes, 48 slices, voxel dimensions $=2.5 \mathrm{~mm}$ isotropic, no gap, $\mathrm{TR}=1000 \mathrm{~ms}, \mathrm{TE}=31 \mathrm{~ms}, \mathrm{FA}=62^{\circ}, \mathrm{FOV}=224 \times 224 \mathrm{~mm} 2$, matrix size $=89.6 \times 89.6, \mathrm{MB}$ factor $=4$, slice order $=$ interleaved) and four to five mental rotation task runs (equal scanning parameters, 880 volumes).

\section{Data analysis}

\section{Behavioural data}

The responses of each participant were sorted post hoc in two different ways: 
1) CORRECTNESS OF TASK PERFORMANCE: In order to investigate whether correct trials could be distinguished from incorrect trials based on the pre-stimulus DMN state, trials were sorted accordingly. The percentage of correct responses for each individual participant was calculated and assessed in terms of suitability for further analysis. As a result, participants were excluded from this analysis when scoring at around chance level (below 60\% correct on average) or when having too little trials with an incorrect response (scoring above $90 \%$ correct).

2) SPEED OF CORRECT TASK PERFORMANCE: To determine whether the pre-stimulus DMN state could dissociate different levels of correct task performance, correct response trials were sorted into fast and slow trials. As the angle rotations varied across participants (due to the staircase procedure and individual performance on the task), a median split of the reaction times was performed for each angle rotation per participant.

Trials with a response time longer than $1.5 \mathrm{~s}$ after stimulus offset, as well as trials with missing responses were excluded from further analyses.

\section{Pre-processing of imaging data}

All pre-processing and analyses of the imaging data were performed with BrainVoyager 20.4 (BrainInnovation, Maastricht, the Netherlands). Anatomical images were corrected for spatial intensity inhomogeneity and subsequently normalised into $\mathrm{MNI}$ (Montreal Neurological Institute) stereotactic space. Functional data were pre-processed using 3D motion correction, slice scan time correction, linear trend removal and a temporal high-pass filter (cut-off value: $0.008 \mathrm{~Hz})$.

\section{Individual DMN definition}

The individual DMNs were identified based on the data from the resting state run using the following steps:

1) Two individual seed-based analyses were performed for each participant using seeds of $10 \mathrm{~mm}$ spheres in two main nodes of the DMN, the posterior cingulate cortex $(P C C) /$ precuneus $(x=-5, y=-49, z=40)$ and the medial 
prefrontal cortex (MPFC)/ventral anterior cingulate cortex ( $\mathrm{VACC})(\mathrm{x}=-1, \mathrm{y}$ $=-47, z=4)$. The respective coordinates were selected based on a meta-analyses about these hubs analysed from resting state data (Fox et al., 2005). For these seed-based analyses, a general linear model (GLM) was calculated, including the time course of the PCC/precuneus or MPFC/vACC as the main predictor, as well as the realignment parameters (three rotations and three translations), the signal from the white matter, and the signal from the ventricles as confounding predictors. The connectivity maps were consecutively corrected for multiple comparisons using cluster-size thresholding (with an initial threshold of $\mathrm{p}<0.001$ ) and the individual DMN was defined as the overlap of the resulting two maps.

2) A group DMN mask was created by applying the abovementioned steps on the group level, performing a random effects GLM analysis across data from all participants. The individual DMNs were subsequently masked with the group result.

3) In order to concentrate on the areas of the $\mathrm{DMN}$ which showed a negative task-induced activation and exclude extrinsically-related areas (e.g., visual cortex), we further constrained the networks with the task-negative network of each participant using the mental rotation task runs (GLM analysis, contrast mental rotation vs. baseline, $p<0.001$, uncorrected).

\section{Pre-stimulus DMN activation}

In order to assess differences in pre-stimulus activation patterns within the whole DMN across task performance levels, a random effects group GLM was performed within the individual DMNs. The GLM included predictors for correct and incorrect responses (or fast and slow trials) and four stick predictors covering the peri-stimulus period from -2 to $+1 \mathrm{~s}$. A paired-samples $t$-test was carried out for correct vs. incorrect responses (fast trials vs. slow trials) at time point 0 (stimulus onset). To further investigate whether either of the two DMN hub regions drives potential pre-stimulus effects, we carried out the same analysis based on the $\mathrm{PCC} /$ precuneus and the MPFC/vACC alone (with individual hubs defined as under the following section 'Pre-stimulus within-DMN connectivity').

Additionally, a linear contrast analysis was performed across the four stick predictors within the whole DMN in order to test the propagation of the DMN 
effects over time while approaching a correct vs. an incorrect trial (fast vs. slow trial). A linear contrast analysis tests for a hypothesised linear pattern in the data. For this, the incorrect (slow) beta values for each time point entering the analysis $(t=-2,-1,0,+1)$ were subtracted from the corresponding correct (fast) beta value. A linear contrast was defined by assigning contrast coefficients $(-3,-1,+1,+3)$ to the resulting difference values. This contrast was subsequently tested against 0 to investigate a linear pattern $(\alpha=.05)$.

This peri-stimulus time window was chosen for the linear contrast analysis as previous research used time points just prior, at stimulus onset or one time point after stimulus onset (as the hemodynamic delay does not cause an effect of stimulus onset on the BOLD response at this time point, compare Hesselmann et al., 2010) to investigate pre-stimulus effects (e.g., Coste et al., 2011; Esterman et al., 2013; Giesbrecht et al., 2006; Hesselmann et al., 2010; Mayhew et al., 2013; Sadaghiani et al., 2009; Soravia et al., 2016). Hereby, the effects can be reliably disentangled from the previous trial and can be interpreted in light of the momentary DMN state just before task onset.

\section{Pre-stimulus within-DMN connectivity}

A beta series correlation analysis (Rissman, Gazzaley, \& D'Esposito, 2004) was implemented to investigate the differences in task performance with regard to the functional connectivity prior task onset between the two main hubs of the DMN (PCC/precuneus and MPFC/vACC) in a region-of-interest approach. The analysis was done by applying the following steps:

1) The $P C C /$ precuneus and the MPFC/vACC were identified in each hemisphere as $4 \mathrm{~mm}$ spheres surrounding the lowest activation point during the mental rotation task for each participant, masked with the individually defined DMN (resulting in four regions of interest). As a prerequisite, the chosen voxels for these two main hubs needed to be correlated significantly.

2) A GLM was calculated including predictors for the task performance conditions (correct vs. incorrect responses or fast vs. slow trials) and a finite impulse response (FIR) model with stick predictors for the pre-stimulus period -2 to 0 s. 
3) Single-trial beta values on the basis of the GLM analysis were extracted for the pre-stimulus period for all four regions and averaged across hemispheres for PCC/precuneus and MPFC/VACC, respectively.

4) The resulting single-trial betas between PCC/precuneus and MPFC/vACC hubs were correlated for correct and incorrect responses (fast trials and slow trials) separately.

5) A paired-samples Wilcoxon signed-rank test was performed on the Fisher $z$-transformed correlation coefficients in order to test the difference between correct vs. incorrect responses (fast trials vs. slow trials) ( $\alpha=.05)$.

\section{RESULTS}

\section{Behavioural results}

Participants performed the mental rotation task during the MRI session with an average percentage of correct responses of $76.5 \%$ ( \pm 11.2 STD). One participant was excluded from further analyses because he did not exceed chance level performance ( $47.73 \%$ correct responses). Thus, data of 13 participants were used for the fast vs. slow trial analyses. As two participants had too few incorrect responses in order to compare correct vs. incorrect trials (4.1\% and $9.1 \%)$, data of eleven participants were included for the correct vs. incorrect response analyses.

\section{Individual DMN definition}

For each participant, an individual DMN could be identified based on the resting state run and the objective procedure described above. Fig. 2 shows the group average of the individual networks (Fig. 2A) and the probabilistic map of all individual DMNs (Fig. 2B). 
A
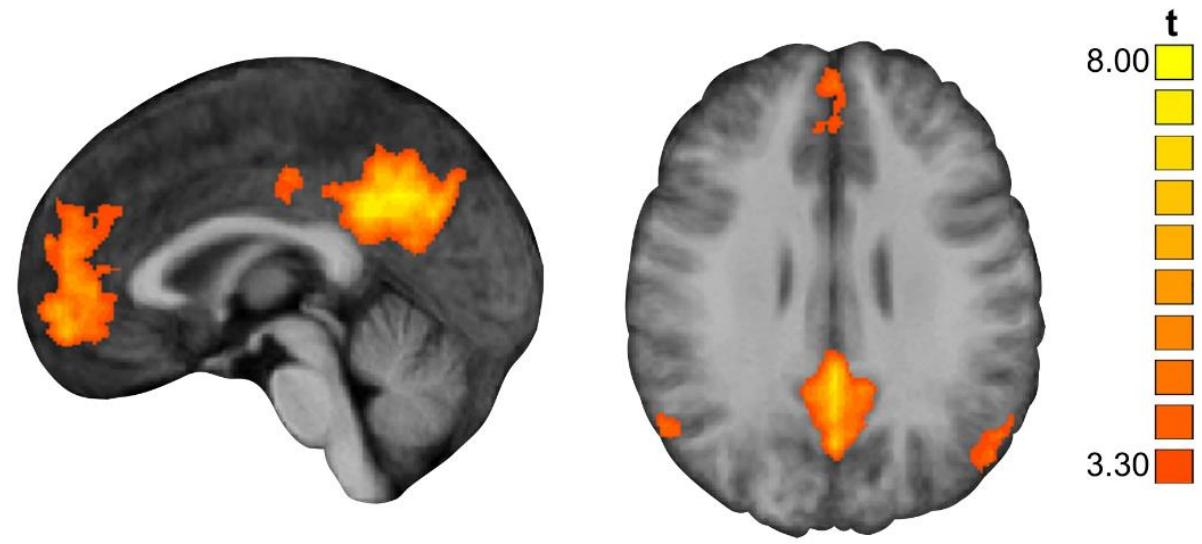

B
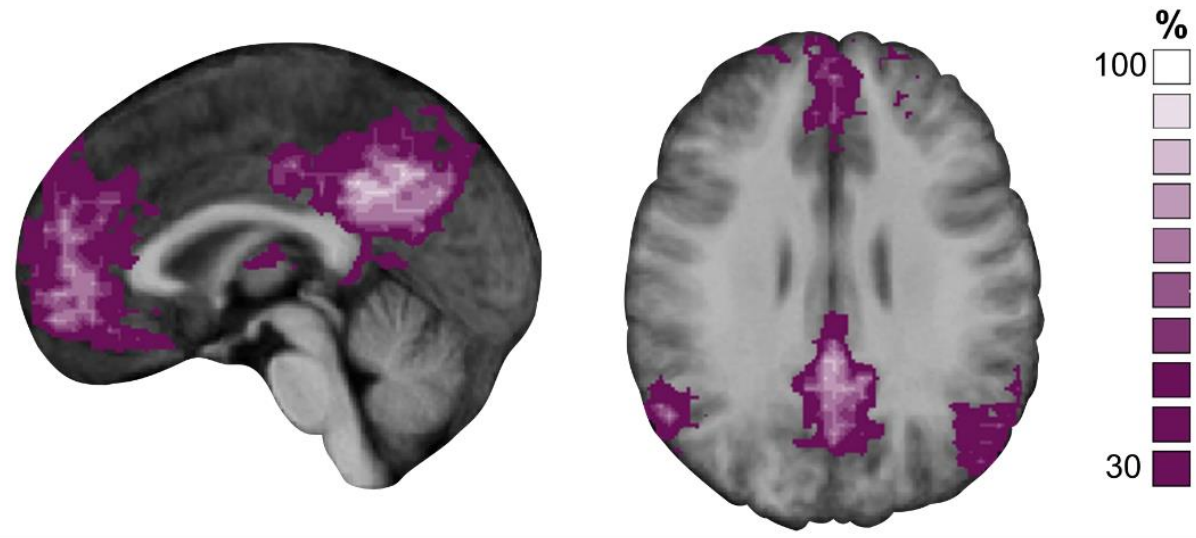

Figure 2: Result of DMN definition. A. Group average of the DMN obtained from the resting state random effects analysis of all participants included in the fast vs. slow trial analysis (cluster-size thresholded at $p<0.001$ ) overlaid on slices of the participants' mean anatomy in MNI space. B. Probabilistic map of individual DMN definitions showing the percentage of spatial overlap of the individual DMN definitions across participants.

Remarks: Left panels: sagittal view, right panel: transversal view, $\mathrm{MNI}$ coordinates: $\mathrm{x}=0, \mathrm{z}=28$.

\section{Pre-stimulus DMN activation}

The pre-stimulus DMN activation did not dissociate between correct and incorrect responses as there was no difference in the activation of the $D M N$ at stimulus onset (Fig. 3A, $t[10]=0.63, p=0.27$, paired-samples t-test, one-sided). Moreover, neither the pre-stimulus PCC/precuneus, nor the MPFC/vACC activation could differentiate 
correct from incorrect responses (PCC/precuneus: $t[10]=-0.231, p=0.41$, paired-samples t-test, one-sided; MPFC/vACC: $t[10]=-0.333, p=0.37$ ). Additionally, the related linear contrast analysis did not yield significance $(F[1,10]=0.89, p=0.368)$. However, the activation in the individual DMNs prior to stimulus presentation was predictive of correct task performance levels, varying between fast and slow trials. Before fast trials, the DMN showed a reduced activation in comparison to slow trials (Fig. 3B, t[12] $=-2.36$, df $=12, p=0.018$, paired-samples t-test, one-sided). Furthermore, both hub regions of the $\mathrm{PCC} /$ precuneus and MPFC/VACC separately showed a significantly lower pre-stimulus activation for fast compared to slow trials (PCC/precuneus: $\mathrm{t}[12]=-1.947, \quad \mathrm{p}=0.038$, paired-samples $\mathrm{t}$-test, one-sided; MPFC/vACC: $t[12]=-1.856, p=0.044$, paired-samples $t$-test, one-sided). Moreover, linear contrast analysis showed that for the fast vs. slow analysis, there was a significantly increasing difference in the activation of the DMN between fast and slow trials the closer the time point was to stimulus presentation $(F[1,12]=6.22, p=0.028)$.

\section{Pre-stimulus within-DMN connectivity}

Differences of within-DMN connectivity states predicted correct and incorrect responses with a lower connectivity between PCC/precuneus and MPFC/VACC preceding correct trials than incorrect trials (Fig. 4A, Wilcoxon signed rank test, $Z=-2.67, p<0.008$ ). However, the same did not hold for the fast vs. slow trial analysis, as no connectivity differences between the two trial types could be observed within the DMN prior task onset (Fig. 4B, Wilcoxon signed rank test, $Z=-0,094, p=0.925)$. 
A

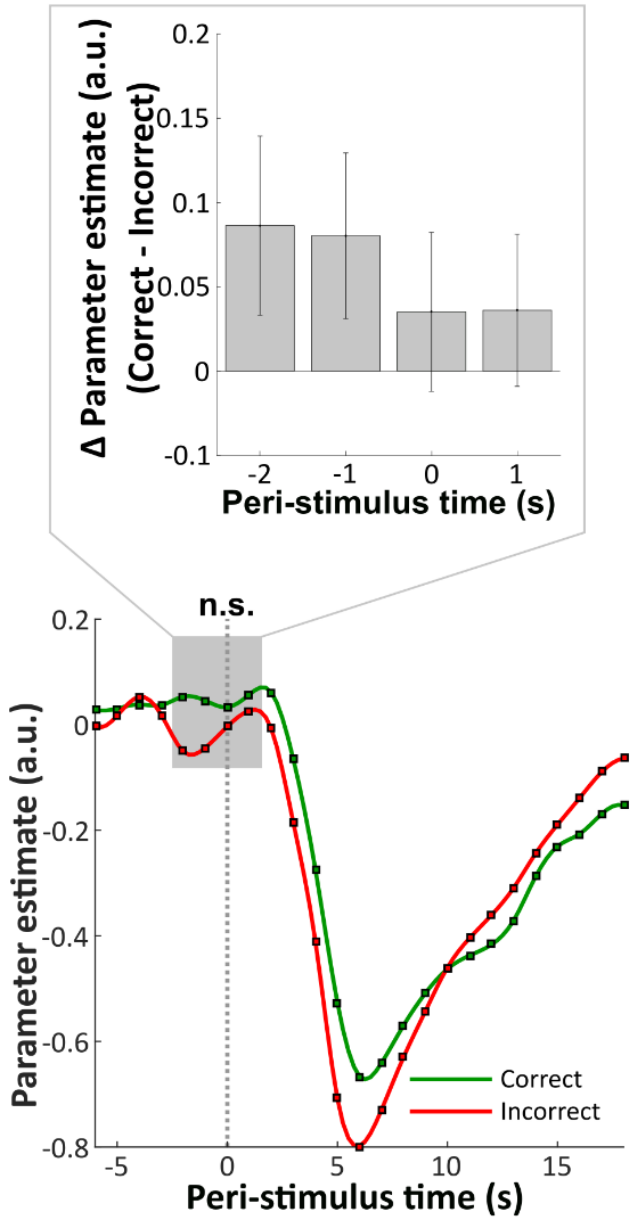

B
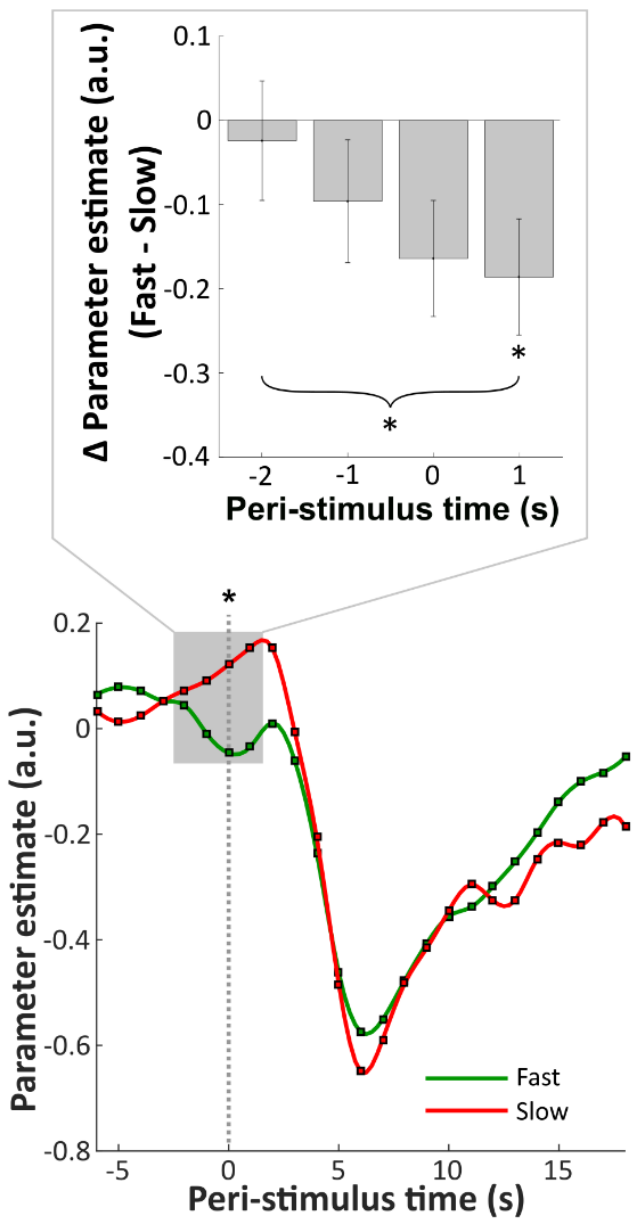

Figure 3: Pre-stimulus DMN activation for both correctness and speed of task performance analyses. A. Upper panel: The difference of obtained peri-stimulus beta values and standard error, comparing correct vs. incorrect responses in the DMN within the tested time window (peri-stimulus time points $-2 \mathrm{~s}$ until $+1 \mathrm{~s})$. Pre-stimulus activation at time point 0 was not significant $(\mathrm{t}[10]=0.63, \mathrm{p}=0.27$, paired-samples t-test, one-sided). Also the linear contrast analysis across the tested time window did not reach significance $(F[1,10]=0.89, p=0.368)$. Lower panel: Peri-stimulus beta time series averaged across all individual DMNs surrounding stimulus onset (indicated by grey dashed line) for correct (green curve) and incorrect (red curve) trials. The grey rectangle depicts the time window used for testing. The $x$-axis represents the peri-stimulus time period (in s), while the $y$-axis depicts the parameter estimates in arbitrary units (beta values). B. Upper panel: The difference of obtained peri-stimulus beta values and standard error, comparing fast vs. slow trials in the DMN within the tested time window (peri-stimulus time points $-2 \mathrm{~s}$ until $+1 \mathrm{~s}$ ). Pre-stimulus activation at time point 0 was significant $(\mathrm{t}[12]=-2.36, \mathrm{df}=12, \mathrm{p}=0.018$, paired-samples $\mathrm{t}$-test, one-sided) with $\mathrm{a} \rightarrow$ 
higher activation in the DMN prior slow trials, indicated by the asterisk. Furthermore, the linear contrast analysis showed a significant linear decrease of the difference of fast-slow trials $(F[1,12]=6.22, p=0.028$, depicted by curly bracket). Lower panel: Peri-stimulus beta time series averaged across all individual DMNs surrounding stimulus onset (indicated by grey dashed line) for fast (green curve) and slow (red curve) trials. The grey rectangle depicts the time window used for testing.

Remark: $\Delta=$ difference.

A

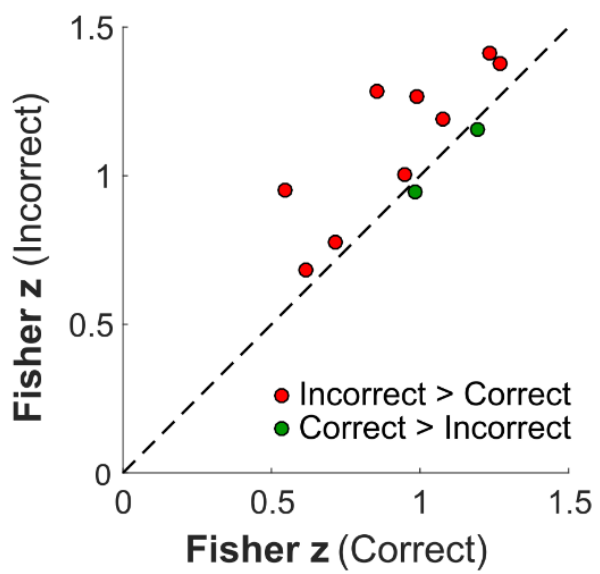

B

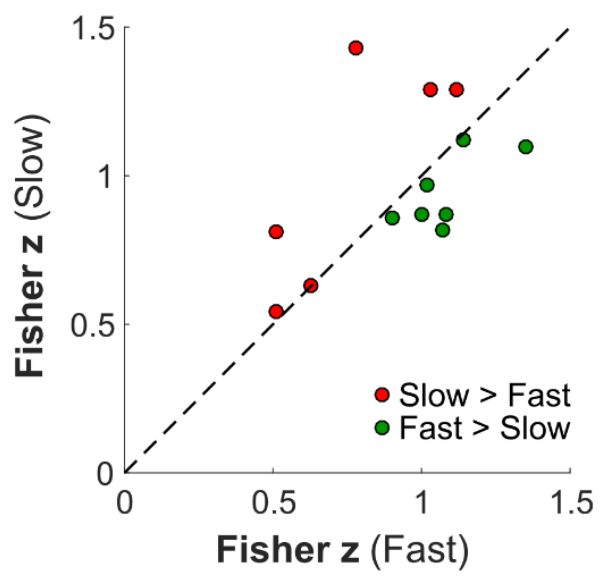

Figure 4: Pre-stimulus within-DMN connectivity for both correctness and speed of task performance analyses. Results of the beta series correlation of main hubs of the DMN for the correctness of task performance (A) and speed of task performance analyses (B). Each dot represents the two Fisher z-transformed correlation coefficients between PCC/precuneus and MPFC/vACC for the pre-stimulus period of the respective analysis for an individual participant, i.e. dots falling above the dashed line represent participants with a higher DMN-connectivity for incorrect (slow) trials (red dots), whereas dots below the dashed line represent participants who showed a higher within-DMN connectivity for correct (fast) trials (green dots). The Wilcoxon signed rank test of correct vs. incorrect responses was significant $(p<0.008)$, with a higher DMN-connectivity prior to incorrect vs. correct trials.

\section{DISCUSSION}

The aim of the present study was to investigate whether the DMN state prior to task onset is predictive of cognitive task performance levels on a mental rotation task (note that the word predictive is here and in the following meant in a correlational, not causal sense). To do this, we employed a sparse event-related fMRI design, evaluating activation and connectivity levels within the DMN prior to 
the onset of a match-to-sample mental rotation task with regard to task performance levels. The study revealed two major relationships between the pre-stimulus DMN state and task performance. First, the amplitude of pre-stimulus DMN activation is associated with correct task performance levels, being able to predict the speed of task performance. The whole-network activation analysis showed that before fast, correct trials, the DMN activation was lower than before slow correct trials (Fig. 3). Furthermore, the difference between the fast and slow pre-stimulus DMN activation became more pronounced until trial onset (Fig. 3B, linear contrast analysis). Second, the within-DMN connectivity preceding trial onset could predict the correctness of task performance. The connectivity analysis showed that before correct task execution, the two major DMN hubs $\mathrm{PCC} /$ precuneus and MPFC/vACC were correlated less than before incorrect task performance (Fig. 4A). Taken together, these complementary results for pre-stimulus activation and connectivity effects on cognitive task performance suggest that both types of analysis are sensitive for different task effects. Therefore, future studies should look on both types of analysis in order to better understand the DMN's role in cognition.

The results of this study are in line with empirical work, as well as theoretical considerations on the DMN network function and cognitive task performance. Previous research investigating the role of the DMN preceding task performance supports our findings that a lower pre-stimulus DMN state is beneficial for cognitive task performance (Eichele et al., 2008; Esterman et al., 2013; Li et al., 2007; Sali et al., 2016; Soravia et al., 2016; Weissman et al., 2006). In the current study, participants performed the mental rotation task in a sparse event-related design and were thus not presented with visual input for a longer period. Thereby, they were flexible to engage in different cognitive modes. A similar sparse event-related design as employed in the current study measured DMN activation before a Stroop task (Coste, Sadaghiani, Friston, \& Kleinschmidt, 2011). While the authors did not find pre-stimulus DMN effects, results indicated a trend for a decreased activation in DMN-related regions for faster trials on this cognitive task compared to slower trials. Thus, our findings extend prior research by showing that in a cognitive task with a low frequency of task trials, cognitive performance is enhanced when participants are engaged in a state of relatively low DMN activation just prior to a task trial. Furthermore, we show that not only a decreased activation level, but 
also a decreased connectivity within the DMN can lead to better task performance on a cognitive task. This supports the idea that fluctuations in the DMN might be meaningful for subsequent task performance, establishing dynamic states and herewith contributing to differential task outcomes. However, previous studies suggest that the nature of the relationship between the $\mathrm{DMN}$ and task performance has not been uniquely established. While the abovementioned studies show converging evidence for a beneficial role of a reduced DMN state before cognitive task onset, a number of other studies have recently shown a more variable role of the DMN for sensory tasks (Boly et al., 2007; Mayhew et al., 2013; Sadaghiani et al., 2009). These studies employed near-threshold sensory tasks, on the one hand showing a lower pre-stimulus activation or connectivity predicted enhanced levels in detection performance (Boly et al., 2007), on the other hand showing a higher pre-stimulus $\mathrm{DMN}$ activation for more intense perception (e.g., more sensitive auditory stimulus detection, more extreme perception during thermal stimulation) of sensory stimuli (Mayhew et al., 2013; Sadaghiani et al., 2009; Sadaghiani et al., 2015). However, the tasks at hand relied primarily on processing in sensory areas, rather than higher-order cognitive areas. The DMN might therefore play different roles with regard to task performance when integrating sensory and higher-order cognitive processes. In contrast to sensory tasks, cognitive tasks such as the match-to-sample mental rotation task employed in the current study might increase the need to distribute connections across different modules instead of relying on network integrity (Sadaghiani et al., 2015). It might be possible that within diverse DMN states, the brain benefits from a less integrated DMN (manifested in less functional connectivity within the network) in order to respond optimally to a changing environment. It is therefore conceivable that the difference found between the abovementioned studies and the current study might be caused by the task at hand (sensory vs. cognitive tasks). In order to reconcile the variable findings within the sensory task domain and to relate pre-stimulus DMN effects in both sensory and cognitive tasks, a future study could employ stimuli which permit both a cognitive and a sensory task for the participant. Such a study would have the power to investigate the $\mathrm{DMN}^{\prime} \mathrm{s}$ role in both sensory and cognitive task performance, while keeping sensory input constant.

The decreased DMN activation and connectivity between PCC/precuneus and MPFC/vACC preceding task onset can be interpreted in light of the literature on 
mind-wandering and its relationship to the $\mathrm{DMN}$. In recent years, it has been shown that levels of DMN activation are linked to the frequency and depth of mind-wandering (Christoff, Gordon, Smallwood, Smith, \& Schooler, 2009; Mason et al., 2007; Scheibner et al., 2017; Smallwood \& Schooler, 2015). Hereby, mind-wandering was defined as any kind of cognition independent of the task at hand, involving processes like retrieval of autobiographic memory, future planning or evaluating and judging the present (Scheibner et al., 2017). Mind-wandering has been associated with several impairments in cognitive functioning (for a review, see Smallwood \& Schooler, 2015), however, a reduction of mind-wandering, as well as concurrent decrease of the DMN could be observed when applying meditation strategies (Berkovich-Ohana, Harel, Hahamy, Arieli, \& Malach, 2016; Scheibner et al., 2017). Furthermore, these behavioural and neural changes in the $D M N$ were associated with an increase in performance on working memory tasks (Berkovich-Ohana et al., 2016). The current project supports this idea by showing that potential mind-wandering episodes (as represented by a heightened DMN activation/connectivity in the baseline period just before task onset) might lead to decreased task performance. Furthermore, spontaneous fluctuations of task modes have been shown to occur at around 20s, and fluctuations of external vs. internal awareness were correlated with DMN activation variability (Vanhaudenhuyse et al., 2011). In the current study, introducing long inter-stimulus intervals of 30-40s with no task-related stimulation in an otherwise stimulus-deprived environment of the MRI scanner made it possible for participants to switch task modes, potentially shifting from task states to mind-wandering phases. This suggests that there may be a direct link between mind-wandering, activation in the DMN and behavioural performance on cognitive tasks, opening up potential scenarios for training cognition and performance levels by, for example, meditation practices. However, it is not clear whether participants were mind-wandering more actively in the slow or incorrect trials compared to the fast or correct trials. Therefore, introducing thought probes after the individual trials in order to measure mind-wandering or task-related thoughts should be introduced in future studies in order to make direct inferences about mind-wandering preceding task onset and poor task performance.

Another consideration with regard to the current study concerns the analysis of incorrect trials. Pre-stimulus activation in the DMN could dissociate fast from slow 
correct trials, however, did not predict whether a trial was performed correctly or incorrectly (Fig. 3A). While in correct trials, performance can be evaluated on the basis of the cognitive processes taking place (by looking at differential task performance in the realm of reaction times), this is not possible with regard to the incorrect trials, where we do not have the possibility to draw conclusions about the cognitive processes at hand. Moreover, the current study looked at pre-stimulus DMN effects on correctness of task performance as well as speed of correct task execution, which is why the study design used a staircase procedure to keep the difficulty level at around $70 \%$ correct responses, ensuring that also incorrect trials could occur. Due to this, two restrictions were induced: the amount of potential correct task responses was limited and the angle difference between the two objects varied across trials for each participant (making the reaction times dependent on the angle difference in each trial, as there is a positive association between angle difference and reaction times (Shepard \& Metzler, 1971)). In order to increase the power and potentially fine-grain the analysis on different clusters of reaction times, future studies should employ a cognitive task which makes reaction times comparable across trials or should focus the design on correct trials and keep the angle difference between the objects constant. By doing this, reaction times could be used as a regressor in the analysis instead of transforming them into categories of fast and slow trials. In this case, it would be possible to investigate whether the pre-stimulus DMN state has an even more elaborate prediction strength of differential task outcome, by being able to differentiate across a continuum of responses.

In conclusion, the current study emphasises the role of the pre-stimulus DMN state in task performance, by showing that the activation and within-network connectivity of the DMN can predict the response speed and the correctness, respectively. These results provide further evidence for large-scale network influences on cognitive behaviour and underlines the importance of investigating pre-stimulus activation and connectivity effects within the DMN in a variety of tasks. 


\section{ACKNOWLEDGEMENTS}

This work was supported by the Human Enhancement and Learning (HEaL) grant for Maastricht University, the Netherlands Organization for Scientific Research (NWO; VENI grant 451-17-033 to L.H.). We would like to thank Armin Heinecke for his valuable support during data analysis. 


\section{REFERENCES}

Berkovich-Ohana, A., Harel, M., Hahamy, A., Arieli, A., \& Malach, R. (2016). Alterations in task-induced activity and resting-state fluctuations in visual and DMN areas revealed in long-term meditators. Neurolmage, 135, 125134. doi:10.1016/j.neuroimage.2016.04.024

Boly, M., Balteau, E., Schnakers, C., Degueldre, C., Moonen, G., Luxen, A., ... Laureys, S. (2007). Baseline brain activity fluctuations predict somatosensory perception in humans. Proceedings of the National Academy of Sciences, 104(29), 12187-12192.

Christoff, K., Gordon, A. M., Smallwood, J., Smith, R., \& Schooler, J. W. (2009). Experience sampling during $\mathrm{fMRI}$ reveals default network and executive system contributions to mind wandering. Proceedings of the National Academy of Sciences, 106(21), 8719-8724. doi: 10.1073/pnas.0900234106

Colas, J. T., \& Hsieh, P. J. (2014). Pre-existing brain states predict aesthetic judgments. Human Brain Mapping, 35(7), 2924-2934. doi: $10.1002 / \mathrm{hbm} .22374$

Coste, C. P., Sadaghiani, S., Friston, K. J., \& Kleinschmidt, A. (2011). Ongoing brain activity fluctuations directly account for intertrial and indirectly for intersubject variability in stroop task performance. Cerebral Cortex, 21(11), 2612-2619. doi: 10.1093/cercor/bhr050

Eichele, T., Debener, S., Calhoun, V.D., Specht, K., Engel, A.K., Hugdahl, K., von Cramon, D.Y., \& Ullsperger, M. (2008). Prediction of human errors by maladaptive changes in event-related brain networks. Proceedings of the National Academy of Sciences, 105(16), 6173-6178. doi: 10.1073/pnas. 0708965105

Esterman, M., Noonan, S.K., Rosenberg, M., \& DeGutis, J. (2013). In the zone or zoning out? Tracking behavioral and neural fluctuations during sustained attention. Cerebral Cortex, 23(11), 2712-2723. doi: 10.1093/cercor/bhs261

Fox, M. D., Snyder, A. Z., Vincent, J. L., Corbetta, M., Essen, D. C. Van, \& Raichle, M. E. (2005). The human brain is intrinsically organized into dynamic, anticorrelated functional networks. Proceedings of the National Academy of Sciences, 102(27), 9673-9678. 
Fransson, P. (2005). Spontaneous low-frequency BOLD signal fluctuations: An fMRI investigation of the resting-state default mode of brain function hypothesis. Human Brain Mapping, 26(1), 15-29. doi: 10.1002/hbm.20113

Giesbrecht, B., Weissman, D. H., Woldorff, M. G., \& Mangun, G. R. (2006). Pretarget activity in visual cortex predicts behavioral performance on spatial and feature attention tasks. Brain Research, 1080(1), 63-72. doi: 10.1016/j.brainres.2005.09.068

Hesselmann, G., Sadaghiani, S., Friston, K. J., \& Kleinschmidt, A. (2010). Predictive coding or evidence accumulation? False inference and neuronal fluctuations. PLoS ONE, 5(3), 1-5. doi: 10.1371/journal.pone.0009926

Li, C.-S. R., Yan, P., Bergquist, K. L., \& Sinha, R. (2007). Greater activation of the "default" brain regions predicts stop signal errors. Neurolmage, 38(3), 640648. doi: 10.1016/j.neuroimage.2007.07.021

Lieberman, M. D. (2007). Social cognitive neuroscience: A review of core processes. Annual Review of Psychology, 58, 259-289. doi: 10.1146/annurev.psych.58.110405.085654

Mason, M. F., Norton, M. I., van Horn, J. D., Wegner, D. M., Grafton, S. T., \& Macrae, C. N. (2007). Wandering minds: The default mode network and stimulusindependent thought. Science, 315(5810), 393-395.

Mayhew, S. D., Ostwald, D., Porcaro, C., \& Bagshaw, A. P. (2013). Spontaneous EEG alpha oscillation interacts with positive and negative BOLD responses in the visual-auditory cortices and default-mode network. Neurolmage, 76, 362372. doi: 10.1016/j.neuroimage.2013.02.070

Moeller, S., Yacoub, E., Olman, C. A., Auerbach, E., Strupp, J., Harel, N., Ugurbil, K. (2010). Multiband multislice GE-EPI at 7 T, with 16-fold acceleration using partial parallel imaging with application to high spatial and temporal wholebrain fMRI. Magnetic Resonance in Medicine, 63(5), 1144-1153. doi: 10.1002/mrm.22361

Peters, M., \& Battista, C. (2008). Applications of mental rotation figures of the Shepard and Metzler type and description of a mental rotation stimulus library. Brain and Cognition, 66(3), 260-264. doi: 10.1016/j.bandc.2007.09.003 
Ploner, M., Lee, M. C., Wiech, K., Bingel, U., \& Tracey, I. (2010). Prestimulus functional connectivity determines pain perception in humans. Proceedings of the National Academy of Sciences, 107(1), 355-360. doi: 10.1073/pnas.0906186106

Rahnev, D. A., Bahdo, L., de Lange, F. P., \& Lau, H. (2012). Prestimulus hemodynamic activity in dorsal attention network is negatively associated with decision confidence in visual perception. Journal of Neurophysiology, 108(5), 15291536. doi: $10.1152 /$ jn. 00184.2012

Rissman, J., Gazzaley, A., \& D'Esposito, M. (2004). Measuring functional connectivity during distinct stages of a cognitive task. Neurolmage, 23(2), 752-763. doi: 10.1016/j.neuroimage.2004.06.035

Sadaghiani, S., Hesselmann, G., \& Kleinschmidt, A. (2009). Distributed and antagonistic contributions of ongoing activity fluctuations to auditory stimulus detection. The Journal of Neuroscience, 29(42), 13410-13417. doi: 10.1523/JNEUROSCI.2592-09.2009

Sadaghiani, S., Poline, J.-B., Kleinschmidt, A., \& D’Esposito, M. (2015). Ongoing dynamics in large-scale functional connectivity predict perception. Proceedings of the National Academy of Sciences, 112(27), 8463-8468. doi: 10.1073/pnas.1420687112

Sali, A.W., Courtney, S.M., \& Yantis, S. (2016). Spontaneous fluctuations in the flexible control of covert attention. The Journal of Cognitive Neuroscience, 36(2), 445-454. doi: 10.1523/JNEUROSCI.2323-15.2016

Sapir, A., D’Avossa, G., McAvoy, M., Shulman, G. L., \& Corbetta, M. (2005). Brain signals for spatial attention predict performance in a motion discrimination task. Proceedings of the National Academy of Sciences, 102(2), 1781017815. doi: 10.1073/pnas.0504678102

Scheibner, H. J., Bogler, C., Gleich, T., Haynes, J.-D., \& Bermpohl, F. (2017). Internal and external attention and the default mode network. Neurolmage, 148, 381-389. doi: 10.1016/j.neuroimage.2017.01.044

Shepard, R. N., \& Metzler, J. (1971). Mental rotation of three-dimensional objects. Science, 171(3972), 701-703. 
Smallwood, J., \& Schooler, J. W. (2015). The science of mind wandering: Empirically navigating the stream of consciousness. Annual Review of Psychology, 66, 487-518. doi: 10.1146/annurev-psych-010814-015331

Soravia, L. M., Witmer, J. S., Schwab, S., Nakataki, M., Dierks, T., Wiest, R., ... Jann, K. (2016). Prestimulus default mode activity influences depth of processing and recognition in an emotional memory task. Human Brain Mapping, 37(3), 924-932. doi: 10.1002/hbm.23076

Tian, L., Jiang, T., Liu, Y., Yu, C., Wang, K., Zhou, Y., ... Li, K. (2007). The relationship within and between the extrinsic and intrinsic systems indicated by resting state correlational patterns of sensory cortices. Neurolmage, 36(3), 68490. doi: 10.1016/j.neuroimage.2007.03.044

Vanhaudenhuyse, A., Demertzi, A., Schabus, M., Noirhomme, Q., Bredart, S., Boly, M., ... Laureys, S. (2011). Two distinct neuronal networks mediate the awareness of environment and of self. Journal of Cognitive Neuroscience, 23(3), 570-578.

Weissman, D. H., Roberts, K. C., Visscher, K. M., \& Woldorff, M. G. (2006). The neural bases of momentary lapses in attention. Nature Neuroscience, $9(7)$, 971-978. doi: 10.1038/nn1727 
PRE-STIMULUS DMN EFFECTS ON COGNITION 



\section{CHAPTER 4}

\section{GRADUAL SELF-REGULATION OF THE DEFAULT MODE NETWORK WITH REAL-TIME FMRI NEUROFEEDBACK}
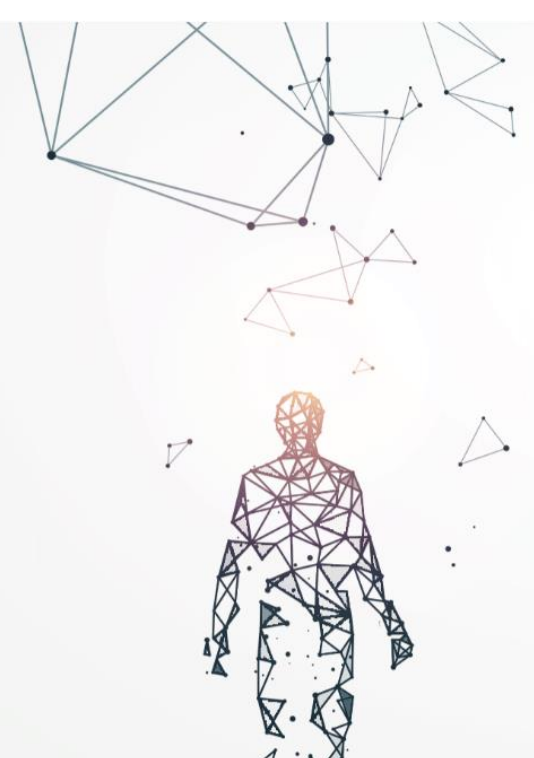

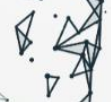

$\therefore$

$\triangle v^{4}$ 


\section{Based on:}

Kamp, T., Sorger, B., Lührs, M., Eck, J., \& Goebel, R. (submitted). Gradual selfregulation of the default mode network with real-time fMRI neurofeedback. 


\begin{abstract}
The default mode network (DMN) has lately been implicated in various cognitive functions and psychiatric disorders and has become a target for diagnostic neuroimaging tools. In fact, modulating the DMN in order to balance alterations or enhance the network's functioning might be a possibility in future therapeutic or cognitive enhancement studies. At the same time, it has been shown that real-time functional magnetic resonance imaging ( $r$-fMRI) neurofeedback provides a powerful tool to gain access to and modulate regional brain activation. To establish whether rt-fMRI neurofeedback promotes gaining targeted control over the DMN, we investigated in this feasibility study whether gradual self-regulation of the DMN can be achieved when providing neurofeedback. Participants $(n=8)$ attended one DMN up-regulation and one DMN down-regulation session in which they used self-episodic memory recall and mental calculation as modulation strategies, respectively, to achieve three different target levels (low, medium and high) while additionally being provided with continuous neurofeedback. The results indicate that neurofeedback did not support a gradual self-regulation of the DMN, compared to self-regulation without neurofeedback. However, the neurofeedback training induced changes in the self-regulation ability without neurofeedback in both sessions: while in the down-regulation session, participants showed a trend for self-regulation improvement after the training, the self-regulation deteriorated after neurofeedback in the up-regulation session.
\end{abstract}




\section{INTRODUCTION}

The so-called default mode network (DMN) is a large-scale network comprised of different hubs which are functionally connected, including the posterior cingulate cortex (PCC)/precuneus, medial prefrontal cortex (MPFC)/ventral anterior cingulate cortex (VACC) and parietal regions (Fox et al., 2005; Fransson, 2005). In the past years, the DMN has been implicated in various cognitive functions (Buckner, Andrews-Hanna, \& Schacter, 2008; Raichle, 2015; Whitfield-Gabrieli \& Ford, 2012), including self-referential processes, such as autobiographical memory, prospection, and Theory of Mind (Buckner et al., 2008; Spreng \& Grady, 2010), and stimulus-independent processing or intrinsic thought, such as daydreaming or mind wandering (Andrews-Hanna, Reidler, Huang, \& Buckner, 2010; Christoff, Gordon, Smallwood, Smith, \& Schooler, 2009; Mason et al., 2007; Scheibner, Bogler, Gleich, Haynes, \& Bermpohl, 2017; Smallwood \& Schooler, 2015). At the same time, alterations within this network have been associated with neurological and neuropsychiatric disorders, as for example Alzheimer's disease, Parkinson's disease, attention deficit hyperactivity disorder, schizophrenia, and mood disorders (Broyd et al., 2009; Mohan et al., 2016). As a consequence, there is a need for developing methods to modulate the DMN state in order to potentially balance or enhance its functioning. A suitable method for this purpose might be real-time functional magnetic resonance imaging (rt-fMRI) neurofeedback, as it allows participants to modulate regional brain activation with the help of real-time feedback from these brain regions (for a review, see Sulzer et al., 2013). While both the investigation of the functions of the $D M N$, as well as applications of rt-fMRI neurofeedback have received increased attention in neuroscientific research in the past years, there is only sparse evidence for the DMN as a target for neurofeedback applications. In one study, it was investigated whether participants were able to down-regulate the PCC as one of the key hubs of the DMN (Zhang et al., 2013). In this study, the researchers compared the regulation ability within the DMN of an experimental group (mental task performance while receiving neurofeedback) to the one of a control group (mental rehearsal without neurofeedback). While the control group performed imagery of a finger tapping sequence throughout the regulation periods in the two modulation runs, the experimental group additionally received real-time information about their PCC activation level. It was shown that the experimental group showed a lower PCC activation compared to the control 
group in the second training run, however, the PCC activation in the control group was increased in the second modulation run compared to the first. Another rt-fMRI neurofeedback study compared the neurofeedback success in one key hub of the DMN, namely the inferior parietal lobe (IPL), and in five visual areas, including early and higher-order visual cortex (Harmelech, Friedman, \& Malach, 2015). The results indicate that participants vary in their ability to modulate these regions with the neurofeedback training, with the highest regulation taking place for the DMN neurofeedback target region, with decreasing regulation success when going down the visual hierarchy. This suggests that the DMN could potentially be a network which can be gradually self-regulated with neurofeedback.

Another set of rt-fMRI (neurofeedback) studies investigated the link between one key hub of the DMN, the PCC, and meditation experience. In one experiment, experienced meditators and meditation novices were provided with real-time information about their PCC activation while meditating (Garrison, Scheinost, et al., 2013). Throughout the meditation, they were probed to report their subjective experience of the meditation at given time points and compare this experience to the PCC activation at that time. In both meditators and novices, the correspondence between PCC activation and subjective meditation experience was high. However, when asked to self-regulate the PCC activation with the help of the neurofeedback information, experienced meditators were more able to volitionally down-regulate their PCC activation than novices. In another experiment by the same research group, participants reported their cognitive experience corresponding to the PCC activation after each real-time neurofeedback run (Garrison, Santoyo, et al., 2013). The results indicated that an increased PCC activation was associated with the subjective experience 'distracted awareness', while a decreased PCC activation was related to 'effortless awareness'. This research suggests that a volitional control of the DMN might be a desirable goal to increase active, but effortless awareness. However, the results also suggest that without any meditation experience, self-regulation of the DMN might be difficult. While the abovementioned studies all provide interesting insights in the possibility to self-regulate the DMN with neurofeedback, they all used only one key hub (the $\mathrm{PCC}$ or IPL) of the DMN as the neurofeedback target region. However, as mentioned above, the DMN encompasses various brain regions, with main hubs in PCC/precuneus, MPFC/vACC, and parietal regions (Fox et al., 2005; Fransson, 2005). 
Thus, how far the interplay of these regions can be modulated using rt-fMRI neurofeedback still remains unknown. Moreover, it still remains unclear how well the DMN can be voluntarily modulated both in a positive direction (up-regulation) and in a negative direction (down-regulation). Inspired by earlier exploratory studies (Goebel, Sorger, Kaiser, Birbaumer, \& Weiskopf, 2004), neurofeedback paradigms have been recently introduced which aim at gradually self-regulating brain areas to specific target levels (Krause et al., 2017; Sorger et al., 2018). This paradigm allows to investigate the control over a brain region in a more sensitive way, as participants are not merely asked to maximise or minimise their brain activation magnitude with the help of the neurofeedback information, but rather use the information to gradually achieve certain levels of activation. This provides a measure of how well a brain region can be controlled precisely, as the activation needs to be controlled with respect to target levels, rather than maximizing or minimizing the activation without specific targets. This might be advantageous, as participants have more information about the success of their self-regulation (vs. potential overshooting in response to a maximizing or minimizing approach) and therefore might learn from the feedback in a faster way. Moreover, this gradual self-regulation paradigm might be beneficial in future neurofeedback applications which aim at modifying behaviour, as different performance levels can be induced by different activation levels.

Based on this background, we here conducted an rt-fMRI neurofeedback feasibility experiment, to assess whether participants are able to both up- and down-regulate the DMN to different target levels. Furthermore, we hypothesised that participants would be more efficient to self-regulate the DMN when provided with neurofeedback information compared to self-regulation without neurofeedback. Moreover, we hypothesised that participants would be able to learn from the neurofeedback training, resulting in a better self-regulation performance after the training than before the training.

\section{MATERIALS AND METHODS}

\section{Participants}

Eight healthy volunteers (mean age: $26.9 \pm 2.1$ SD years, four female, two lefthanded), with normal or corrected-to-normal vision participated in the study. 
Participants gave written informed consent before the experimental sessions and were monetarily compensated for their participation. The experimental procedure was approved by the Ethics Review Committee Psychology and Neuroscience at Maastricht University.

\section{Experimental procedure}

\section{General procedure}

Each participant attended one initial training session and two rt-fMRI neurofeedback scanning sessions, one of which aimed at up-regulating and the other at down-regulating the DMN to different target levels. Participants were instructed to perform and to modulate two different mental tasks (one for each regulation direction) in order to reach different target levels. Similar as in Sorger et al. (2018), participants were asked to modulate their brain activation to three different target levels (low, medium, and high).

\section{Mental strategies and training session}

In the training session, participants were informed about the general procedure of the two fMRI sessions, neurofeedback as a tool, the hemodynamic delay and noise present in the data. Furthermore, they were trained on the two mental tasks which were used as strategies to up- and down-regulate the DMN in the MRI neurofeedback sessions. We employed self-episodic memory recall and mental calculation as regulation strategies in the up-regulation and down-regulation session, respectively. These two mental tasks have been shown to activate the same neuronal populations in the PCC using intracranial electroencephalography (Foster, Dastjerdi, \& Parvizi, 2012). While self-episodic memory activates these neuronal populations, their response was suppressed during arithmetic calculations, resulting in a deactivation of their response.

In the self-episodic memory task to up-regulate the DMN, participants were instructed to recall an autobiographical memory. For this, we asked participants to select a positive life event which they could re-live repeatedly, as vivid as possible and with great detail. We further asked to think of two back-up memories in case the selected memory would not result in sufficient brain activation throughout the up-regulation session. We further suggested modulation strategies for the mental 
tasks to the participants in order to regulate their brain activation to different levels. These strategies included adding more sensory details (e.g. smell, colours of the particular scene) and feelings to the recalled memory, or remembering the interaction with people taking part in this life event.

For the down-regulation session, we instructed participants to perform mental calculation by calculating simple sums, starting with the number 5 and adding a particular number continuously until reaching 100 . For each participant, we selected an individual number to use for adding in which they could perform the mental calculation at a constant speed while still calculating correctly. We instructed participants to vary mental calculation performance according to the target level in the MRI session by using more difficult numbers for adding or increasing the speed of calculating. Both tasks were also checked in the training session by mocking the timing in the MRI scanner and asking participants to verbally perform the tasks.

\section{FMRI neurofeedback sessions}

Each participant underwent one up- and one down-regulation session of the DMN in the MRI scanner, partially accompanied with rt-fMRI neurofeedback. The order of the up- and down-regulation session was balanced across participants. Each scanning session consisted of one anatomical run, one resting state run, one functional localiser run, one modulation run without neurofeedback (NoNF), three neurofeedback runs (NF), followed by one last NoNF run, resulting in a total scanning time of $1 \mathrm{~h} 12 \mathrm{~min}$ (Fig. $1 \mathrm{~A}$ ). The resting state run and the functional localiser run were used to define an individual DMN neurofeedback target region (see section 'Online definition of the neurofeedback target $\mathrm{DMN}^{\prime}$ '). In the resting state run (11 min and 15s), participants were instructed to keep their eyes open, focus on the fixation cross in the middle of the screen and to not think of anything in particular. The subsequent functional localiser run (7min and 10s) consisted of an initial 30s rest period, followed by five mental calculation and five self-episodic memory task blocks of 20s (visually presented with the words 'Recall memory' and 'Mental calculation'), alternated with 20s rest periods indicated by a fixation cross (Fig. 1B). Participants were asked to maximise their regulation for the given mental task in each block. 
During the following NF and NoNF runs (Fig. 1C), participants were instructed to self-regulate their brain activation to three different target levels (low, medium and high) equivalent to Sorger et al. (2018). For this, participants were presented with a thermometer-like display, consisting of ten vertically stapled rectangles on a black background. The target level to which participants were asked to regulate their brain activation to was indicated by a red outline of the respective rectangle. The low, medium and the high level were represented by rectangle 3, 6 and 9 on the thermometer graph, respectively. Both NoNF and NF runs started with one initial rest period (30s) and one test ("warm up") trial (either the low, medium, high level, presented randomly across runs), which was further disregarded in the analyses. After the test trial, the runs consisted of twelve task blocks, with four blocks of each target level presented in a random order. Each target level block was presented for $20 \mathrm{~s}$, alternated by $20 \mathrm{~s}$ rest periods, resulting in a run length of $9 \mathrm{~min}$ and $10 \mathrm{~s}$. For the NoNF runs, participants were instructed to intuitively modulate their individual mental task strategy to achieve the different brain activation levels according to the instructed target level by changing specific aspects of mental task performance (see description of the mental tasks in the results section). Participants received the same instructions for the NF runs, however, they were additionally asked to systematically modulate their mental task performance based on the presented neurofeedback information that they continuously received from their blood oxygenation level dependent (BOLD) response in their neurofeedback target DMN. This feedback information was visualised by filled rectangles on the thermometer display. Importantly, participants were not aware of the target region for the neurofeedback, nor that the two session included different directions of regulation (up- and down-regulation). They were rather instructed to use the presented feedback in order to self-regulate their brain activation level by changing aspects of the mental strategy to be used in that particular session. In line with this, they were presented with an inverted feedback signal for the down-regulation session, meaning that they only saw neurofeedback information in the positive direction on the screen, although the DMN activation was expected to be mainly negative.

After each session, participants received a questionnaire documenting the content of their mental task and the modulation strategies with which they varied the task for each level. 
A

SESSION:

Up-regulation

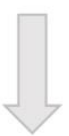

Modulation Strategy:

Self-episodic memory
$X_{n=8}^{R}$

Runs:

Anatomy

Resting state

Functional localizer

B

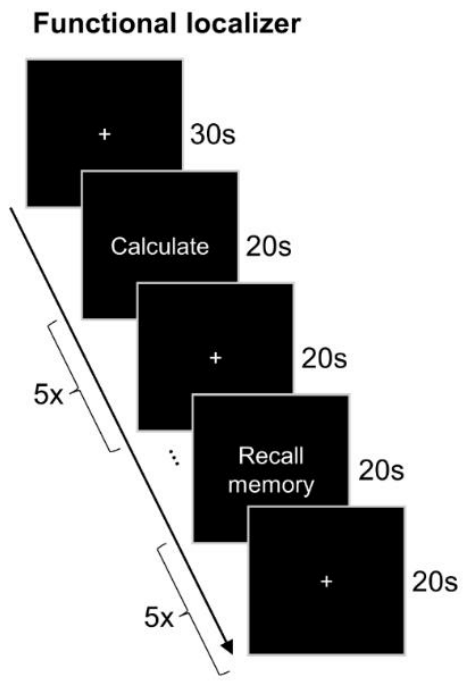

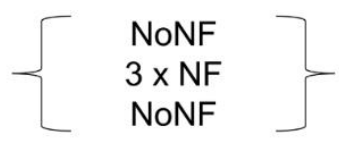

Modulation Strategy:
Mental calculation
SESSION:

Down-regulation

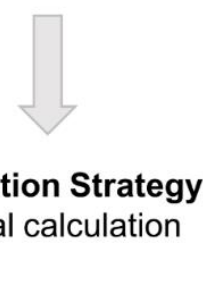

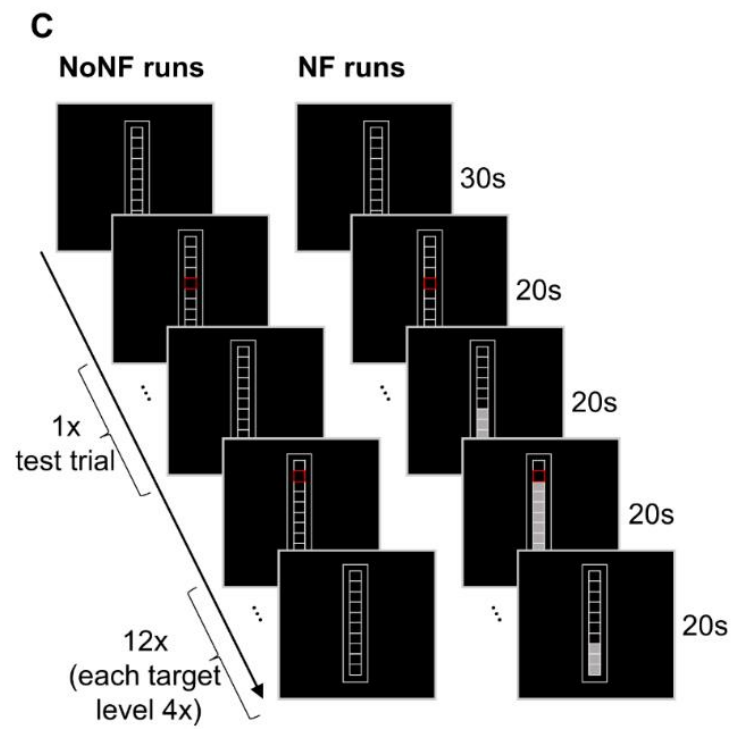

Figure 1: Experimental procedure. A. Overview of the order of runs in the DMN up- and down-regulation session. B. Time-resolved overview of the functional localiser run. After an initial baseline (30s), participants performed five mental calculation blocks and five self-episodic memory recall blocks (each 20s), interleaved by rest periods (20s). C. Time-resolved overview of the NoNF and the NF runs. Both run types started with an initial baseline (30s) and one test regulation block (20s), in which participants did not receive neurofeedback information, disregarding the run type. After this, participants regulated their DMN activation according to the displayed target level (indicated by a red rectangle) or rested when the outline of all rectangles remained white. In total, there were twelve trials in each run with four blocks of each target level, presented in random order. In the NF runs, participants additionally received information about their volume-to-volume activation within the $\mathrm{DMN}$ and were asked to modulate their regional brain activation accordingly.

Abbreviations: NoNF, runs without neurofeedback; NF, neurofeedback runs; s, seconds. 


\section{Data acquisition}

Data acquisition for both anatomical and functional MRI data was performed using a 3T whole-body MAGNETOM PrismaFit scanner (Siemens AG, Erlangen, Germany). In each scanning session, participants were provided with ear protection and foam padding to reduce head motion when being comfortably placed in the scanner. In each scanning session, T1-weighted anatomical scans were obtained for every participant using a three-dimensional (3D) magnetization-prepared rapidacquisition gradient-echo (MPRAGE) sequence (192 slices, slice thickness $=1 \mathrm{~mm}$, no gap, $\mathrm{TR}=2250 \mathrm{~ms}, \mathrm{TE}=2.21 \mathrm{~ms}, \mathrm{FA}=9^{\circ}$, FOV $=256 \times 256 \mathrm{~mm}^{2}$, matrix size $=256 \times 256$, total scan time $=8 \mathrm{~min} 26 \mathrm{~s})$. All functional images were acquired using multiband (MB) accelerated echo-planar imaging (EPI) (48 slices, voxel dimensions $=3 \mathrm{~mm}$ isotropic, no gap, $\mathrm{TR}=1000 \mathrm{~ms}, \mathrm{TE}=31 \mathrm{~ms}, \mathrm{FA}=62^{\circ}$, FOV $=228 \times 228 \mathrm{~mm}^{2}$, matrix size $=76 \times 76, \mathrm{MB}$ factor $=4$, slice order $=$ interleaved) (Moeller et al., 2010). Functional data acquisition consisted of one resting state run (675 volumes), one functional localiser run (430 volumes), one NoNF run (550 volumes), three NF runs (550 volumes each) and one final NoNF run (550 volumes).

\section{Online data analysis}

Online data analysis was performed using Turbo-BrainVoyager 4.0.B1 (Brainlnnovation B.V., Maastricht, The Netherlands), BrainVoyager 20.6 (Brainlnnovation B.V., Maastricht, The Netherlands) and MATLAB 2014a (The MathWorks, Inc., Natick, Massachusetts, United States).

\section{Online pre-processing of imaging data}

After the anatomical data were fully acquired, they were corrected for intensity inhomogeneity and transformed into Talairach stereotactic space. All functional runs were aligned to the first volume of the resting state run and corrected for motion in real-time, using three rotations and three translations. Just after the acquisition of the resting state run, a temporal high-pass filter (cut-off value of $0.008 \mathrm{~Hz}$ ) was additionally applied on the time series to prepare the resting state data analysis. The run was moreover co-registered to the anatomical scan and transformed into Talairach space. The remaining functional runs (localiser, NoNF, NF runs) were spatially smoothed using a Full Width at Half Maximum of $4 \mathrm{~mm}$. 


\section{Online definition of the neurofeed back target DMN}

The individual definition of the neurofeedback target DMN was accomplished online in two major consecutive steps. First, two seed-based analyses were performed based on the data of the resting state run, one with a seed in the $\mathrm{PCC} /$ precuneus and one with a seed in the MPFC/vACC. For each of these analyses, the averaged time course of a $10 \mathrm{~mm}$ sphere surrounding the centre of the seed region (Talairach coordinates for $P C C /$ precuneus: $x=-4, y=-46, z=37$; for MPFC/vACC: $x=-1, y=42, z=-4$ ) was extracted from the resting state time series. The respective coordinates were selected based on a meta-analyses about these hubs analysed from resting state data (Fox et al., 2005). The seed based analyses were done by performing a general linear model (GLM), including the time course of the respective seed region as a predictor, alongside six motion parameters (three rotations and three translations), the signal from the ventricles, as well as the signal from the white matter (both time series extracted from the segmented ventricles and white matter, respectively) as confounding predictors (all z-transformed). The resulting activation maps were thresholded $(p=0.001)$ and the overlap between the two maps was calculated. As we were interested in regulating the main hubs of the $D M N$, we selected all active voxels in the overlap map surrounding the $\mathrm{PCC} /$ precuneus and MPFC/VACC defined those as the individual DMN.

As a second step, the individual DMN was analysed with respect to the activation from the functional localiser run. As soon as the data of the localiser run were acquired, a GLM was calculated with predictors for the mental calculation and self-episodic memory task blocks. A contrast was specified which investigated which voxels showed a higher activation for self-episodic memory than for mental calculation and were significantly larger than 0 . The $30 \%$ "best" voxels within the DMN definition based on the resting state were selected and defined as the neurofeedback target $\mathrm{DMN}$, hereby ensuring that voxels in the two main hubs of the DMN (the PCC/precuneus and the MPFC/vACC) were included.

\section{Neurofeedback Calculation}

The NF runs were analysed in real-time as soon as their volumes became available. Each volume of a run was initially aligned to the first volume of the resting state data to correct for potential head movements throughout the experiment. For each time point, the time course signal was averaged across all voxels within the 
individual neurofeedback target DMN. To generate the neurofeedback information, a baseline was determined based on the rest period before the first modulation block and updated for each subsequent modulation block (sliding baseline). To account for the sluggishness of the BOLD response, only the activation from the last 14 time points (14s) of each rest period, as well as two time points (2s) into the modulation block were used to calculate the average baseline for each block. The percent signal change (feedback value) for each time point was then calculated based on the following equation:

$$
\text { Feedback value }=\left(\frac{\text { signal value }- \text { baseline }}{\text { baseline }}\right) * 100
$$

The resulting feedback value was consecutively related to the maximum percent signal change (Max-PSC with a minimum of $1 \%$ ) as specified in the functional localiser for the task blocks of the current regulation session ( $\left(\frac{\text { Feedback value }}{\text { MaxPSC }}\right)$; for up-regulation: Max-PSC of self-episodic memory condition; for down-regulation: Max-PSC of mental calculation condition). Subsequently, the values were clipped in the range of [0.0-1.0] and multiplied by 10 to get the neurofeedback display value to fill the appropriate rectangles on the thermometer. Note that for the down-regulation session, feedback values were additionally inverted so that participants would only see feedback in the positive direction. Neurofeedback information was updated continuously for each time point (each volume) and displayed to the participant immediately after becoming available.

\section{Offline data analysis}

\section{Pre-processing of imaging data}

Anatomical data were corrected for spatial intensity inhomogeneity, after which they were normalised into Talairach stereotactic space. Functional data were pre-processed using 3D motion correction, slice scan time correction, linear trend removal and a temporal high-pass filtering (cut-off value: $0.008 \mathrm{~Hz}$ ). The individual neurofeedback target DMNs were transformed into 3D regions of interest and transformed into Talairach stereotactic space. 


\section{Mean self-regulation of the DMN and primary task areas in NoNF and NF runs}

For both up- and down-regulation sessions separately, we carried out a fixed effects group GLM analysis to investigate the activation for the different target levels within the neurofeedback target DMN in the NoNF and NF runs. The GLM included predictors for the target levels (low, medium, and high), and for the information provided to the participants (NoNF and NF), as well as six motion parameters (three rotations and three translations) as confounding predictors. Based on the resulting beta values, we conducted a second-level two-way repeated measures analysis of variance (ANOVA, F-test) to test whether the interaction between the target level and the nature of the feedback information provided to the participant was significant. Furthermore, we performed a contrast analysis, testing whether NF runs evoked larger responses within the DMN than the NoNF runs. On a descriptive level, we looked at the activation in individual runs to investigate how self-regulation of the DMN would manifest with increasing training time. For this, we carried out single-run GLMs with predictors for each target level.

Next to analysing the activation within the neurofeedback target DMNs, we additionally checked how primary task regions for both mental tasks responded to the modulation blocks. We hereby specified the hippocampus as the primary task region for self-episodic memory recall (up-regulation session) and the intraparietal sulcus (IPS) as the primary task region for mental calculation (down-regulation session). Even though these areas were not the target regions for the neurofeedback, we still assumed a gradual modulation of these primary task regions in response to the modulation of the given mental task. This served as a check whether participants actually modulated the task according to our instructions. In order to define these primary task regions, a whole-brain GLM was calculated across the two functional localiser runs for all participants, with predictors for mental calculation and self-episodic memory task blocks. The resulting activation maps for mental calculation and self-episodic memory were consecutively thresholded (cluster-size thresholded with an initial threshold at $\mathrm{p}=0.001$ ) and probability maps were created for each task separately. Subsequently, we defined the cluster surrounding the hippocampus as the primary task area for self-episodic memory recall and the cluster surrounding the intraparietal sulcus as the primary task area for mental calculation. For each 
participant, we then created individual primary task regions by masking the individual activation with the probability maps.

\section{Single-trial absolute distance of DMN activation to target level activation}

To statistically investigate how well participants could modulate their brain activation to the different target levels, we additionally calculated the absolute distance of their neurofeedback target DMN activation to the instructed target level. For each session separately, we calculated the average absolute difference between the indicated target level and the activation within the DMN in each single trial modulation block (see Fig. 2 for a schematic representation of the analysis). For this, we extracted the feedback values for all time points of each trial (to account for the hemodynamic delay, feedback values of 20 time points (thus 20s) were extracted with a shift of +2 after trial onset) and calculated the corresponding feedback display value (calculated according to the equation in the neurofeedback calculation section above, but as there was no need for displaying the values, we refrained from clipping to allow for all values to be integrated in the analysis). Then, the absolute difference between the target level in the given modulation block and the feedback display value was calculated for all time points of the trial. The resulting values were averaged such that each modulation block trial had one absolute mean distance value to the target level. Subsequently, three tests were performed investigating different hypothesis with different run combinations. First, we investigated whether the absolute distance between the target level and the actual achieved activation was different between NF and NoNF runs. For this, we averaged across all trials in the NF and NoNF conditions respectively and performed a one-sided paired-samples t-test, as we hypothesised that the absolute distance should be lower in the NF condition than in the NoNF condition. Second, we were interested whether participants improved in using feedback information to regulate their DMN activation over time. Therefore, we averaged the absolute distance values across all trials for each feedback run separately and calculated a one-way ANOVA to test whether there was a difference in the absolute distance to the target level between the three NF runs (F-test). Third, we compared the absolute distance to the target level of the first NoNF run with the last NoNF run to see whether participants would have learned from the performed neurofeedback runs to regulate the $\mathrm{DMN}$, even when no neurofeedback information was 
displayed. For this, we calculated a one-sided, paired-samples t-test to test the difference between the absolute distance of the brain activation to the target level in the NoNF run at the beginning of the session and the NoNF run at the end of each session. 


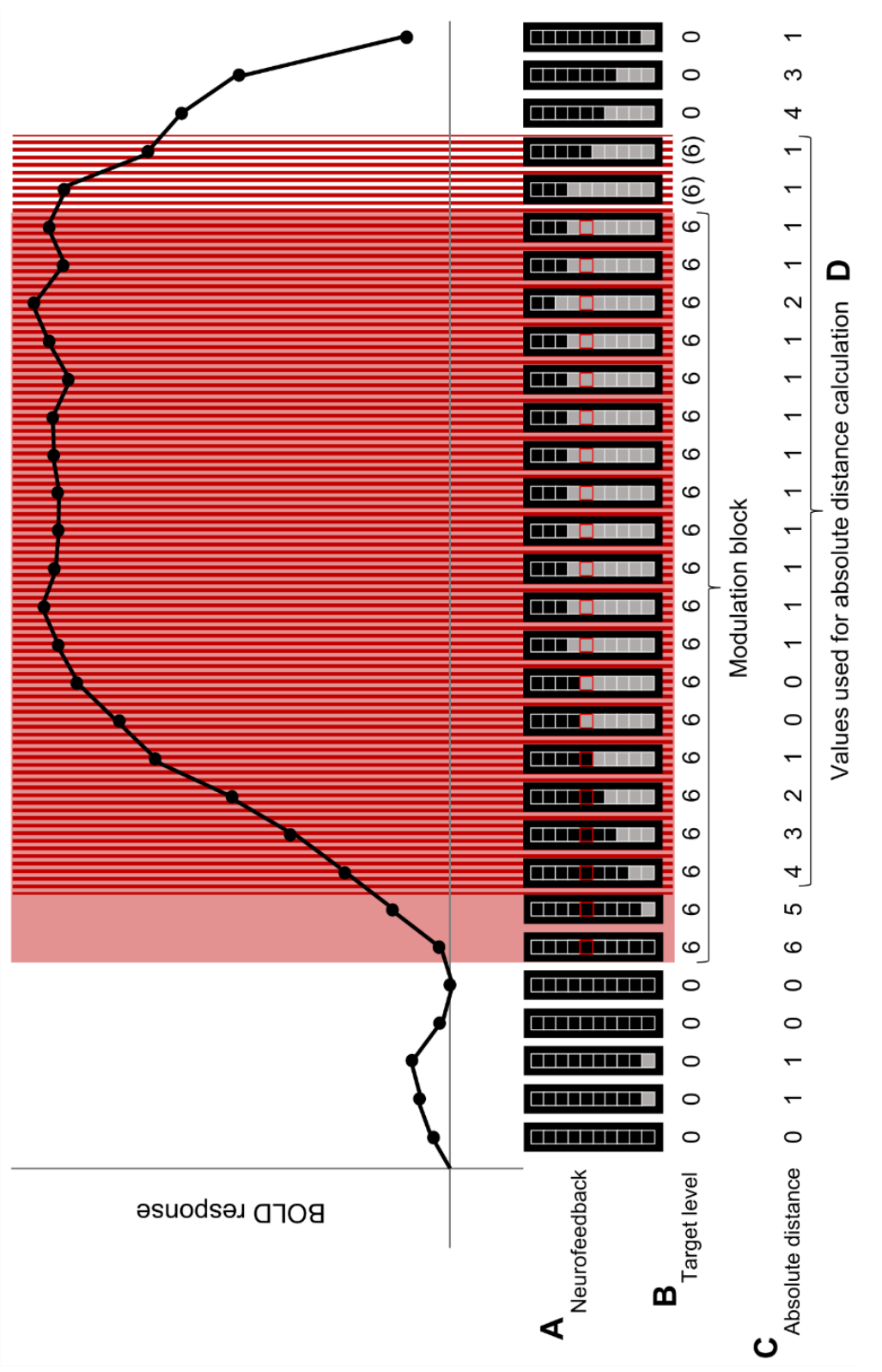

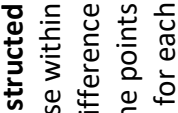

站

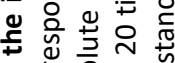

응

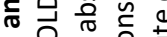

듣 은 号

的这

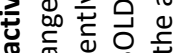

乙

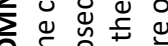

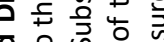

용

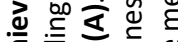

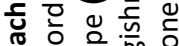

๑ U 은

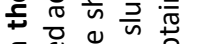

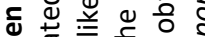

$\frac{\pi}{5} \frac{1}{5}+\frac{5}{4}$

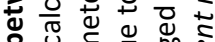

ㅇ

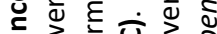

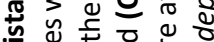

万人

ॠ

흥 흥 흐음

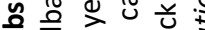

둥 $\frac{\pi}{2}=\frac{n}{0}$

둥ㅎㅎ응

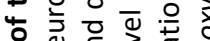

ᄃ $\frac{1}{\pi} \frac{\pi}{5}$

을 뜬 눙용

त

उัँ

능 흔

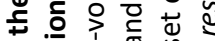

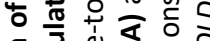

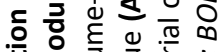

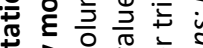

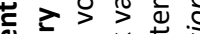

元的兑苋吉

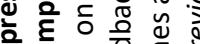

ब ब

.

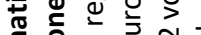

$\varepsilon$ 눠

든 잉

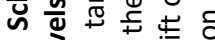

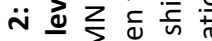

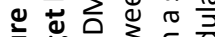

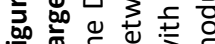

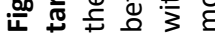




\section{RESULTS}

\section{Online definition of the neurofeedback target DMN}

For each session of each participant, a neurofeedback target DMN could be identified, based on which the neurofeedback information was generated during the NF runs (Fig.3). Due to technical difficulties in the second session of participant P06, we needed to define the neurofeedback target DMN of this session from the resting state DMN definition of the first session. This was achieved by using the DMN definition from the resting state session (in 3D Talairach stereotactic space) and back-transformed it to the native anatomical data of the current session. Subsequently, we defined the neurofeedback target DMN (as for the remaining participants) using the functional localiser in addition to this DMN to identify the $30 \%$ best voxels responding with a higher activation for self-episodic memory than for mental calculation and were significantly larger than 0 . 

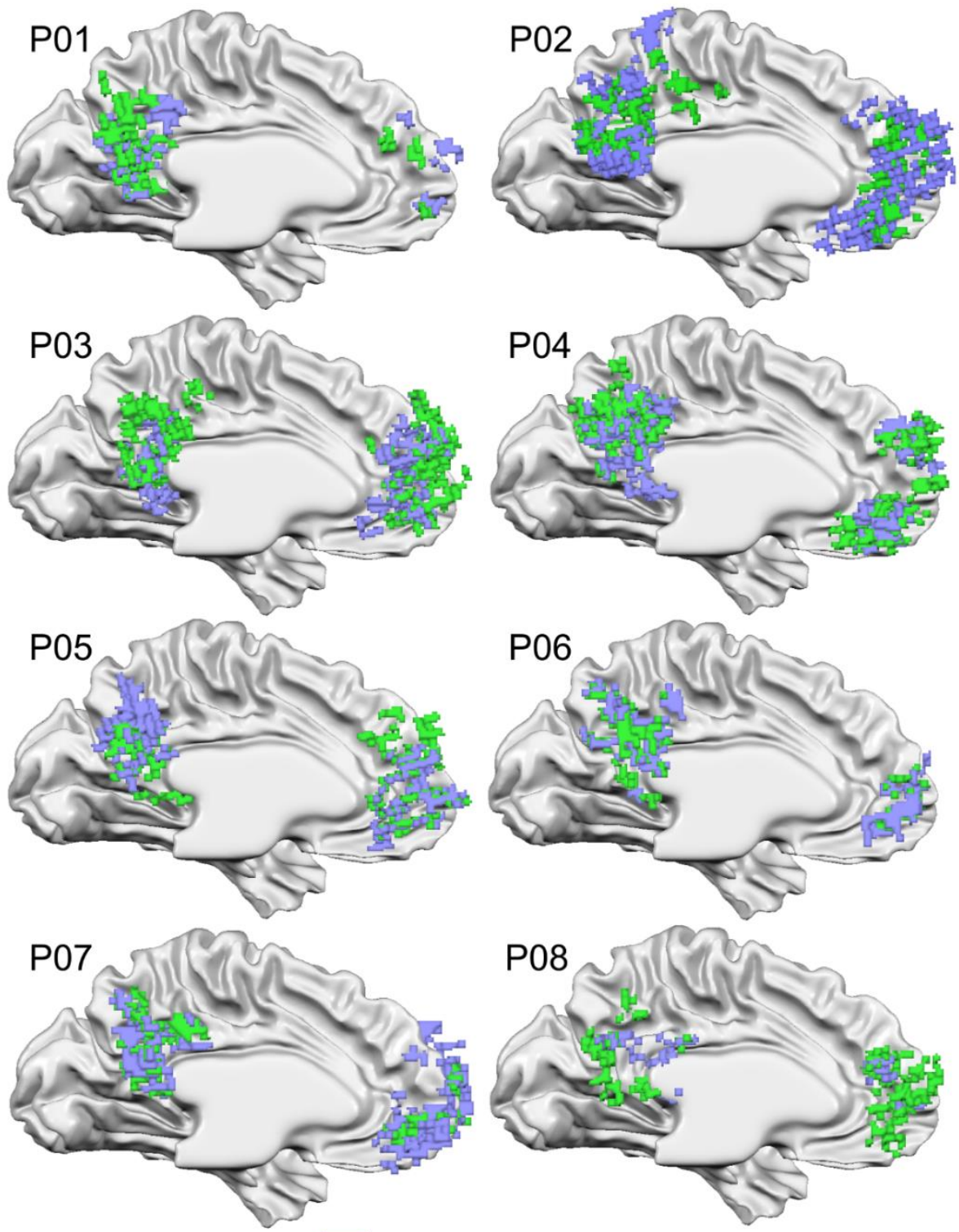

Up-regulation session

Down-regulation session

Figure 3: Individual neurofeedback target DMNs. The green voxels indicate the neurofeedback DMN target region for the up-regulation session, while the blue voxels represent the neurofeedback target DMN for the down-regulation session in each individual. 


\section{Individual self-regulation strategies}

Up-regulation session: Participants generally chose happy life events as their mental strategy for self-episodic memories, as festive occasions, events with significant others, or emotional events. In order to self-regulate their activation to the different target levels, participants mostly changed aspects of the memory or recalled different memories. In general, for the low level, participants reported to have recalled only the key elements or events of the memory, while they increased the level of detail in the memory for the medium level, and finally remembered their emotions for the highest level.

Down-regulation session: Participants varied their mental calculation performance for the three different levels mostly by calculating slow and with low numbers for the low level, while they varied their calculation with higher numbers and/or higher speed for the medium and high levels.

\section{Mean self-regulation levels within the DMN during NoNF and NF runs}

Up-regulation session (Fig.5A): When looking at the mean activation level within the individual neurofeedback target DMNs, the interaction between the target levels (low, medium, high) and the information of feedback (NoNF, NF) was not significant (Fig.5A, upper panel, $F[2,14]=0.112, p=0.895$ ). However, the contrast analysis to test whether the activation was different in the NoNF compared to the NF runs was significant, with a higher activation throughout NoNF compared to NF runs (Fig.5A upper panel, $t=-3.33, p<0.001$ ). Splitting the NoNF and NF activation in separate runs, we investigated how the modulation of the DMN developed over time (Fig.5A, middle panel). When descriptively looking at individual runs across participants, we observed an initial modulation success in the first NoNF run, characterised by a gradual modulation of the DMN across target levels. Surprisingly, this was followed by a reversal of this success in the first NF run, where the activation of the DMN drops below zero. Throughout the second and third NF run, the activation pattern across the three target level conditions seems to improve. However, in the last NoNF run, the performance drops again for all three target levels. When inspecting DMN activation data from individual subjects, similar effects can be found in the majority of participants (Supplementary Material Fig.S1). At the same time, the response in the primary task region for this session 
(hippocampus, Fig.4A) shows a different pattern (Fig.5A, lower panel). While there is a drop in the activation across all target levels from the first NoNF to the first NF run, the amplitude of the responses stay similar across the NF runs, while the gradual modulation across the target levels seem to improve. Furthermore, there is an activation drop in the last NoNF run.

Down-regulation session (Fig.5B): The interaction between target levels (high, medium, low) and the information of feedback (NoNF, NF) was not significant (Fig.5B, upper panel, $F[2,14]=2.728, p=0.1$ ). Contrast analysis to test whether the activation within NoNF runs was different from the activation within NF runs was significant with a higher deactivation of the DMN for the NoNF than the NF condition (Fig.5B, upper panel, $t=-5.87, p<0.001$ ). When looking at individual runs (Fig.5B, middle panel), the activation associated with the target levels varies across runs without a clear pattern. The activation in the primary task region of the IPS (Fig.4B) shows a consistent activation pattern with the three target levels with a clear gradual increase in activation for higher target levels across all runs (Fig.5B, lower panel). Furthermore, no specific pattern could be observed from the DMN activation data of the individual participants (Supplementary Material Fig.S2). 


\section{A Hippocampus}

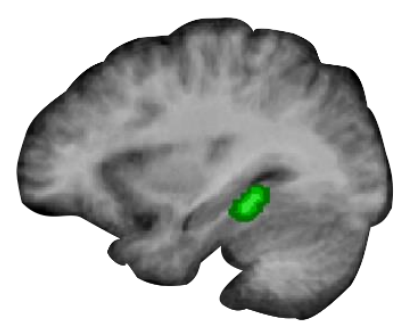

B Intraparietal sulcus

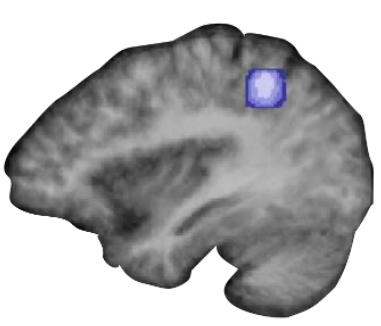

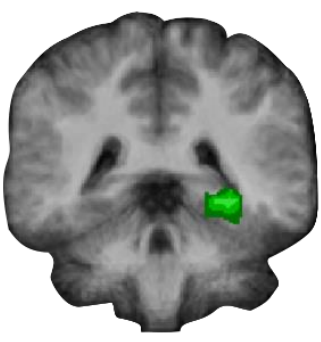
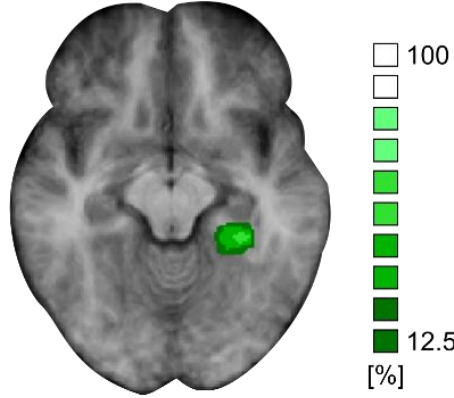

[\%]
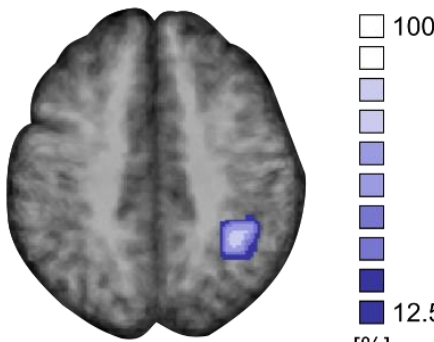

Figure 4: Probability maps of the primary task regions in the up-regulation and down-regulation session. A. The probability map of the individual hippocampus definitions based on the self-episodic memory recall task in the functional localiser. B. The probability map of the individual IPS definitions based on the mental calculation task in the functional localiser.

Remarks: The colour bar indicates how many percent of participants overlap in their definition of the primary task area (with $12.5 \%$ displaying one participant). 
A
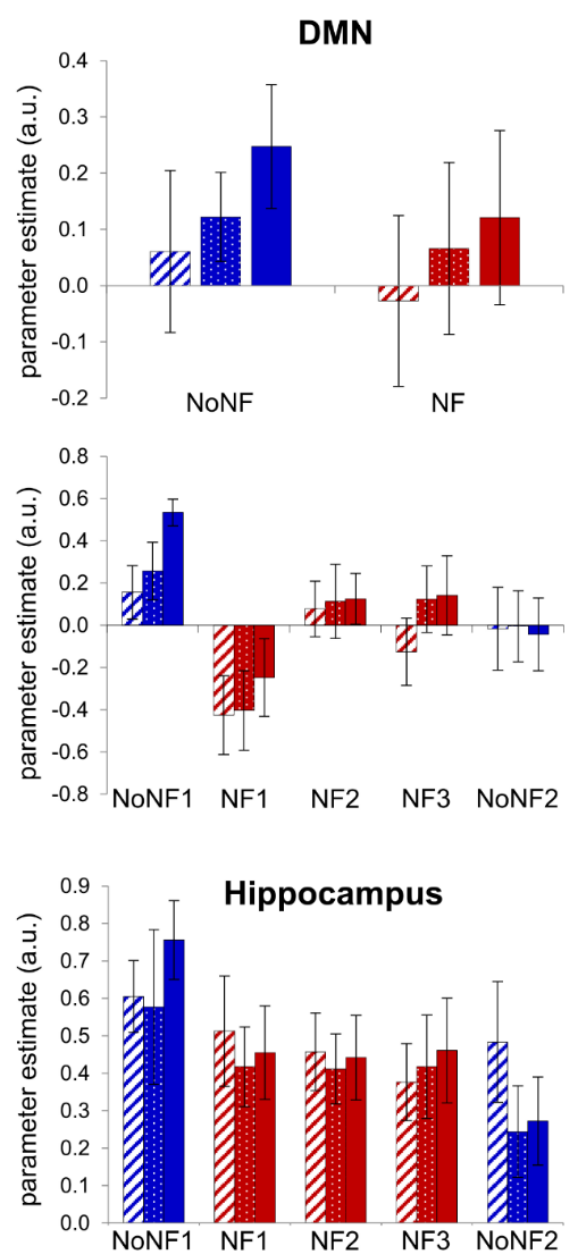

B Down-regulation
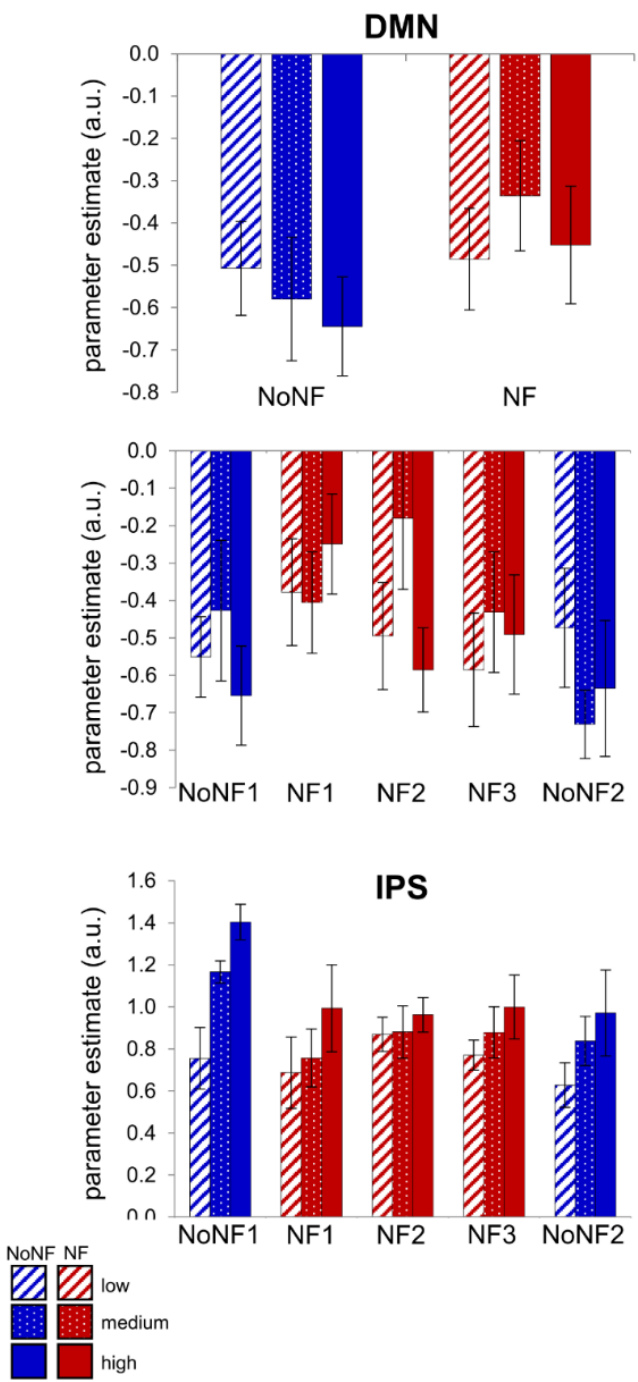

Figure 5: Mean group activation levels within the neurofeedback target DMNs and the primary task regions for both up- and down-regulation sessions. A. Up-regulation session. B. Down-regulation session. Upper panels: The group average activation levels (beta values) within the neurofeedback target DMN across the three different target levels conditions (stripes: low level; dots: medium level; filled: high level) is shown averaged across the NoNF (blue) and the NF runs (red). Middle panels: The group average activation within the neurofeedback target $D M N$ in response to the three target levels for each run separately. Lower panels: The group average activation within the primary task regions (hippocampus for self-episodic memory task [up-regulation session]; IPS for the mental $\rightarrow$ 
calculation task [down-regulation session]) across the three different target level conditions for each run separately.

Remarks: Error bars indicate the standard error of the mean.

Abbreviations: DMN, Default Mode Network; a.u., arbitrary unit; NoNF, no neurofeedback run; NF, neurofeedback run; IPS, intra-parietal sulcus.

\section{Assessing the gradual self-regulation ability by comparing the absolute distance of DMN activation to the target level}

Up-regulation session: The absolute distance between the target level and the actual achieved activation was not significantly different between NF and NoNF (Fig. 6A, upper panel, t[7] $=0 .-0.134, p=0.44$, one-sided). Moreover, the three NF runs did not differ in their distance of the activation to target level (Fig. 6A, middle panel, $F[2,14]=0.337, p=0.719$ ). Furthermore, when comparing the first to the last NoNF run, there was no difference in the distance of the activation within the DMN to the target levels when testing one-sided. We hypothesised that participants should have benefitted from the neurofeedback training and therefore should have shown a lower absolute distance to the target levels in the last NoNF run compared to the first NoNF run. However, if this difference had been tested two-sided, the test would have been significant, showing a higher distance in the activation to the target level (Fig. 6A, lower panel, $t[7]=-2.955, p=0.021$ ).

Down-regulation: The distance between the activation within the neurofeedback target DMN and the target level was not different across NoNF and NF runs (Fig. 6B, upper panel, $t[7]=1.479, p=0.09$, one-sided). There was also no difference in the distance between the DMN activation and the target level across the three NF runs (Fig. 6B, middle panel, $F[2,14]=0.035, p=0.97$ ). However, there was a decrease in the distance between the DMN activation and the target level from the first NoNF run to the last NoNF run, which just missed significance (Fig. 6, lower panel, $\mathrm{t}[7]=1.855, \mathrm{p}=0.05$, one-sided). 
A
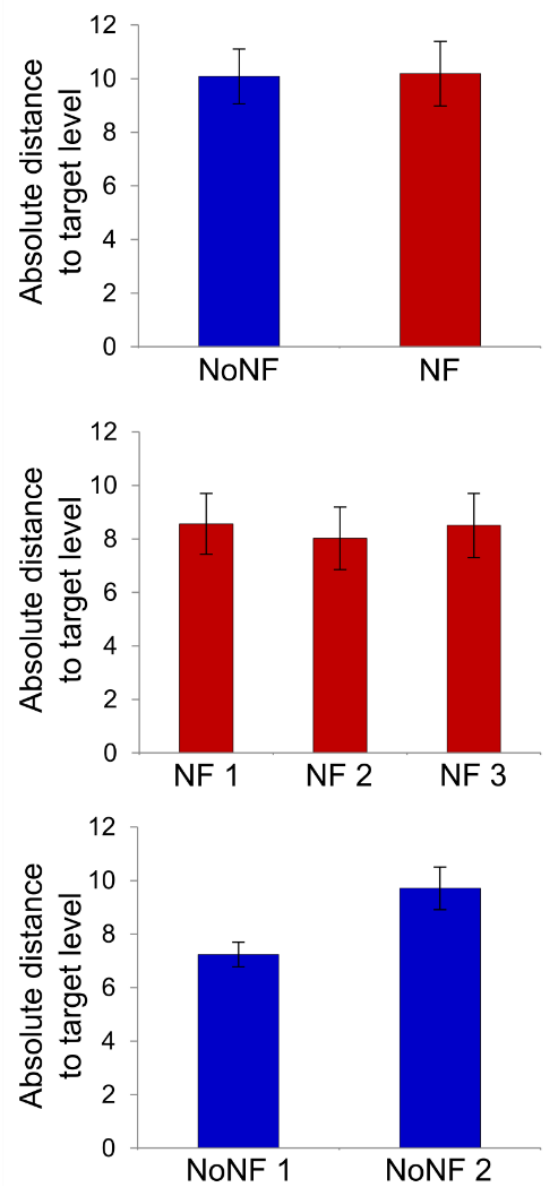

B Down-regulation
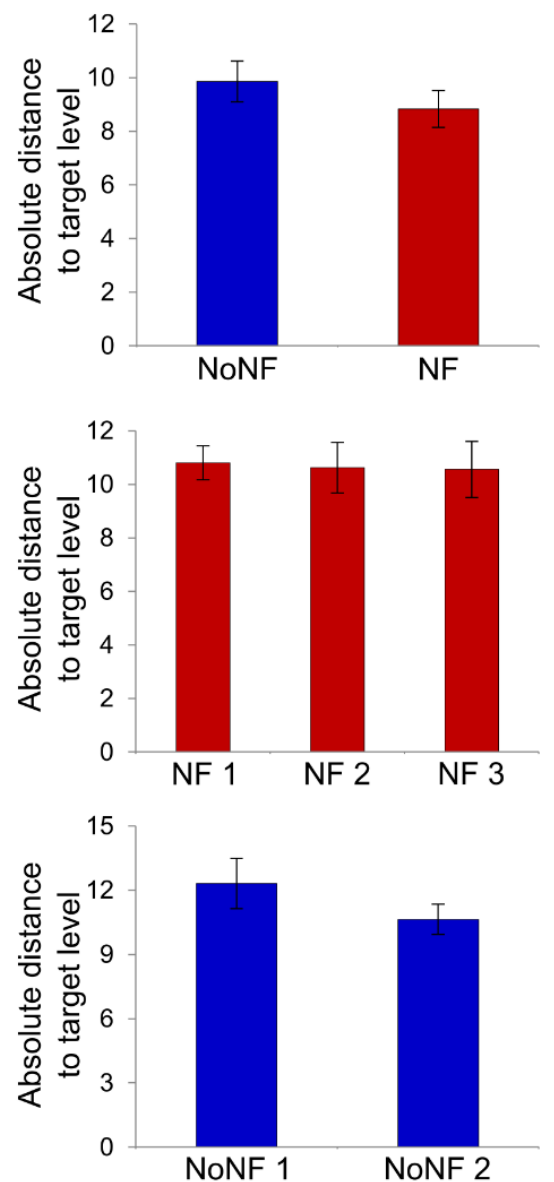

Figure 6. The absolute distance of the activation within the DMN to the target levels in the up-and down-regulation session. A. Up-regulation session. B. Down-regulation session. Upper panels: The group average absolute distance to the target level averaged across the NoNF (blue) and the NF runs (red) respectively. Middle panels: The group average absolute distance to the target level for each NF run separately. Lower panels: The group average absolute distance to the target level for each NoNF run separately.

Remarks: Error bars indicate the standard error of the mean.

Abbreviations: NoNF, No Neurofeedback run; NF, Neurofeedback run. 


\section{DISCUSSION}

The current study investigated whether it is possible to self-regulate the DMN bi-directionally to three different target levels with the help of rt-fMRI neurofeedback. Participants were able to modulate their activation level within the DMN (mainly including the two central hubs PCC/precuneus and MPFC/vACC) to predefined target levels by performing two different mental tasks, self-episodic memory recall in the up-regulation and mental calculation in the down-regulation session. Throughout both sessions, participants did not instantaneously benefit from the feedback information during the DMN self-regulation when comparing to the runs without feedback information present (compare absolute distance of DMN activation to target level in NoNF and NF runs, Fig. 6, upper panels). Furthermore, the activation and de-activation amplitude of the DMN in the up-regulation and down-regulation session respectively was higher within NoNF runs than in the NF runs (Fig.5, upper panels). Lastly however, the results suggest that the neurofeedback training induced learning within each session, as there was a difference from the first to the last run without neurofeedback (Fig. 6, lower panels).

\section{Reversal and training effects in the DMN up-regulation session}

Throughout the up-regulation session, two interesting descriptive effects could be observed across the runs, both on the group level as well as on the individual participant level. First, participants showed an initial, intuitive capability to self-regulate the DMN (first NoNF run, Fig.5A, middle panel). However, this capability was inverted with the presentation of the neurofeedback information in the first NF run. Notably, the self-regulation ability did not only decrease, but was even inverted and thereby resulting in a deactivation of the DMN. This visual neurofeedback input effect within the DMN can however be explained with the overall task-related deactivation of the DMN. While participants could focus on their self-regulation in the first NoNF run, they were presented with continuously updated feedback about their DMN activation in the NF runs. This likely resulted in an externally-triggered deactivation of the DMN and a consequently high difficulty for the participants to up-regulate the DMN within the NF runs. Second, participants could (on a descriptive level) at least partly overcome this visual 
neurofeedback input effect over the following NF runs (Fig.5A, middle panel) and learned to regulate the $\mathrm{DMN}$ in the positive direction. Taken together, the self-regulation possibility of the DMN was potentially hampered by the de-activating effect of the continuous visual neurofeedback input. While the described effects are not significant, they could contribute to the overall difficulty to self-regulate the DMN to the three target levels. As a consequence, future studies investigating neurofeedback applications within the DMN or trying to regulate input-sensitive regions should consider to use intermittent neurofeedback. In two recent studies, it has been found that intermittent neurofeedback was even more efficient for participants in the regulation process than continuous neurofeedback information (Hellrung et al., 2018; Johnson et al., 2012). Advantages of intermittent feedback are that it is robust against noise present in the data, and participants do not have to get used to the inherent delay of the BOLD signal. Another advantage might also apply to neurofeedback applications targeting the DMN as this version of feedback also overcomes the continuously changing aspect of the feedback, which seems disruptive in applications targeting the DMN.

\section{Delay of neurofeedback learning effect}

In both up- and down-regulation sessions, participants showed a difference in the gradual self-regulation ability from the first to the last NoNF run (Fig.6, lower panels) while differences across NF runs were not significant (Fig.6, middle panels). This suggests that neurofeedback induced some kind of learning without visually being detectable within the NF runs. This delayed neurofeedback learning effect was expressed in different manners in the up- and down-regulation sessions: While the participants showed a trend to improve their self-regulation in the down-regulation session (Fig. 5B, lower panel), they actually showed a decrease in self-regulation performance in the up-regulation session (significant if tested two-sided, Fig. 5A, lower panel). This degradation of the self-regulation capability was unexpected, as we hypothesised an increase in performance after neurofeedback. Furthermore, as the visual neurofeedback input was missing in the last NoNF run, this should have even boosted the activation levels to a higher degree as the DMN deactivating effect of the neurofeedback visual input was removed. From this, it seems that the neurofeedback training induced a kind of 
learning how to up-regulate the DMN when presented with neurofeedback, which however resulted in a lower performance when the feedback information was removed. The fact that these effects were present in the majority of participants (Fig. S1) clearly implies that future studies should investigate which specific mechanisms allowed this degradation of regulation performance to occur. One alternative explanation for the effect in the up-regulation session could have been a growing fatigue over the course of the scanning session. However, the gradual activation in the primary task region (hippocampus) across runs does not seem to explain this effect fully on the basis of fatigue. While there was a slight difference between the first and last NoNF run in this area (Fig.5A, lower panel), both runs show a task-positive, gradual modulation along the three target levels (Fig.5A, lower panel), suggesting that participants still actively engaged in self-episodic memory recall. Also in the IPS, there was a gradual activation in response to the three target levels during the down-regulation session, showing that participants continuously performed the task across the whole session (Fig.5B, lower panel). Taken together, the precise underlying mechanisms of the delayed learning effects in both up-and down-regulation of the DMN should be monitored closely in future studies as a decrease in self-regulation performance, even an inability to activate the DMN voluntarily, is not desired. Furthermore, follow-up tests should be made to see whether the changes within the neurofeedback training session last over time.

\section{Reflection on the mental strategies to regulate the DMN}

Research on $\mathrm{fMRI}$ neurofeedback has mainly investigated modulating the activation in brain regions whose functions are well defined and consequently, region-specific tasks could be used as modulation strategies. In case of the current study however, the specific functions of the DMN are not clearly defined which makes it difficult to find tasks primarily targeting the DMN. We further refrained from using meditation practices, mindfulness training or related strategies, as these have been shown to induce changes in breathing patterns or arterial and tissue oxygenation (Bernardi, Bordino, Bianchi, \& Bernardi, 2017; Melville, Chang, Colagiuri, Marshall, \& Cheema, 2012; Tang et al., 2009). As the fMRI signal depends on the oxygenation level in the blood, changes in breathing alone can result in changes of the signal (Kastrup, Krüger, Glover, Neumann-Haefelin, \& Moseley, 1999). To exclude any confounds 
related to this possibility, we chose self-episodic memory recall and mental calculation as the mental strategies to regulate the DMN in the up- and down-regulation session respectively. However, these strategies are not primarily targeting the DMN, and therefore might not have been optimal for the neurofeedback setting. As it is difficult to pinpoint specific strategies, future studies might want to not suggest any strategies to the participant and let them try to modulate the signal by finding a strategy themselves.

\section{CONCLUSION}

The results of this feasibility study suggest that there is only a limited possibility to gradually self-regulate the DMN bi-directionally with real-time fMRI neurofeedback, at least when providing moment-to-moment visual feedback. However, the current study emphasises important considerations in methodology when trying to regulate the DMN with fMRI neurofeedback. On the one hand, mental strategy selection should be revised to not narrowing down participants to one specific, potentially suboptimal task. On the other hand, neurofeedback applications with the DMN as a target network should use intermittent feedback to avoid visual input effects on the DMN regulation capability and thereby increasing the possibility of self-regulation success.

\section{ACKNOWLEDGEMENTS}

We would like to thank Armin Heinecke for his help with data analysis and Suvi Sundqvist for her help in organizational issues and data processing.

This work was supported by the Human Enhancement and Learning (HEaL) grant provided by Maastricht University, the Netherlands. 


\section{REFERENCES}

Andrews-Hanna, J. R., Reidler, J. S., Huang, C., \& Buckner, R. L. (2010). Evidence for the default network's role in spontaneous cognition. Journal of Neurophysiology, 104(1), 322-35. http://doi.org/10.1152/jn.00830.2009

Bernardi, N. F., Bordino, M., Bianchi, L., \& Bernardi, L. (2017). Acute fall and longterm rise in oxygen saturation in response to meditation. Psychophysiology, 54(12), 1951-1966. http://doi.org/10.1111/psyp.12972

Broyd, S. J., Demanuele, C., Debener, S., Helps, S. K., James, C. J., \& Sonuga-Barke, E. J. S. (2009). Default-mode brain dysfunction in mental disorders: A systematic review. Neuroscience \& Biobehavioral Reviews, 33(3), 279-296. http://doi.org/10.1016/J.NEUBIOREV.2008.09.002

Buckner, R. L., Andrews-Hanna, J. R., \& Schacter, D. L. (2008). The Brain's Default Network: Anatomy, Function, and Relevance to Disease. Annals of the New York Academy of Sciences, 1124(1), 1-38. http://doi.org/10.1196/annals.1440.011

Christoff, K., Gordon, A. M., Smallwood, J., Smith, R., \& Schooler, J. W. (2009). Experience sampling during $\mathrm{fMRI}$ reveals default network and executive system contributions to mind wandering. Proceedings of the National Academy of Sciences of the United States of America, 106(21), 8719-24. http://doi.org/10.1073/pnas.0900234106

Foster, B. L., Dastjerdi, M., \& Parvizi, J. (2012). Neural populations in human posteromedial cortex display opposing responses during memory and numerical processing. Proceedings of the National Academy of Sciences of the United States of America, 109(38), 15514-9. http://doi.org/10.1073/pnas.1206580109

Fox, M. D., Snyder, A. Z., Vincent, J. L., Corbetta, M., Van Essen, D. C., \& Raichle, M. E. (2005). The human brain is intrinsically organized into dynamic, anticorrelated functional networks. Proceedings of the National Academy of Sciences of the United States of America, 102(27), 9673-8. http://doi.org/10.1073/pnas.0504136102

Fransson, P. (2005). Spontaneous low-frequency BOLD signal fluctuations: An fMRI investigation of the resting-state default mode of brain function 
hypothesis. Human Brain Mapping, 26(1), 15-29. http://doi.org/10.1002/hbm.20113

Garrison, K. A., Santoyo, J. F., Davis, J. H., Thornhill, T. A., Kerr, C. E., \& Brewer, J. A. (2013). Effortless awareness: using real time neurofeedback to investigate correlates of posterior cingulate cortex activity in meditators' self-report. Frontiers in Human Neuroscience, 7, 440. http://doi.org/10.3389/fnhum.2013.00440

Garrison, K. A., Scheinost, D., Worhunsky, P. D., Elwafi, H. M., Thornhill, T. A., Thompson, E., ... Brewer, J. A. (2013). Real-time fMRI links subjective experience with brain activity during focused attention. Neurolmage, 81, 110-8. http://doi.org/10.1016/j.neuroimage.2013.05.030

Goebel, R., Sorger, B., Kaiser, J., Birbaumer, N., \& Weiskopf, N. (2004). BOLD brain pong: self-regulation of local brain activity during synchronously scanned, interacting subjects. In Society for Neuroscience, Washington, DC.

Harmelech, T., Friedman, D., \& Malach, R. (2015). Differential magnetic resonance neurofeedback modulations across extrinsic (visual) and intrinsic (defaultmode) nodes of the human cortex. The Journal of Neuroscience, 35(6), 2588-95. http://doi.org/10.1523/JNEUROSCI.3098-14.2015

Hellrung, L., Dietrich, A., Hollmann, M., Pleger, B., Kalberlah, C., Roggenhofer, E., ... Horstmann, A. (2018). Intermittent compared to continuous real-time fMRI neurofeedback boosts control over amygdala activation. Neurolmage, 166, 198-208. http://doi.org/10.1016/J.NEUROIMAGE.2017.10.031

Johnson, K. A., Hartwell, K., LeMatty, T., Borckardt, J., Morgan, P. S., Govindarajan, K., ... George, M. S. (2012). Intermittent "real-time" fMRI feedback is superior to continuous presentation for a motor imagery task: a pilot study. Journal of Neuroimaging, 22(1), 58-66. http://doi.org/10.1111/j.15526569.2010.00529.x

Kastrup, A., Krüger, G., Glover, G. H., Neumann-Haefelin, T., \& Moseley, M. E. (1999). Regional Variability of Cerebral Blood Oxygenation Response to Hypercapnia. Neurolmage, 10(6), 675-681. http://doi.org/10.1006/NIMG.1999.0505

Krause, F., Benjamins, C., Lührs, M., Eck, J., Noirhomme, Q., Rosenke, M., ... Goebel, R. (2017). Real-time fMRI-based self-regulation of brain activation across 
different visual feedback presentations. Brain-Computer Interfaces, 4(1-2), 87-101. http://doi.org/10.1080/2326263X.2017.1307096

Mason, M. F., Norton, M. I., Van Horn, J. D., Wegner, D. M., Grafton, S. T., \& Macrae, C. N. (2007). Wandering minds: the default network and stimulusindependent thought. Science, 315(5810), 393-5. http://doi.org/10.1126/science.1131295

Melville, G. W., Chang, D., Colagiuri, B., Marshall, P. W., \& Cheema, B. S. (2012). Fifteen minutes of chair-based yoga postures or guided meditation performed in the office can elicit a relaxation response. Evidence-Based Complementary and Alternative Medicine, 2012, 501986. http://doi.org/10.1155/2012/501986

Moeller, S., Yacoub, E., Olman, C. A., Auerbach, E., Strupp, J., Harel, N., \& Uğurbil, K. (2010). Multiband multislice GE-EPI at 7 tesla, with 16-fold acceleration using partial parallel imaging with application to high spatial and temporal whole-brain fMRI. Magnetic Resonance in Medicine, 63(5), 1144-53. http://doi.org/10.1002/mrm.22361

Mohan, A., Roberto, A. J., Mohan, A., Lorenzo, A., Jones, K., Carney, M. J., ... Lapidus, K. A. B. (2016). The Significance of the Default Mode Network (DMN) in Neurological and Neuropsychiatric Disorders: A Review. The Yale Journal of Biology and Medicine, 89(1), 49-57.

Raichle, M. E. (2015). The Brain's Default Mode Network. Annual Review of Neuroscience, 38, 433-447. http://doi.org/10.1146/annurev-neuro071013-014030

Scheibner, H. J., Bogler, C., Gleich, T., Haynes, J.-D., \& Bermpohl, F. (2017). Internal and external attention and the default mode network. Neurolmage, 148, 381-389. http://doi.org/10.1016/J.NEUROIMAGE.2017.01.044

Smallwood, J., \& Schooler, J. W. (2015). The Science of Mind Wandering: Empirically Navigating the Stream of Consciousness. Annual Review of Psychology, 66(1), 487-518. http://doi.org/10.1146/annurev-psych-010814-015331

Sorger, B., Kamp, T., Weiskopf, N., Peters, J. C., \& Goebel, R. (2018). When the brain takes 'BOLD' steps: Real-time fMRI neurofeedback can further enhance the ability to gradually self-regulate regional brain activation. Neuroscience, 378, 71-88. DOI: 10.1016/j.neuroscience.2016.09.026 
Spreng, R. N., \& Grady, C. L. (2010). Patterns of Brain Activity Supporting Autobiographical Memory, Prospection, and Theory of Mind, and Their Relationship to the Default Mode Network. Journal of Cognitive Neuroscience, 22(6), 1112-1123. http://doi.org/10.1162/jocn.2009.21282

Sulzer, J., Haller, S., Scharnowski, F., Weiskopf, N., Birbaumer, N., Blefari, M. L., ... Sitaram, R. (2013). Real-time fMRI neurofeedback: Progress and challenges. Neurolmage, 76, 386-399. http://doi.org/10.1016/J.NEUROIMAGE.2013.03.033

Tang, Y.-Y., Ma, Y., Fan, Y., Feng, H., Wang, J., Feng, S., ... Fan, M. (2009). Central and autonomic nervous system interaction is altered by short-term meditation. Proceedings of the National Academy of Sciences of the United States of America, 106(22), 8865-70. http://doi.org/10.1073/pnas.0904031106

Whitfield-Gabrieli, S., \& Ford, J. M. (2012). Default Mode Network Activity and Connectivity in Psychopathology. Annual Review of Clinical Psychology, 8, 49-76. http://doi.org/10.1146/annurev-clinpsy-032511-143049

Zhang, G., Zhang, H., Li, X., Zhao, X., Yao, L., \& Long, Z. (2013). Functional Alteration of the DMN by Learned Regulation of the PCC Using Real-Time fMRI. IEEE Transactions on Neural Systems and Rehabilitation Engineering, 21(4), 595-606. http://doi.org/10.1109/TNSRE.2012.2221480 


\section{SUPPLEMENTARY MATERIAL}
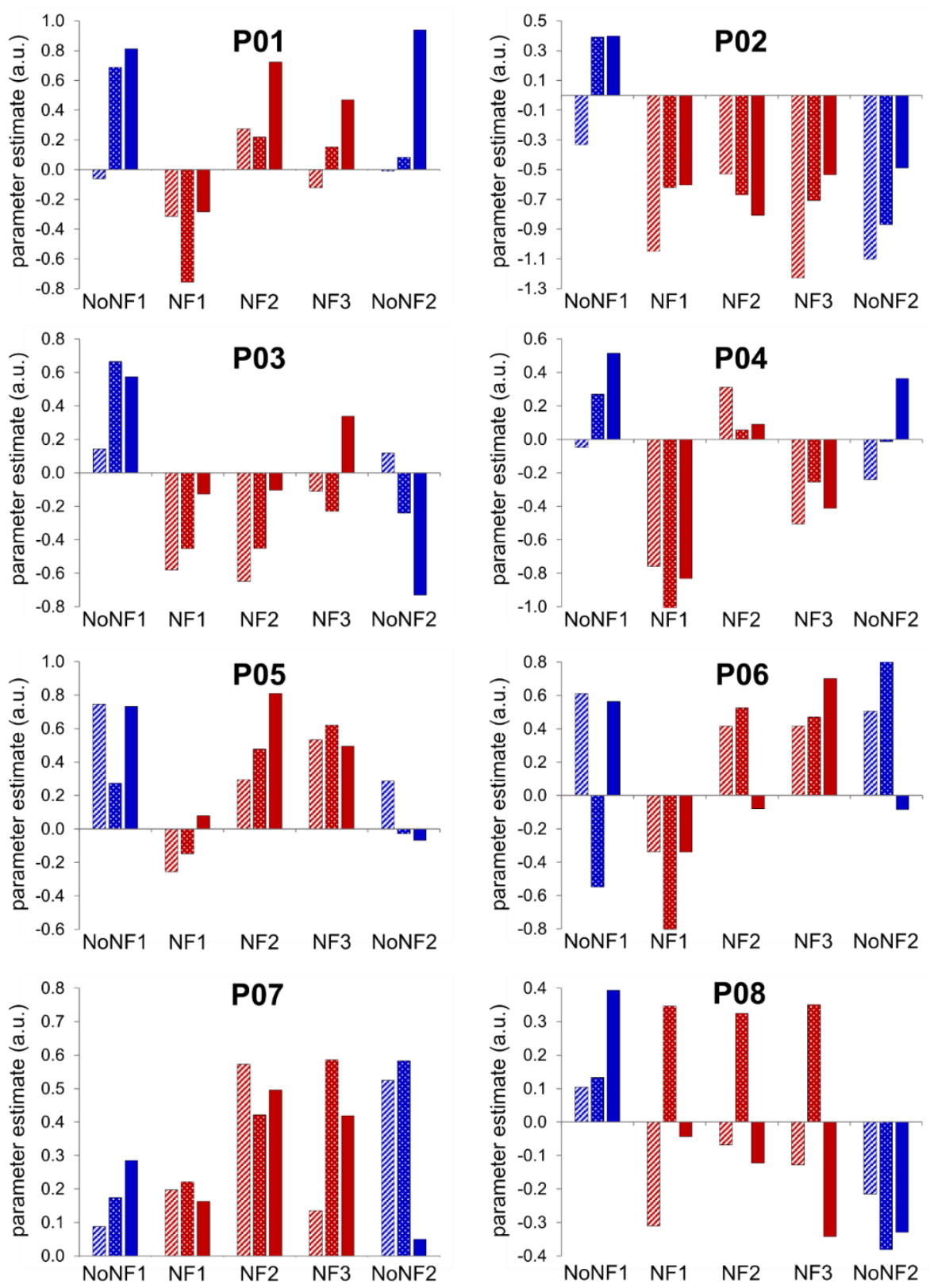

Fig.S1: Activation levels in the neurofeedback target DMN in individual participants in the upregulation session. The individual activation (beta values) within the neurofeedback target DMN $\rightarrow$ 
for the three target level conditions (stripes: low level; dots: medium level; filled: high level) is displayed averaged across the NoNF (blue) and the NF runs (red).

Abbreviations: a.u., arbitrary unit; NoNF, No Neurofeedback run; NF, Neurofeedback run. 

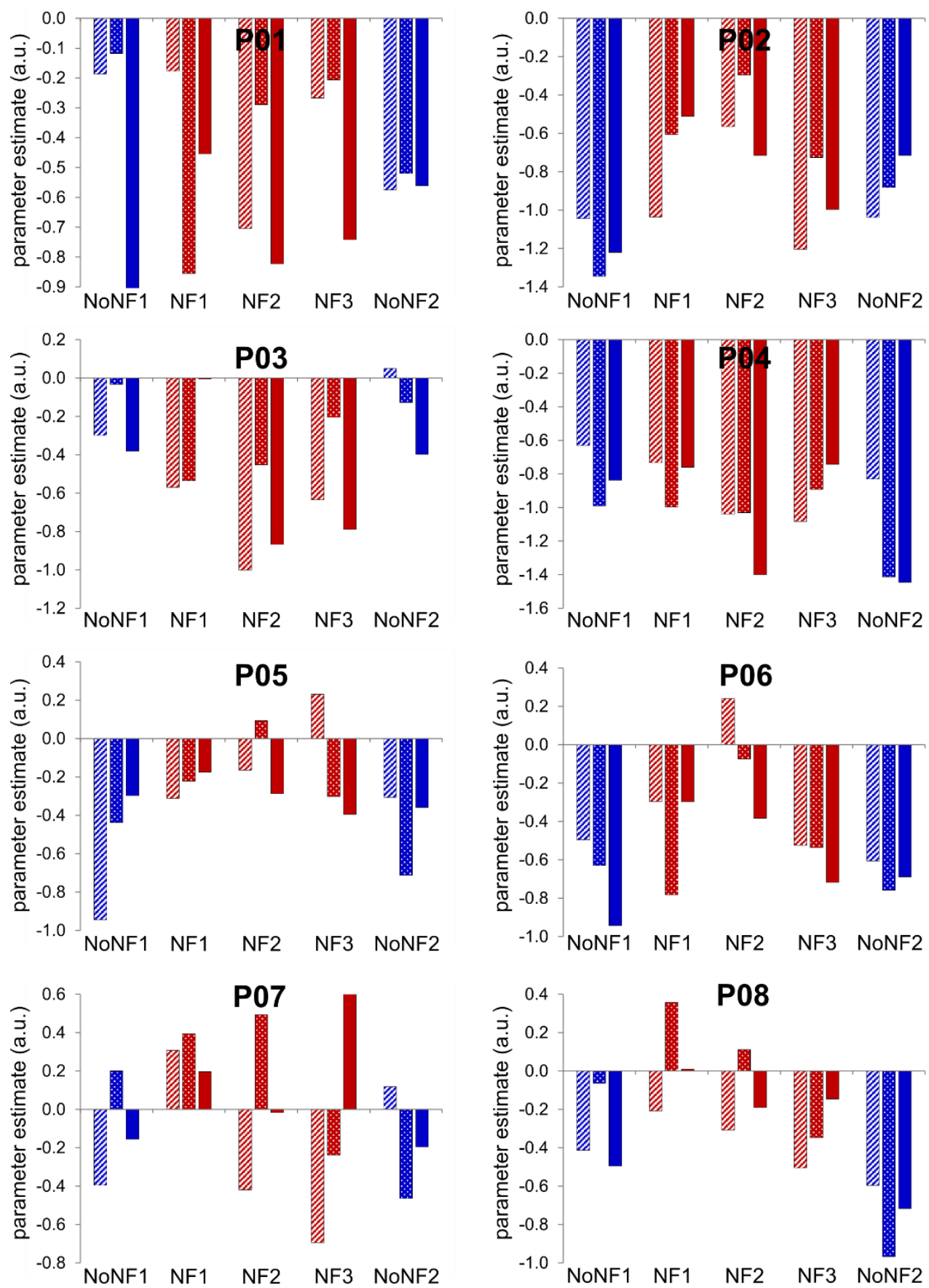
Fig.S2: Activation levels in the neurofeedback target DMN in individual participants in the downregulation session. The individual activation (beta values) within the neurofeedback target DMN for the three target level conditions (stripes: low level; dots: medium level; filled: high level) is displayed averaged across the NoNF (blue) and the NF runs (red).

Abbreviations: a.u., arbitrary unit; NoNF, No Neurofeedback run; NF, Neurofeedback run. 



\section{CHAPTER 5}

\section{SUMMARY AND GENERAL DISCUSSION}
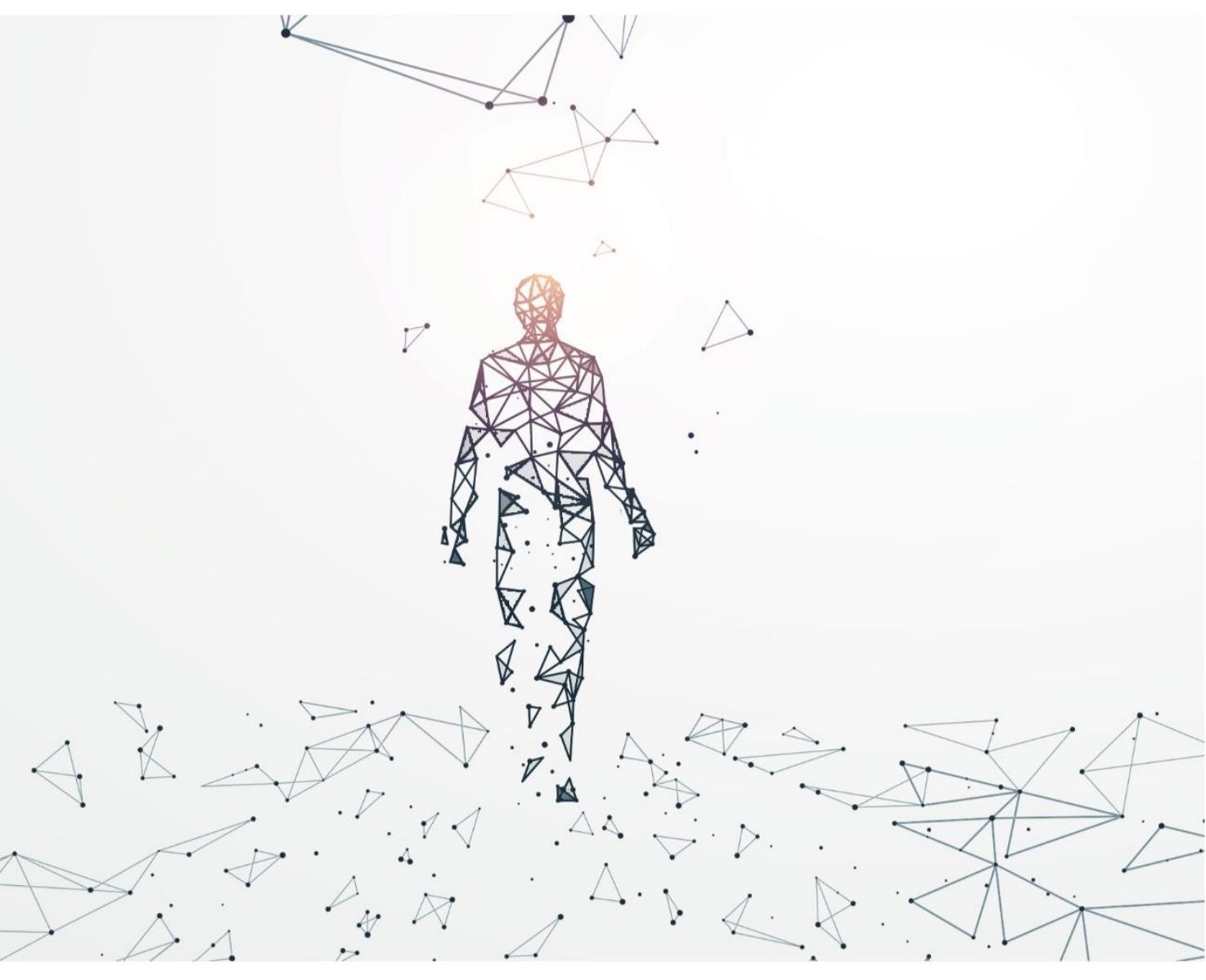

While research on cognitive enhancement has primarily used passive brain stimulation or psychopharmacological interventions in the past, a more active neurofeedback training approach could potentially enrich the spectrum of methods investigating cognitive enhancement without the side effects associated with medication or stimulation devices, alongside the possibility of more specifically targeting specific brain areas or networks. However, as rt-fMRI neurofeedback is a quite young research field and has primarily looked at restoring cognitive functioning in the past, it is not clear how far this technique can be used for improving cognition. Therefore, the aim of the current dissertation was to investigate the potential use of rt-fMRI neurofeedback for cognitive enhancement. The presented studies in this dissertation hereby followed a structured, stepwise approach with each study establishing feasibility for different methodological, as well as content-specific considerations on the strive for cognitive enhancement. The dissertation started with more methodological questions about using neurofeedback (Chapter 2), then advancing with the identification of a brain state which is beneficial for cognitive performance (Chapter $\mathbf{3}$ ), in order to finally conclude with a feasibility study of self-regulating the identified brain state with rt-fMRI neurofeedback (Chapter 4). In the following, the empirical studies included in this dissertation are first summarised, followed by an explanation of the implications of this work for different research fields. More specifically, methodological considerations for neurofeedback research, as well as considerations using neurofeedback to modulate the default mode network (DMN) will be presented. Moreover, specific learning mechanisms during neurofeedback will be discussed in light of the current dissertation, alongside ethical considerations for cognitive enhancement. The dissertation will conclude with describing current challenges and suggesting tasks for future research to advance the field of enhancing cognition with rt-fMRI neurofeedback.

\section{SUMMARY}

This dissertation includes a collection of three empirical studies, all aimed at establishing feasibility for a cognitive enhancement approach with rt-fMRI neurofeedback. More specifically, we first carried out a methodological investigation in Chapter $\mathbf{2}$ to look at whether rt-fMRI neurofeedback helps humans to specifically perform self-regulation to reach a certain brain state. In this study, 
participants underwent two MRI scanning sessions, one in which they were asked to modulate regional brain activation to three different, predefined target levels, and one in which they additionally received neurofeedback information about their current activation in the neurofeedback target region. The results of this study show that participants were able to achieve different brain activation levels purely on the basis of detailed instructions of the activation and modulation strategies. Moreover, it could be shown that when participants received additional neurofeedback information about the momentary activation level, they could perform even better, enhancing their gradual self-regulation ability with the help of neurofeedback further.

In the second investigation, we aimed at identifying a brain state which is beneficial for cognitive task performance and which could be used as a target brain state for a cognitive enhancement study. We examined whether a certain 'baseline' state of the DMN leads to better performance on a cognitive task (Chapter 3), as this network has been implicated in various mental disorders, as well as several cognitive functions. For this study, participants performed a mental rotation task in an extremely sparse event-related design, and we examined whether the pre-stimulus state of the DMN could predict subsequent task performance levels. The results suggest that a low pre-stimulus DMN activation preceded correct responses, while a low within-DMN connectivity predicted fast responses.

Based on the results of the first two studies (Chapter $2 \& 3$ ), we then combined the acquired knowledge and investigated whether participants could learn to control the DMN precisely with the help of neurofeedback. In this feasibility study, participants were asked to self-regulate their DMN activation bi-directionally to three different target levels (Chapter 4), accompanied by the visual presentation of rt-fMRI neurofeedback. For this, participants underwent two neurofeedback sessions, one which aimed at up-regulating the DMN, and one which targeted the down-regulation of the DMN to three predefined target levels. In each session, we instructed participants to perform a mental task for reaching the different target levels, suggesting self-episodic memory recall for the up-regulation session and mental calculation in the down-regulation session. In contrast to our findings in Chapter 2, participants could not benefit instantaneously from the neurofeedback information. However, it could be shown that participants learned from the neurofeedback training, as pre-/post-tests showed a difference in the 
self-regulation ability. Unexpectedly, the learning reversed the self-regulation ability in the up-regulation session, as participants showed a lower self-regulation ability after the neurofeedback training, which might be related to the difficulty to incorporate moment-to-moment feedback when targeting the DMN.

Combined, the empirical studies presented in this dissertation describe the different steps in the process of establishing feasibility for an rt-fMRI procedure studying cognitive enhancement. The studies provide a new paradigm for neurofeedback research, demonstrate novel insights in the contribution of the DMN in cognitive performance, and additionally raise new questions regarding learning mechanisms during neurofeedback. Taken together, the knowledge gained from these studies can be used to guide further studies in their strive for cognitive enhancement.

\section{CONSIDERATIONS FOR NEUROFEEDBACK PARADIGMS}

Since its development in 2003 (Weiskopf et al., 2003), rt-fMRI neurofeedback training is an increasingly popular field of research, not only because it fascinates researchers and participants to access and modulate brain activation at the conscious level, but also because it has a great potential in neuroscientific, as well as clinical or enhancement applications. As this research field is quite young (the pioneering study by deCharms and colleagues on rt-fMRI neurofeedback in chronic pain only dates back to 2005 (DeCharms et al., 2005)), there is still a demand for optimizing neurofeedback protocols. One question which is discussed in the neurofeedback field is whether and how to instruct participants for the neurofeedback training (Thibault, Lifshitz, \& Raz, 2016; Thibault, MacPherson, Lifshitz, Roth, \& Raz, 2018). Some studies use detailed instructions to describe how a brain region can be modulated, thus implementing strategies which are based on elaborated neuroscientific research. Also in the studies included in the current dissertation, we instructed participants with detailed descriptions of mental strategies they could use for the self-regulation. We could demonstrate that following these instructions alone already allows participants to gradually self-regulate their brain activation (Chapter 2), while providing neurofeedback information additionally improved this ability. Importantly, the neurofeedback target regions in this study comprised regions which are well-studied and which are driven reliably and unambiguously by the instructed strategies (e.g., Broca's area 
can be accessed and modulated by varying aspects of inner speech, such as speed). However, if neurofeedback training targets a region, a network, or an activation pattern, which cannot be modulated unambiguously with a pre-known strategy, it might be beneficial to apply a more liberal instruction or no instruction at all (e.g., Amano, Shibata, Kawato, Sasaki, \& Watanabe, 2016; Shibata, Watanabe, Sasaki, \& Kawato, 2011), allowing participants to freely explore which mental strategy drives the neurofeedback signal. Self-regulating the DMN with rt-fMRI neurofeedback might be an example where such an instruction approach suggesting to explore suitable strategies might be better suited: The instructed tasks in Chapter 4 (self-episodic memory for up-regulating, mental calculation for down-regulating the $\mathrm{DMN}$ ) regulate the $\mathrm{DMN}$, but there are several others, including mind wandering and daydreaming (Andrews-Hanna, Reidler, Huang, \& Buckner, 2010; Christoff, Gordon, Smallwood, Smith, \& Schooler, 2009; Lieberman, 2007; Mason et al., 2007; Scheibner, Bogler, Gleich, Haynes, \& Bermpohl, 2017; Smallwood \& Schooler, 2015). As participants were not able to gradually modulate the DMN during the neurofeedback training, it might be that in such a network-based neurofeedback approach with several tasks regulating the network, the instructions could be communicated more liberally, giving participants more room to explore for themselves which strategy works best.

Another consideration in neurofeedback studies involves the brain activation magnitude participants are requested to reach with self-regulation. Conventional neurofeedback studies ask participants to maximise or minimise their activation in the neurofeedback target region (Thibault et al., 2018). The gradual neurofeedback protocol investigated in Chapter 2 demonstrates a new paradigm which can be used to gain finer access to the functional mechanisms of a brain region. This paradigm could be used to access behavioural modification with neurofeedback in the context of cognitive enhancement. More specifically, it could be investigated whether different activation levels of the target region result in different behavioural outcome, by, for example, self-regulating the brain activation to a given level and subsequently presenting a task. If behavioural performance varied depending on the magnitude of self-regulation before the trial, it would shed new neuroscientific insights into the functional mechanisms of the neurofeedback target regions. In the case of the DMN, a certain DMN state could be induced (e.g., a low, a medium, and a high connectivity between the PCC and the MPFC with 
neurofeedback), followed by the presentation of a trial of the mental rotation task from Chapter 3. In a successful neuro-enhancement experiment, the brain state magnitude could then be related to the response speed. In this way, cognitive enhancement could be tested by directly accessing the behaviour through changing the brain states. In fact, previous research has already tried to modulate behaviour with rt-fMRI. In their adaptive rt-fMRI study, Yoo and colleagues monitored brain states online and presented to-be-learned items when participants were passing a 'good' or a 'bad' brain state for learning (Yoo et al., 2012). In a recognition test after the exposition to the stimuli, participants could remember those items presented in a 'good' brain state more accurately than when they were presented in a 'bad' brain state for learning. However, it remains to be tested whether we can also induce different kinds of brain states with rt-fMRI neurofeedback to modulate behaviour.

One important aspect of the gradual neurofeedback paradigm independent of study-specific factors is the potential motivational value of such a precise feedback task. It is conceivable that a successful gradual neurofeedback approach is more motivating and rewarding than the conventional maximizing/minimizing approach, as there are potentially more moments which could act rewarding given the different trial types. It nevertheless still needs to be evaluated whether a successful gradual neurofeedback training contributes to percept of increased control, in comparison to the conventional maximizing/minimizing approach. Especially for patient populations with anxiety disorders, such an approach would then potentially be powerful, as the feeling of internal control is diminished in these patient populations (Cloitre, Heimberg, Liebowitz, \& Gitow, 1992).

\section{ENHANCING COGNITION BY SELF-REGULATING THE DMN WITH RT-FMRI NEUROFEEDBACK}

The current dissertation aimed at establishing steps to enhance cognition by exemplarily studying the significance of the pre-stimulus DMN state for subsequent task performance (Chapter $\mathbf{3}$ ) and investigating its potential self-regulation with the help of rt-fMRI neurofeedback (Chapter 4). It has recently been suggested that the DMN fluctuates between two different states when participants are resting, an externally-oriented state characterised by a low activation of the DMN and an internally-oriented state, characterised by a high DMN activation (Fox et al., 2005; 
Fransson, 2005; Tian et al., 2007; Vanhaudenhuyse et al., 2011). The results of Chapter 3 extend previous evidence that a low DMN state is beneficial for cognitive performance and a high DMN state hampers it (Eichele et al., 2008; Li, Yan, Bergquist, \& Sinha, 2007; Soravia et al., 2016; Weissman, Roberts, Visscher, \& Woldorff, 2006). Following this line, we tried to induce different DMN states with rt-fMRI neurofeedback. However, participants could not instantaneously use the neurofeedback information to reach the different target levels. Before future studies can investigate whether an induced DMN state can predict subsequent performance on a cognitive task, it first needs to be clarified whether we can achieve instantaneous self-regulation on a single trial basis. In order to do so, several changes in design might be advantageous, for example changing the mental strategy to an exploratory approach (see previous section), only focusing on one modulation direction and introducing more neurofeedback training.

\section{STUDY-SPECIFIC LEARNING PROCESSES IN NEUROFEEDBACK \& ETHICAL CONSIDERATIONS}

What is learned during neurofeedback? A common idea is that operant conditioning plays a major role in learning from the neurofeedback training (Sulzer et al., 2013) and neurofeedback success is associated with a delayed striatum response (Skottnik, Sorger, Kamp, Linden, \& Goebel, submitted). A clear description of the learning mechanisms has, however, not been stated. The findings of the study on the DMN self-regulation with rt-fMRI neurofeedback (Chapter 4) describe a kind of learning which is unusual for neurofeedback applications. Throughout the up- and down-regulation session, participants did not seem to benefit instantaneously from the neurofeedback information to reach the three different target levels. However, their brain activation within the DMN changed after the neurofeedback training. More specifically, participants improved their gradual self-regulation ability in the down-regulation session after the neurofeedback training, although this could not be deducted from the activation during the neurofeedback training. In contrast, participants performed worse after the training in the up-regulation session, while during the neurofeedback training, such a worsening of performance was not predictable. Instead, after their performance initially dropped significantly in the first neurofeedback run compared to the first run without neurofeedback, their self-regulation ability improved across 
subsequent neurofeedback runs. The difficulty to up-regulate the DMN during neurofeedback training might be due to the visual input of the neurofeedback, which presumably de-activated the DMN. Overcoming this de-activation within the neurofeedback training was likely a difficult task for the participants and future studies should consider using intermittent feedback to circumvent these issues when regulating the $\mathrm{DMN}$.

As described above, participants showed self-regulation changes from pre- to post-test in both up- and down-regulation sessions, while no such effects could be seen in the neurofeedback runs. This suggests that even if participants did not perform well with regard to the brain activation levels during the neurofeedback training, it is conceivable that they either adapted their self-regulation strategy (un-) consciously, or the neurofeedback target region adapted according to the training. Thus, participants might be trained in something we do not have direct access to and which is not necessarily what we aim training them on. This raises some practical, as well as ethical questions with regard to neurofeedback applications for cognitive enhancement and emphasises the caution and diligence with which research should be performed. First, before we are training specific brain states with rt-fMRI neurofeedback, we should be sure what these brain states affect. For example, it remains unknown how overly training participants in achieving one direction of the DMN state (e.g., the down-regulation approach) affects their capacities on other cognitive functions, such as creativity. Previously, it has been shown that creativity is linked to an active DMN (Beaty et al., 2014) and it is not clear what a trained down-regulation might result in. Second, we need to be sure what our neurofeedback training induces. As it was shown in the results for the up-regulation session in Chapter 4, participants showed a decline in self-regulation performance after the training with rt-fMRI neurofeedback. Although the sample size was small, this effect could be seen in the majority of individual participants. Thus, we as researchers should carefully look at individual runs to potentially identify training effects which otherwise would have remained undetected and collect self-reports from the participants about potential motivational issues.

The abovementioned practical and ethical considerations are all specific to neurofeedback applications. However, this dissertation also needs to discuss general ethical considerations about cognitive enhancement when trying to 
investigate neurofeedback as a suited method for it. While neurofeedback needs to consider ethical questions such as those described above, it is a mental training approach with feedback from a neuroimaging method and in this way being similar to other mental trainings, such as mnemonics or training guided by apps. Therefore, several ethical considerations (e.g. concerning health) are not shared with other cognitive enhancement approaches. With respect to psychopharmacological and brain stimulation methods to enhance cognition, there has been an elaborate scientific discourse about whether or not it is morally justifiable to enhance humans in their functioning. Safety and health concerns are one of the greatest issues in the debate of cognitive enhancers. Especially for psychopharmacological cognitive enhancers, potential addictions, administration risks, as well as adverse events or side effects are increasingly discussed (Dresler et al., 2013; Farah, Smith, Ilieva, \& Hamilton, 2014). Independent of which method is used for studying cognitive enhancement, it remains the responsibility of the researchers as well as with ethical commissions to assess the safety of participants, as well as potential users. Safety is therefore to be prioritised in any neuro-enhancement application and any safety concerns should also be communicated by the responsible researchers. Also within this discussion on using rt-fMRI neurofeedback for cognitive enhancement, it was drawn attention to possible undesired learning mechanisms. Generally, however, the doubts and apprehensions about cognitive enhancement deal with potential futuristic scenarios if cognitive enhancement was found to achieve large effect sizes. Opponents are afraid that a higher societal desire for cognitive enhancement could potentially force people into engaging in it, for example if the application for a job would become more successful if the applicant was willing to enhance his or her cognitive capacities (Dresler et al., 2013). Evidently, it was shown that people would have a preference for flying with a certain company or go to a certain hospital if they knew the staff members took cognitive enhancers to increase attention (Chatterjee, 2004). Opponents further apprehend a potential inequality in society in case neuro-enhancement methods became economically profitable. However, the latter two fears are subject for speculation as effect sizes from any of the methods described above are so far quite small. In fact, research on cognitive enhancement has provided promising results, could however not establish consistent effects and therefore remains experimental (Allen \& Strand, 2015; Dresler et al., 2013; Farah et al., 2014). Moreover, even if cognitive enhancement 
became efficient and accessible, governmental regulations should protect the individual from inequality caused by cognitive enhancers. In fact, the US Presidential Commission for the Study of Bioethical Issues (Bioethics Commission) has recognised the ethical concerns and already published recommendations for studying the use and efficiency of neuro-enhancers ethically (Allen \& Strand, 2015). In conclusion, research on cognitive enhancement needs to be concerned with ethical questions and potential outcomes, especially to protect the individual from potential harm or safety concerns. An overly concern about dystopian settings is however not helpful in establishing and fostering an environment for safe and novel research.

\section{CONCLUSION, FUTURE OUTLOOK \& CHALLENGES}

This dissertation aimed at establishing the grounds for using rt-fMRI neurofeedback as a tool for cognitive enhancement. Rt-fMRI demonstrates a powerful potential to investigate neuroscientific questions, restoring cognitive functions, as well as enhancing performance. The presented dissertation on the one hand provides evidence which might lead to cognitive enhancement studies with rt-fMRI neurofeedback, on the other hand also points to the challenges which research in this field faces. The individual studies introduced new paradigms for neurofeedback applications, provided new evidence for the cognitive functions of the DMN and investigated the capacity to self-regulate this network with rt-fMRI neurofeedback. In order to advance the strive for cognitive enhancement with this technique, several steps and challenges for any enhancement application need to be considered:

1. It should be clearly formulated which cognitive function is targeted by the enhancement and the associated brain states or target regions should be clearly identifiable.

2. The targeted brain state should be examined regarding controllability with rt-fMRI neurofeedback.

3. It should be investigated in how far the gradual modulation of the targeted brain state or brain region results in related behavioural changes. 
4. It should be investigated whether neurofeedback training results in a transferrable ability to self-regulate the targeted brain state outside of the enhancement context and further

5. whether this induced self-regulation of the brain state has a beneficial and desired influence on behaviour.

All of these steps point towards a long and exciting journey on the way to establishing effective cognitive enhancement with rt-fMRI neurofeedback, but also pose challenges for neurofeedback approaches in the clinical context. However, when these challenges are overcome, it will become evident whether there is a future for rt-fMRI neurofeedback for cognitive enhancement, as well as in the clinical setting. 


\section{REFERENCES}

Allen, A. L., \& Strand, N. K. (2015). Cognitive Enhancement and Beyond: Recommendations from the Bioethics Commission. Trends in Cognitive Sciences, 19(10), 549-551. http://doi.org/10.1016/J.TICS.2015.08.001

Amano, K., Shibata, K., Kawato, M., Sasaki, Y., \& Watanabe, T. (2016). Learning to Associate Orientation with Color in Early Visual Areas by Associative Decoded fMRI Neurofeedback. Current Biology, 26(14), 1861-1866. http://doi.org/10.1016/j.cub.2016.05.014

Andrews-Hanna, J. R., Reidler, J. S., Huang, C., \& Buckner, R. L. (2010). Evidence for the default network's role in spontaneous cognition. Journal of Neurophysiology, 104(1), 322-35. http://doi.org/10.1152/jn.00830.2009

Beaty, R. E., Benedek, M., Wilkins, R. W., Jauk, E., Fink, A., Silvia, P. J., ... Neubauer, A. C. (2014). Creativity and the default network: A functional connectivity analysis of the creative brain at rest. Neuropsychologia, 64, 92-98. http://doi.org/10.1016/J.NEUROPSYCHOLOGIA.2014.09.019

Chatterjee, A. (2004). Cosmetic neurology: the controversy over enhancing movement, mentation, and mood. Neurology, 63(6), 968-74. http://doi.org/10.1212/01.WNL.0000138438.88589.7C

Christoff, K., Gordon, A. M., Smallwood, J., Smith, R., \& Schooler, J. W. (2009). Experience sampling during $\mathrm{fMRI}$ reveals default network and executive system contributions to mind wandering. Proceedings of the National Academy of Sciences of the United States of America, 106(21), 8719-24. http://doi.org/10.1073/pnas.0900234106

Cloitre, M., Heimberg, R. G., Liebowitz, M. R., \& Gitow, A. (1992). Perceptions of control in panic disorder and social phobia. Cognitive Therapy and Research, 16(5), 569-577. http://doi.org/10.1007/BF01175142

DeCharms, R. C., Maeda, F., Glover, G. H., Ludlow, D., Pauly, J. M., Soneji, D., ... Mackey, S. C. (2005). Control over brain activation and pain learned by using real-time functional MRI. Proceedings of the National Academy of Sciences of the United States of America, 102(51), 18626-31. http://doi.org/10.1073/pnas.0505210102

Dresler, M., Sandberg, A., Ohla, K., Bublitz, C., Trenado, C., Mroczko-Wąsowicz, A., ... Repantis, D. (2013). Non-pharmacological cognitive enhancement. 
Neuropharmacology,

http://doi.org/10.1016/J.NEUROPHARM.2012.07.002

Eichele, T., Debener, S., Calhoun, V. D., Specht, K., Engel, A. K., Hugdahl, K., ... Ullsperger, M. (2008). Prediction of human errors by maladaptive changes in event-related brain networks. Proceedings of the National Academy of Sciences of the United States of America, 105(16), 6173-8. http://doi.org/10.1073/pnas.0708965105

Farah, M. J., Smith, M. E., Ilieva, I., \& Hamilton, R. H. (2014). Cognitive enhancement. Wiley Interdisciplinary Reviews: Cognitive Science, 5(1), 95103. http://doi.org/10.1002/wcs.1250

Fox, M. D., Snyder, A. Z., Vincent, J. L., Corbetta, M., Van Essen, D. C., \& Raichle, M. E. (2005). The human brain is intrinsically organized into dynamic, anticorrelated functional networks. Proceedings of the National Academy of Sciences of the United States of America, 102(27), 9673-8. http://doi.org/10.1073/pnas.0504136102

Fransson, P. (2005). Spontaneous low-frequency BOLD signal fluctuations: An fMRI investigation of the resting-state default mode of brain function hypothesis. Human Brain Mapping, 26(1), 15-29. http://doi.org/10.1002/hbm.20113

Li, C.-S. R., Yan, P., Bergquist, K. L., \& Sinha, R. (2007). Greater activation of the "default" brain regions predicts stop signal errors. Neurolmage, 38(3), 640648. http://doi.org/10.1016/J.NEUROIMAGE.2007.07.021

Lieberman, M. D. (2007). Social Cognitive Neuroscience: A Review of Core Processes. Annual Review of Psychology, 58(1), 259-289. http://doi.org/10.1146/annurev.psych.58.110405.085654

Mason, M. F., Norton, M. I., Van Horn, J. D., Wegner, D. M., Grafton, S. T., \& Macrae, C. N. (2007). Wandering minds: the default network and stimulusindependent thought. Science, 315(5810), 393-5. http://doi.org/10.1126/science.1131295

Scheibner, H. J., Bogler, C., Gleich, T., Haynes, J.-D., \& Bermpohl, F. (2017). Internal and external attention and the default mode network. Neurolmage, 148, 381-389. http://doi.org/10.1016/J.NEUROIMAGE.2017.01.044 
Shibata, K., Watanabe, T., Sasaki, Y., \& Kawato, M. (2011). Perceptual learning incepted by decoded fMRI neurofeedback without stimulus presentation. Science, 334(6061), 1413-5. http://doi.org/10.1126/science.1212003

Skottnik, L., Sorger, B., Kamp, T., Linden, D. E. J., \& Goebel, R. (submitted). Success and failure of controlling the $\mathrm{fMRI}$-neurofeedback signal are reflected in the striatum.

Smallwood, J., \& Schooler, J. W. (2015). The Science of Mind Wandering: Empirically Navigating the Stream of Consciousness. Annual Review of Psychology, 66(1), 487-518. http://doi.org/10.1146/annurev-psych-010814-015331

Soravia, L. M., Witmer, J. S., Schwab, S., Nakataki, M., Dierks, T., Wiest, R., ... Jann, K. (2016). Prestimulus default mode activity influences depth of processing and recognition in an emotional memory task. Human Brain Mapping, 37(3), 924-932. http://doi.org/10.1002/hbm.23076

Sulzer, J., Haller, S., Scharnowski, F., Weiskopf, N., Birbaumer, N., Blefari, M. L., ... Sitaram, R. (2013). Real-time fMRI neurofeedback: Progress and challenges. Neurolmage, 76, 386-399. http://doi.org/10.1016/J.NEUROIMAGE.2013.03.033

Thibault, R. T., Lifshitz, M., \& Raz, A. (2016). The self-regulating brain and neurofeedback: Experimental science and clinical promise. Cortex, 74, 247261. http://doi.org/10.1016/J.CORTEX.2015.10.024

Thibault, R. T., MacPherson, A., Lifshitz, M., Roth, R. R., \& Raz, A. (2018). Neurofeedback with fMRI: A critical systematic review. Neurolmage, 172, 786-807. http://doi.org/10.1016/J.NEUROIMAGE.2017.12.071

Tian, L., Jiang, T., Liu, Y., Yu, C., Wang, K., Zhou, Y., ... Li, K. (2007). The relationship within and between the extrinsic and intrinsic systems indicated by resting state correlational patterns of sensory cortices. Neurolmage, 36(3), 684690. http://doi.org/10.1016/J.NEUROIMAGE.2007.03.044

Vanhaudenhuyse, A., Demertzi, A., Schabus, M., Noirhomme, Q., Bredart, S., Boly, M., ... Laureys, S. (2011). Two Distinct Neuronal Networks Mediate the Awareness of Environment and of Self. Journal of Cognitive Neuroscience, 23(3), 570-578. http://doi.org/10.1162/jocn.2010.21488

Weiskopf, N., Veit, R., Erb, M., Mathiak, K., Grodd, W., Goebel, R., \& Birbaumer, N. (2003). Physiological self-regulation of regional brain activity using real- 
time functional magnetic resonance imaging (fMRI): methodology and exemplary data. Neurolmage, 19(3), 577-86.

Weissman, D. H., Roberts, K. C., Visscher, K. M., \& Woldorff, M. G. (2006). The neural bases of momentary lapses in attention. Nature Neuroscience, $9(7)$, 971-978. http://doi.org/10.1038/nn1727

Yoo, J. J., Hinds, O., Ofen, N., Thompson, T. W., Whitfield-Gabrieli, S., Triantafyllou, C., \& Gabrieli, J. D. E. (2012). When the brain is prepared to learn: enhancing human learning using real-time fMRI. Neurolmage, 59(1), 84652. http://doi.org/10.1016/j.neuroimage.2011.07.063 



\section{APPENDIX \\ KNOWLEDGE VALORISATION ACKNOWLEDGEMENTS CURRICULUM VITAE PUBLICATIONS}
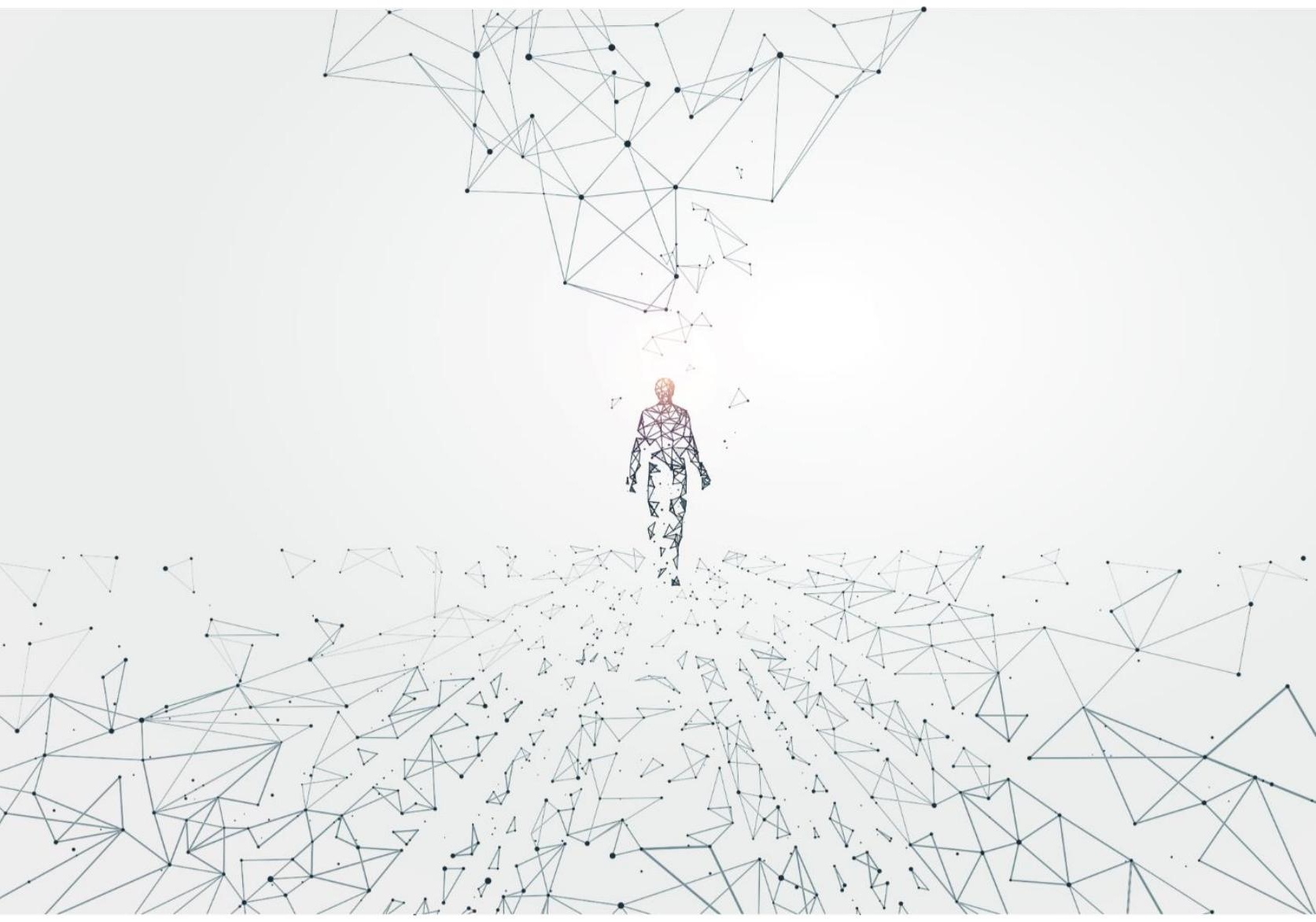



\section{KNOWLEDGE VALORISATION}

Within academia, the importance of knowledge valorisation gains an increasingly important significance. According to the National Valorisation Committee (2011), knowledge valorisation aims at

"creating value from knowledge, by making knowledge suitable and/or available for social (and/or economic) use and by making knowledge suitable for translation into competitive products, services, processes and new commercial activities" (Regulations governing the attainment of doctoral degree, UM, App. 4, §23).

On the one hand, engaging in knowledge valorisation is certainly an important aspect for the scientific community, as the experimental ideas themselves or the results of scientific endeavours often target societal, economic or governmental interests, whether directly or indirectly. In order to bridge the gap between academic work and these interests from outside academia, it is therefore necessary to translate the knowledge gained from science. However, and on the other hand, this is not an easy task and might not be directly applicable for all kinds of research, e.g. very fundamental research does not necessarily lead to any direct value for the economic market or societal wealth, but rather enriches the scientific community in order to increase our general understanding of the world, which might in itself again lead to benefits in social or economic matters, only on the long run. Therefore, writing a chapter about knowledge valorisation itself is a challenging and important task.

First of all, for the translation of knowledge, inter-disciplinary exchange is an important tool. Through this exchange, knowledge can propagate, creativity is facilitated and new research ideas can be sparked. The current thesis was part of a collaborative project called 'Human Enhancement and Learning" (HEaL), which focused on joining the fields of neuroscience and economics of education in order to combine their inherent expertise to gain new knowledge about human learning and improve education. To foster collaboration, as well as dialogue among these different fields, various meetings of the different research teams have been initiated throughout the different stages of the projects in this dissertation. 
Through these meetings, mutual knowledge dissemination was encouraged and led to fruitful research ideas.

As for the current dissertation, the experimental results can be valorised in different terms, mainly providing suggestions and guidelines for future experimental research on cognitive enhancement with rt-fMRI neurofeedback, but also being relevant for the educational, as well as health-care sector.

The main goal of this thesis was to establish feasibility of employing rt-fMRI neurofeedback for cognitive enhancement purposes. Throughout the dissertation, different stages for potential cognitive enhancement applications were described which can be used as guidelines for future research on this topic, including methodological considerations about neurofeedback research, identifying brain states which can be enhanced and establishing feasibility to enhance these brain states (Fig. 1). However, these steps are only the first ones in a series of measures to take for applying rt-fMRI neurofeedback for cognitive enhancement. Fig. 1 emphasises a more complete view of the different stages along this way. After establishing replicated results about the steps addressed in this thesis, it needs to be clarified whether there is indeed a behavioural benefit from cognitive enhancement with rt-fMRI neurofeedback. Only once all of these steps have been proven to be replicable, a translation to educational and health-care settings is feasible. The coming years will thus show whether it is really feasible to enhance cognition on the basis of rt-fMRI neurofeedback. 


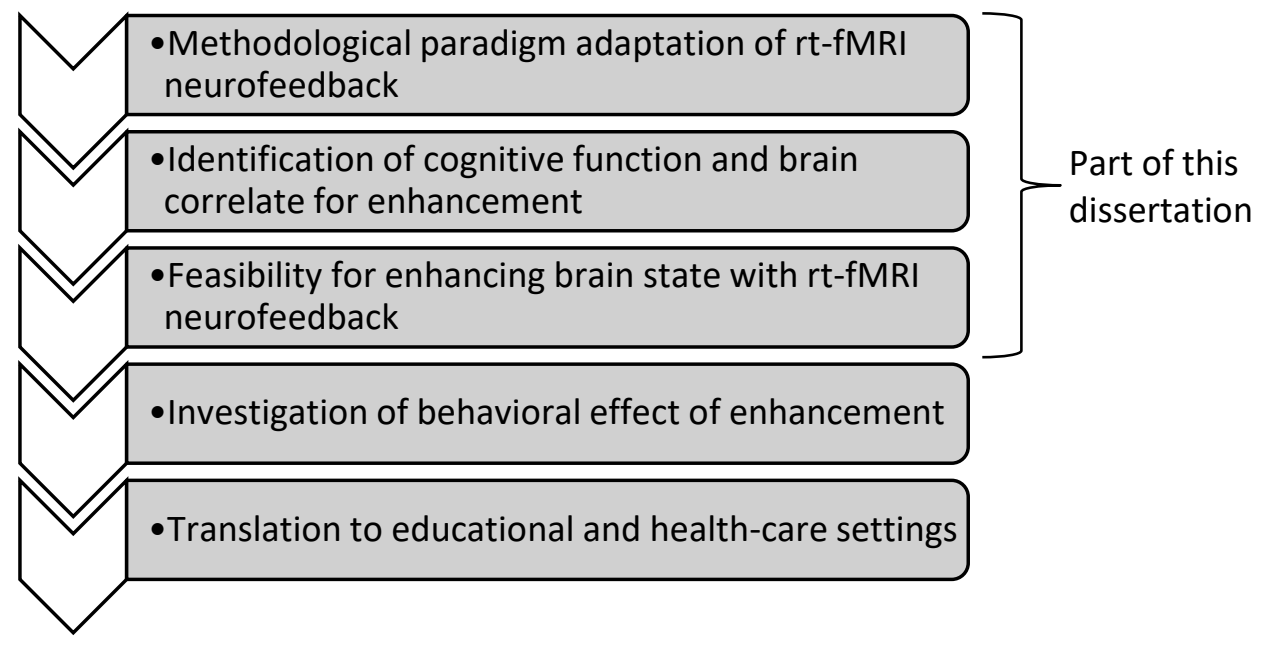

Fig. 1. Different stages in the process of studying cognitive enhancement with rt-fMRI neurofeedback.

One could argue that the work presented in this dissertation has a strong translational character, as it combines neuroscientific knowledge with applications for, e.g. the educational sector. However, all the subsequently mentioned aspects are only true once rt-fMRI neurofeedback fulfils all the criteria listed above. If so, neurofeedback could, in principle, be used in order to facilitate learning in schools or universities. In that sense, rt-fMRI neurofeedback could be used as an add-on learning medium in order to tailor every individual's learning strategies and maximise their efficiency or overcome pitfalls. Furthermore, health-care might benefit from the knowledge as rt-fMRI might constitute an alternative for pharmaceutical cognitive enhancers. However, the specific methods presented here are unlikely of any practical value for a broad application. Even if future studies on rt-fMRI neurofeedback for cognitive enhancement would result in robust, positive effects, it still remains highly questionable whether this method would find its way into practice for training or enhancing cognition over several scanning sessions, as the costs of employing an MRI scanner are high and machines are not readily and widely available. It is more conceivable to translate the findings into cheaper alternatives. It would, for example, be a possibility to tailor mental strategies based on research on cognitive enhancement to find robust strategies to alter specific brain states across people. With this knowledge, people could train 
the mental strategies without the necessity of undergoing rt-fMRI neurofeedback training. Another possibility would be to translate the knowledge acquired with rtfMRI neurofeedback into more portable solutions, as for example functional nearinfrared spectroscopy (fNIRS) or electroencephalography (EEG), to make it more accessible and cheaper to use.

Despite all of the abovementioned possibilities, the ultimate realistic goal of the research on cognitive enhancement might be different than the direct application: It might constitute one small piece of the puzzle as to how the human brain works and how we can influence it. Thereby, the message of this research might also be to incorporate the results from cognitive enhancement studies into models of human information processing to pave a way to a different understanding of human functioning and learning. After all, it should not be forgotten that the purest idea behind research may lie beyond directly accessible worth, as Seneca (c. 4 BC-AD 65, Roman philosopher) already pointed out in Naturales quaestiones:

"The time will come when diligent research over long periods will bring to light things which now lie hidden. A single lifetime, even though entirely devoted to the sky, would not be enough for the investigation of so vast a subject... And so this knowledge will be unfolded only through long successive ages. There will come a time when our descendants will be amazed that we did not know things that are so plain to them... Many discoveries are reserved for ages still to come, when memory of us will have been effaced." 
A P PENDIX

174 


\section{ACKNOWLEDGEMENTS}

This dissertation put into words what I have scientifically achieved in the past years. However, the work presented here would not exist without the scientific, inspirational, caring and motivating help of my fellow colleagues, friends and family. There are no words which describe my gratitude to the fullest. Nevertheless, I aim at expressing this gratitude in these acknowledgements.

First of all, I would like to thank my supervisors. Rainer, your devotion to science was inspirational from your first lecture I attended during my master programme. Even more importantly, your never-ending optimism and your kind character always impressed me. Bettina, you were a companion for my academic career from early on in the master programme and you supported me ever since. Your diligence and curious character are incomparable and I thank you for your support!

Amaia and Laurien, I value your help as paranymphs and smart, 'sciency' women throughout the defence and the preceding period a lot and I am happy to have such caring and supportive friends around me. Laurien, you are a lovely, caring and crazy person and I am looking back at so many amazing talks and discussions about science, psychotherapy and everyday life with all its challenges ('Just DO it, nothing is impossible!') and future possibilities throughout my PhD. Thank you for your funny and kind nature in the office and for being my personal provider of happiness in all kinds of ways! Amaiatje, your benevolence and warm-heartedness is exceptional, you always spread a warm atmosphere when you are around. It was so much fun to travel to Canada with you and I always valued your help and opinion on scientific problems.

I would especially like to thank my Aachen-Maastricht 'car gang'. Valentin, Helen, Michael, Arne \& Sanae, without you, I would have literally not made my way to this PhD. Thank you for the 'Starthilfe', meant in a literal (when I again forgot to switch off my headlights, right Valentin?) and in a more figurative way at the beginning of my PhD. I enjoyed our car rides together a lot (thanks for waiting for me so many 
times!), with all your good advices and all those talks about science, sports, cooking, and everyday life. Thank you for your friendship!

Thank you Hannah for your understanding and straight character, I value it a lot! It was lovely sharing the office with you who were the realist in our triplet of office mates, at the same time being supportive and caring. We definitely needed it!

I will always remember my amazing OXF55 lunch group with Hannah, Laurien, Amaia, Shruti and Anita. These were not only the best, most delicious meals I had, but especially the funniest and most intense lunch breaks. I miss them a lot and it was hard to say 'Bye now'!

I would like to thank all colleagues at the Cognitive Neuroscience department and Brain Innovation for the constructive, friendly and supportive atmosphere at work. I appreciate all the warmth and conversations there! Riny en Christl, ik ben heel blij dat jullie als grote hulp aan mijn zijde stonden tijdens mijn PhD. Ik vind het heel leuk, dat ik tenminste met jullie nog steeds Nederlands spreek! :) Our $\mathrm{BCl}$ group meetings with Bettina, Michael, Hannah, Laurien, Amaia, Leon, Deni and all the students were a help to structure my projects and to keep me on track. Thanks to all of you for your comments and questions throughout the projects!

On a more personal note, I would like to thank people outside the $\mathrm{CN}$ world at Maastricht University for supporting me, also my friends who helped me a lot in the process of the PhD by motivating and supporting me. One special 'thank you' goes to Sabine for your understanding, constructive and emotional support throughout our bachelor, master and PhD. You followed every step and I am happy that I had such a good friend by my side!

Der größte Dank gilt meiner Familie, die mich trägt und fördert.

Fabian und Raphael, ich bin dankbar, in eurer Mitte zu stehen und mit euch als Brüder die Welt gemeinsam entdeckt zu haben. Es ist schön, euch gemeinsam mit Lea, Ada und Marina bei so vielen wichtigen Momenten in meinem Leben an meiner Seite zu wissen. 
Mama und Papa, ihr seid meine Felsen in der Brandung, die mich immer bedingungslos unterstützt haben und deren Halt ich stets spüre. Ohne eure Motivation, euren Zuspruch und euer Vertrauen in mich hätte ich diesen Weg nicht gehen können.

Georg, ich danke dir für deinen Entdeckergeist und deine Begleitung in meinem Leben. Deine wisschenschaftliche Begeisterung haben mich in den letzten Monaten immens motiviert und haben einen großen Teil zu dieser Dissertation beigetragen (und auch dein Gehirn (:)). Dein offenes Ohr, deine Leichtigkeit und deine Fürsorge geben mir stets Kraft und lassen mich wachsen. 
A P PENDIX

178 


\section{CURRICULUM VITAE}

Tabea Kamp was born in Eschweiler, Germany, on $22^{\text {nd }}$ March 1989. She graduated from Städtisches Gymnasium Eschweiler in 2008, where she also received the Peter-Liesen Preis for social commitment at her school. She started studying Psychology at the Faculty of Psychology and Neuroscience, Maastricht

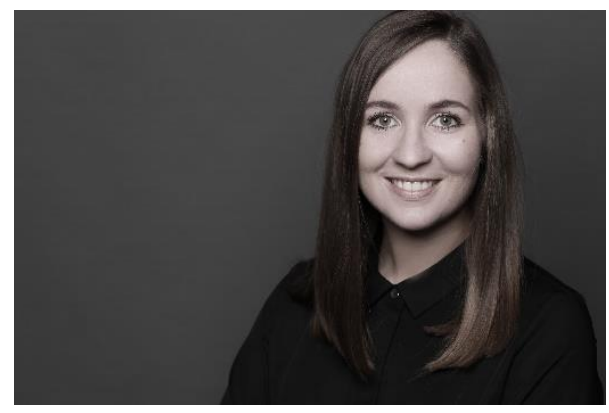
University, the Netherlands in 2009 and participated in the Erasmus programme, studying at Aarhus University, Denmark, in 2011. After obtaining the Bachelor of Science in 2012, she stayed at Maastricht University to study the Research Master of Cognitive and Clinical Neuroscience. Her master thesis internship on neurofeedback applications in Parkinson's Disease was supervised by Prof. Dr. David E.J. Linden at Cardiff University, UK, and Tabea obtained her Master of Science degree in 2014. In the same year, she started her PhD under the supervision of Prof. Dr. Rainer Goebel and Dr. Bettina Sorger at the Department of Cognitive Neuroscience at Maastricht University. Currently, she follows the training for psychological psychotherapists in Germany. 
A P PENDIX 


\section{PUBLICATIONS}

\section{PEER-REVIEWED JOURNALS}

Kamp, T., Sorger, B., Benjamins, C., Hausfeld, L., \& Goebel, R. (2018). The prestimulus default mode network state predicts cognitive task performance levels on a mental rotation task. Brain and Behavior, e01034. DOI: $10.1002 /$ brb3.1034

Kamp, T., Sorger, B., Lührs, M., Eck, J., \& Goebel, R. (submitted). Gradual selfregulation of the default mode network with real-time fMRI neurofeedback.

Haugg, A., Sladky, R., Skouras, S., McDonald, A., Craddock, C., Kirschner, M., Herdener, M., Koush, Y., Keynan, J., Hendler, T., Cohen Kadosh, K., Zich, C., Maclnnes, J., Adcock, A., Dickerson, K., Chen, N.-K., Young, K., Bodurka, J., Shuxia, Y., Becker, B., Auer, T., Schweizer, R., Pamplona, G., Emmert, K., Haller, S., van de Ville, D., Blefari, M.-L., Kim, D.-Y., Lee, J.-H., Marins, T., Megumi, F., Sorger, B., Kamp, T., Papoutsi, M., Liew, S.-L., Veit, R., Spetter, M., Weiskopf, N., \& Scharnowski, F. (submitted). Can we predict real-time fMRI neurofeedback learning success from pre-training brain activity?

Skottnik, L., Sorger, B., Kamp, T., Linden, D., \& Goebel, R. (submitted). Success and failure of controlling the real-time functional magnetic resonance imaging neurofeedback signal are reflected in the striatum.

Sorger, B.* Kamp, T.*, Weiskopf, N., Peters, J. C., \& Goebel, R. (2018). When the brain takes 'BOLD' steps: Real-time fMRI neurofeedback can further enhance the ability to gradually self-regulate regional brain activation. Neuroscience, 378, 71-88. DOI: 10.1016/j.neuroscience.2016.09.026

\footnotetext{
* Authors contributed equally and share first authorship
} 


\section{CONFERENCE CONTRIBUTIONS}

Kamp, T., Sorger, B., Lührs, M., \& Goebel, R. (2017). Localizing the default mode network for real-time purposes using seed-based analyses. Poster presented at the Real-time functional Imaging and Neurofeedback conference 2017 in Nara, Japan.

Kamp, T., Sorger, B., Benjamins, C., Hausfeld, L., \& Goebel, R. (2017). Is the prestimulus default mode network state predictive of cognitive task performance? Poster presented at Annual Meeting of the Organization for Human Brain Mapping 2017 in Vancouver, Canada.

Haugg, A., Sladky, R., Kirschner, M., Herdener, M., Koush, Y., Cohen Kadosh, K., Zich, C., Maclnnes, J., Adcock, A., Dickerson, K., Chen, N.-K., Young, K., Bodurka, J., Shuxia, Y., Becker, B., Auer, T., Schweizer, R., Emmert, K., Haller, S., van de Ville, D., Blefari, M.-L., Kim, D.-Y., Lee, J.-H., Marins, T., Megumi, F., Sorger, B., Kamp, T., Papoutsi, M., Liew, S.-L., Spetter, M., Veit, R., Weiskopf, N., \& Scharnowski, F. (2017). Pre-training localizer activity is related to real-time $\mathrm{fMRI}$ neurofeedback learning success. Poster presented at Annual Meeting of the Organization for Human Brain Mapping 2017 in Vancouver, Canada.

Skottnik, L., Sorger, B., Kamp, T., \& Goebel, R. (2017). Involvement of the reward system during rtfMRI neurofeedback across various self-regulation tasks. Poster presented at the Real-time functional Imaging and Neurofeedback conference 2017 in Nara, Japan. 
